\title{
A Regio- and Stereoselective Synthesis of Isoindolin-1-ones via Electrophilic Cyclization
}

\author{
Tuanli Yao and Richard C. Larock* \\ Department of Chemistry, Iowa State University, Ames, Iowa 50011
}

$\begin{array}{lc}\text { General } & \text { Page } \\ \text { Preparation of } o \text {-(1-alkynyl)benzamides } & \text { S2-S2 } \\ \text { Electrophilic cyclization of } o \text {-(1-alkynyl)benzamides by ICl } & \text { S2-S7 } \\ \text { Electrophilic cyclization of } o \text {-(1-alkynyl)benzamides by } \mathrm{I}_{2} & \mathrm{~S} 7-\mathrm{S} 9 \\ \text { Electrophilic cyclization of } o \text {-(1-alkynyl)benzamides by NBS } & \mathrm{S} 13-\mathrm{S} 13 \\ \text { Synthesis of cepharanone B } & \mathrm{S} 13-\mathrm{S} 15 \\ \text { References } & \mathrm{S} 16-\mathrm{S} 16 \\ \text { Copies of }{ }^{1} \mathrm{H} \text { and }{ }^{13} \mathrm{C} \text { NMR spectra } & \mathrm{S} 17-\mathrm{S} 74\end{array}$


General. The ${ }^{1} \mathrm{H}$ and ${ }^{13} \mathrm{C}$ NMR spectra were recorded at 300 and $75.5 \mathrm{MHz}$. Thinlayer chromatography was performed using commercially prepared 60-mesh silica gel plates, and visualization was effected with short wavelength UV light $(254 \mathrm{~nm})$. All melting points are uncorrected. All reagents were used directly as obtained commercially unless otherwise noted. The following starting materials were made according to literature procedures: 2 -iodobenzamide, ${ }^{1} \mathrm{~N}$-methyl-2-iodobenzamide, ${ }^{1} \mathrm{~N}$-benzyl-2iodobenzamide, ${ }^{1} \mathrm{~N}$-phenyl-2-iodobenzamide, ${ }^{1} \mathrm{~N}$ - $p$-anisyl-2-iodo-4,5dimethoxybenzamide, ${ }^{1}$ 3-iodoisonicotinanilide ${ }^{2}$ and ethyl 2-(phenylethynyl)cyclopent-1enecarboxylate. $^{3}$

General procedure for preparation of the $o$-(1-alkynyl)benzamides. To a solution of the corresponding organic iodide $(1.0 \mathrm{mmol})$ and the terminal alkyne $(1.2 \mathrm{mmol}, 1.2$ equiv) in $\mathrm{Et}_{3} \mathrm{~N}(4 \mathrm{ml})$ were added $\mathrm{PdCl}_{2}\left(\mathrm{PPh}_{3}\right)_{2}(1.4 \mathrm{mg}, 2 \mathrm{~mol} \%)$ and $\mathrm{CuI}(2.0 \mathrm{mg}, 1 \mathrm{~mol}$ $\%$ ). The resulting mixture was then heated under an $\mathrm{N}_{2}$ atm at $55^{\circ} \mathrm{C}$. The reaction was monitored by TLC to establish completion. When the reaction was complete, the mixture was allowed to cool to room temperature, and the ammonium salt was removed by filtration. The solvent was removed under reduced pressure and the residue was purified by column chromatography on silica gel to afford the corresponding $o$-(1alkynyl)benzamide.

N-Phenyl 2-(phenylethynyl)benzamide (1). Purification by flash chromatography (5:1 hexane/EtOAc) afforded $218 \mathrm{mg}(74 \%)$ of the product as a white solid with spectral properties identical to those previously reported: ${ }^{1} \mathrm{mp} 150-152{ }^{\circ} \mathrm{C}$ (lit. ${ }^{1} \mathrm{mp} 151-153{ }^{\circ} \mathrm{C}$ ). N-Methyl 2-(phenylethynyl)benzamide (10). Purification by flash chromatography (1:1 hexane/EtOAc) afforded $235 \mathrm{mg}(100 \%)$ of the product as a white solid with 
spectral properties identical to those previously reported: ${ }^{1} \mathrm{mp} 105-106{ }^{\circ} \mathrm{C}$ (lit. ${ }^{1} \mathrm{mp} 103$ $\left.105^{\circ} \mathrm{C}\right)$.

$N$-Benzyl 2-(phenylethynyl)benzamide (13). Purification by flash chromatography (3:1 hexane/EtOAc) afforded $280 \mathrm{mg}(90 \%)$ of the product as a white solid: $\mathrm{mp}$ 99-101 ${ }^{\circ} \mathrm{C} ;{ }^{1} \mathrm{H} \mathrm{NMR}\left(\mathrm{CDCl}_{3}\right) \delta 4.71(\mathrm{~d}, J=5.1 \mathrm{~Hz}, 2 \mathrm{H}), 7.11-7.15(\mathrm{~m}, 2 \mathrm{H}), 7.22-7.39(\mathrm{~m}, 8 \mathrm{H})$, 7.44-7.49 (m, 2H), 7.58-7.61 (m, 1H), 7.80 (s, 1H), 8.13-8.17 (m, 1H); ${ }^{13} \mathrm{C} \mathrm{NMR}\left(\mathrm{CDCl}_{3}\right)$ ઠ 44.8, 87.7, 96.0, 119.8, 127.8, 128.4, 128.6, 129.0, 129.1, 129.2, 130.5, 130.8, 131.6, 133.8, 135.3, 138.1, 166.3 (one carbon missing due to overlap); IR (neat, $\mathrm{cm}^{-1}$ ) 3310, 3061, 1636; HRMS Calcd for $\mathrm{C}_{22} \mathrm{H}_{17} \mathrm{NO}$ : 311.1310. Found: 311.1317 .

2-(Phenylethynyl)benzamide (16). Purification by flash chromatography (1:1 hexane/EtOAc) afforded $206 \mathrm{mg}(93 \%)$ of the product as a colorless solid with spectral properties identical to those previously reported: ${ }^{1} \mathrm{mp} 159-161{ }^{\circ} \mathrm{C}\left(\mathrm{lit} .{ }^{1} \mathrm{mp} 158-160{ }^{\circ} \mathrm{C}\right)$. $N$-Phenyl 2-(1-decynyl)benzamide (22). Purification by flash chromatography (7:1 hexane/EtOAc) afforded $200 \mathrm{mg}(60 \%)$ of the product as a colorless oil: ${ }^{1} \mathrm{H}$ NMR $\left(\mathrm{CDCl}_{3}\right) \delta 0.89(\mathrm{t}, J=6.7 \mathrm{~Hz}, 3 \mathrm{H}), 1.24-1.30(\mathrm{~m}, 8 \mathrm{H}), 1.38-1.44(\mathrm{~m}, 2 \mathrm{H}), 1.55-1.64(\mathrm{~m}$, 2H), $2.51(\mathrm{t}, J=7.2 \mathrm{~Hz}, 2 \mathrm{H}), 7.15(\mathrm{t}, J=7.5 \mathrm{~Hz}, 1 \mathrm{H}), 7.34-7.43(\mathrm{~m}, 4 \mathrm{H}), 7.49-7.53(\mathrm{~m}$, 1H), $7.68(\mathrm{~d}, J=7.5 \mathrm{~Hz}, 2 \mathrm{H}), 8.09-8.13(\mathrm{~m}, 1 \mathrm{H}), 9.42(\mathrm{~s}, 1 \mathrm{H}) ;{ }^{13} \mathrm{C} \mathrm{NMR}\left(\mathrm{CDCl}_{3}\right) \delta 14.4$, 20.0, 22.9, 28.8, 29.27, 29.32, 29.35, 32.1, 79.5, 98.8, 120.3, 120.5, 124.6, 128.6, 129.3, 130.4, 131.0, 134.0, 135.6, 138.4, 164.6; IR (neat, $\mathrm{cm}^{-1}$ ) 3345, 3061, 2956, 1673; HRMS Calcd for $\mathrm{C}_{23} \mathrm{H}_{27} \mathrm{NO}$ : 333.2093. Found: 333.2098 .

$N$-Phenyl 2-(trimethylsilylethynyl)benzamide (25). Purification by flash chromatography (4:1 hexane/EtOAc) afforded $142 \mathrm{mg}(48 \%)$ of the product as a 
colorless solid with spectral properties identical to those previously reported: ${ }^{1}$ mp 97-96 ${ }^{\circ} \mathrm{C}$ (lit. $\left.{ }^{1} \mathrm{mp} 95-96{ }^{\circ} \mathrm{C}\right)$.

$N$-Phenyl 2-(cyclohex-1-en-1-ylethynyl)benzamide (28). Purification by flash chromatography (4:1 hexane/EtOAc) afforded $263 \mathrm{mg}(88 \%)$ of the product as a yellow solid: $\mathrm{mp}$ 99-100 ${ }^{\circ} \mathrm{C} ;{ }^{1} \mathrm{H}$ NMR $\left(\mathrm{CDCl}_{3}\right) \delta$ 1.60-1.69 (m, 4H), 2.14-2.20 (m, 4H), 6.28 (q, $J=2.1 \mathrm{~Hz}, 1 \mathrm{H}), 7.14(\mathrm{t}, J=7.4 \mathrm{~Hz}, 1 \mathrm{H}), 7.33-7.44(\mathrm{~m}, 4 \mathrm{H}), 7.50-7.54(\mathrm{~m}, 1 \mathrm{H}), 7.70(\mathrm{~d}, J$ $=7.8 \mathrm{~Hz}, 2 \mathrm{H}), 8.10-8.14(\mathrm{~m}, 1 \mathrm{H}), 9.34(\mathrm{~s}, 1 \mathrm{H}) ;{ }^{13} \mathrm{C} \mathrm{NMR}\left(\mathrm{CDCl}_{3}\right) \delta$ 21.6, 22.4, 26.1, 29.2, 85.1, 99.1, 120.3, 120.3, 124.6, 128.8, 129.3, 130.6, 131.0, 133.7, 135.5, 137.7, 138.3, 164.5 (one carbon missing due to overlap); IR (neat, $\mathrm{cm}^{-1}$ ) 3308, 2930, 1671, 1653; HRMS Calcd for $\mathrm{C}_{21} \mathrm{H}_{19}$ NO: 301.1467 . Found: 301.1472.

$N$-Benzyl 4,5-dimethoxy-2-(trimethylsilylethynyl)benzamide (31). Purification by flash chromatography (7:1 hexane/EtOAc) afforded $332 \mathrm{mg}$ (90\%) of the product as a white solid: mp 95-96 ${ }^{\circ} \mathrm{C} ;{ }^{1} \mathrm{H}$ NMR $\left(\mathrm{CDCl}_{3}\right) \delta 0.09$ (s, 9H), 3.90 (s, 3H), $3.94(\mathrm{~s}, 3 \mathrm{H})$, $4.67(\mathrm{~d}, J=5.7 \mathrm{~Hz}, 2 \mathrm{H}), 6.93(\mathrm{~s}, 1 \mathrm{H}), 7.25-7.38(\mathrm{~m}, 5 \mathrm{H}), 7.77(\mathrm{~s}, 1 \mathrm{H}), 8.40(\mathrm{~s}, 1 \mathrm{H}),{ }^{13} \mathrm{C}$ NMR $\left(\mathrm{CDCl}_{3}\right) \delta$ 0.03, 44.6, 56.5, 56.6, 101.3, 104.4, 112.5, 113.3, 116.0, 127.9, 128.3, 128.8, 129.2, 138.7, 150.1, 150.9, 165.6; IR (neat, $\mathrm{cm}^{-1}$ ) 3386, 2959, 1654; HRMS Calcd for $\mathrm{C}_{21} \mathrm{H}_{25} \mathrm{NO}_{3}$ : 367.1604. Found: 367.1612 .

$N$-Phenyl 3-(phenylethynyl)isonicotinamide (37). Purification by flash chromatography (1:1 hexane/EtOAc) afforded $289 \mathrm{mg}(98 \%)$ of the product as a yellow solid: $\mathrm{mp} 149-150{ }^{\circ} \mathrm{C} ;{ }^{1} \mathrm{H}$ NMR $\left(\mathrm{CDCl}_{3}\right) \delta 7.19(\mathrm{t}, J=7.4 \mathrm{~Hz}, 1 \mathrm{H}), 7.30-7.44(\mathrm{~m}, 5 \mathrm{H})$, 7.51-7.55 (m, 2H), 7.63-7.67 (m, 2H), $7.96(\mathrm{~d}, J=5.1 \mathrm{~Hz}, 1 \mathrm{H}), 8.69(\mathrm{~d}, J=5.1 \mathrm{~Hz}, 1 \mathrm{H})$, $8.89(\mathrm{~s}, 1 \mathrm{H}), 9.34(\mathrm{~s}, 1 \mathrm{H}) ;{ }^{13} \mathrm{C} \mathrm{NMR}\left(\mathrm{CDCl}_{3}\right) \delta 84.5,99.8,115.8,120.4,121.3,123.3$, 
125.3, 129.0, 129.5, 130.1, 132.0, 137.6, 142.2, 149.9, 154.2, 162.4; IR (neat, $\left.\mathrm{cm}^{-1}\right) 3308$, 3041, 1661; HRMS Calcd for $\mathrm{C}_{20} \mathrm{H}_{14} \mathrm{~N}_{2} \mathrm{O}$ : 298.1106. Found: 298.1111 .

$N$-Phenyl-3-(1-heptynyl)isonicotinamide (40). Purification by flash chromatography (1:1 hexane/EtOAc) afforded $119 \mathrm{mg}(41 \%)$ of the product as a white solid: $\mathrm{mp}$ 91-92 ${ }^{\circ} \mathrm{C} ;{ }^{1} \mathrm{H}$ NMR $\left(\mathrm{CDCl}_{3}\right) \delta 0.87(\mathrm{t}, J=6.9 \mathrm{~Hz}, 3 \mathrm{H}), 1.26-1.45(\mathrm{~m}, 4 \mathrm{H}), 1.44-1.68(\mathrm{~m}, 2 \mathrm{H})$, $2.57(\mathrm{t}, J=7.2 \mathrm{~Hz}, 2 \mathrm{H}), 7.19(\mathrm{t}, J=7.5 \mathrm{~Hz}, 3 \mathrm{H}), 7.39(\mathrm{t}, J=8.4 \mathrm{~Hz}, 2 \mathrm{H}), 7.63-7.67(\mathrm{~m}$, 2H), $7.97(\mathrm{~d}, J=5.1 \mathrm{~Hz}, 1 \mathrm{H}), 8.65(\mathrm{~d}, J=5.4 \mathrm{~Hz}, 1 \mathrm{H}), 8.77(\mathrm{~s}, 1 \mathrm{H}), 9.46(\mathrm{~s}, 1 \mathrm{H}),{ }^{13} \mathrm{C}$ $\operatorname{NMR}\left(\mathrm{CDCl}_{3}\right) \delta 14.1,20.0,22.4,28.3,31.4,76.6,102.2,116.3,120.4,123.2,125.3$, 129.5, 137.6, 141.7, 149.3, 154.6, 162.3; IR (neat, $\mathrm{cm}^{-1}$ ) 3258, 3041, 1670; HRMS Calcd for $\mathrm{C}_{19} \mathrm{H}_{20} \mathrm{~N}_{2} \mathrm{O}$ : 292.1576. Found: 292.1579.

$N$-Phenyl-3-(cyclohex-1-en-1-ylethynyl)isonicotinamide (43). Purification by flash chromatography (1:1 hexane/EtOAc) afforded $269 \mathrm{mg}(89 \%)$ of the product as a white solid: $\mathrm{mp} 130-131{ }^{\circ} \mathrm{C} ;{ }^{1} \mathrm{H}$ NMR $\left(\mathrm{CDCl}_{3}\right) \delta$ 1.61-1.71 (m, 4H), 2.17-2.22 (m, 4H), 6.36 (q, $J=1.8 \mathrm{~Hz}, 1 \mathrm{H}), 7.18(\mathrm{t}, J=7.5 \mathrm{~Hz}, 1 \mathrm{H}), 7.38(\mathrm{t}, J=8.1 \mathrm{~Hz}, 2 \mathrm{H}), 7.67(\mathrm{~d}, J=7.5 \mathrm{~Hz}$, 2H), $7.97(\mathrm{~d}, J=5.1 \mathrm{~Hz}, 1 \mathrm{H}), 8.64(\mathrm{~d}, J=5.1 \mathrm{~Hz}, 1 \mathrm{H}), 8.77(\mathrm{~s}, 1 \mathrm{H}), 9.42(\mathrm{~s}, 1 \mathrm{H}) ;{ }^{13} \mathrm{C}$ $\operatorname{NMR}\left(\mathrm{CDCl}_{3}\right) \delta$ 21.5, 22.3, 26.1, 29.0, 82.1, 102.1, 116.3, 119.8, 120.4, 123.3, 125.2, 129.4, 137.6, 139.1, 141.4, 149.4, 154.2, 162.3; IR (neat, $\mathrm{cm}^{-1}$ ) 3302, 3046, 1656, 1599; HRMS Calcd for $\mathrm{C}_{20} \mathrm{H}_{18} \mathrm{~N}_{2} \mathrm{O}$ : 302.1419. Found: 302.1423 .

$N$-Phenyl-2-(phenylethynyl)cyclopent-1-ene-1-carboxamide (46). Ethyl 2(phenylethynyl)cyclopent-1-enecarboxylate (238 mg, $1.0 \mathrm{mmol}$ ) was dissolved in THF (4 $\mathrm{ml}$ ) and the resulting solution was added to an aqueous $1.0 \mathrm{M} \mathrm{LiOH}$ solution (3.0 ml, 3.0 mmol). The resulting mixture was stirred at $55^{\circ} \mathrm{C}$ for $24 \mathrm{~h}$ and then concentrated under reduced pressure. The residue was diluted with water $(10 \mathrm{ml})$ and washed with $\mathrm{Et}_{2} \mathrm{O}(3 \times$ 
$10 \mathrm{ml}$ ). The aqueous phase was cooled to $0{ }^{\circ} \mathrm{C}$, acidified with cold $10 \% \mathrm{H}_{2} \mathrm{SO}_{4}$ and extracted with $\mathrm{Et}_{2} \mathrm{O}(3 \times 25 \mathrm{ml})$. The organic extract was dried over $\mathrm{MgSO}_{4}$ and concentrated under reduced pressure to give the corresponding acid as a colorless solid.

The acid was dissolved in $(\mathrm{COCl})_{2}(5 \mathrm{ml})$ and stirred at room temperature overnight. The excess $(\mathrm{COCl})_{2}$ was removed under reduced pressure and the residue was diluted with THF ( $2 \mathrm{ml})$. The resulting solution was added slowly to a mixture of aniline (1.0 equiv) and pyridine ( 1 equiv) in THF $(2 \mathrm{ml})$ at $0{ }^{\circ} \mathrm{C}$. The reaction mixture was stirred at room temperature for $1 \mathrm{~h}$ and diluted with $\mathrm{Et}_{2} \mathrm{O}(20 \mathrm{ml})$, washed with $5 \% \mathrm{HCl}(8 \mathrm{ml})$ and $10 \% \mathrm{Na}_{2} \mathrm{CO}_{3}(8 \mathrm{ml})$, dried $\left(\mathrm{MgSO}_{4}\right)$ and filtered. The solvent was evaporated under reduced pressure and the product was isolated by flash chromatography (5:1 hexane/EtOAc) to afford $193 \mathrm{mg}$ (67\%) of the product as a yellow solid: $\mathrm{mp}$ 132-133 ${ }^{\circ} \mathrm{C} ;{ }^{1} \mathrm{H}$ NMR $\left(\mathrm{CDCl}_{3}\right) \delta$ 1.92-2.03 (m, 2H), 2.82-2.94 (m, 4H), $7.07(\mathrm{t}, J=7.5 \mathrm{~Hz}, 1 \mathrm{H})$, 7.28-7.43 (m, 5H), 7.50-7.54 (m, 2H), 7.61 (d, $J=7.8 \mathrm{~Hz}, 2 \mathrm{H}), 9.16(\mathrm{~s}, 1 \mathrm{H}) ;{ }^{13} \mathrm{C}$ NMR $\left(\mathrm{CDCl}_{3}\right) \delta 22.0,34.1,39.8,85.1,101.3,119.7,121.9,124.3,126.7,129.0,129.3,129.8$, 131.8, 138.4, 144.0, 162.5; IR (neat, $\mathrm{cm}^{-1}$ ) 3368, 3057, 1666, 1597; HRMS Calcd for $\mathrm{C}_{20} \mathrm{H}_{17} \mathrm{NO}: 287.1310$. Found: 287.1316 .

(3E)-3-(1,3-Diphenylprop-2-ynylidene)-2-phenylisoindolin-1-one (49). Purification by flash chromatography (7:1 hexane/EtOAc) afforded $343 \mathrm{mg}(86 \%)$ of the product as a yellow solid: mp $129-131{ }^{\circ} \mathrm{C} ;{ }^{1} \mathrm{H}$ NMR $\left(\mathrm{CDCl}_{3}\right) \delta 7.26(\mathrm{t}, J=10.5 \mathrm{~Hz}, 1 \mathrm{H}), 7.31-7.49$ (m, 10H), 7.57-7.72 (m, 4H), 7.94-7.98 (m, 2H), $8.08(\mathrm{~d}, J=7.5 \mathrm{~Hz}, 1 \mathrm{H}), 8.66(\mathrm{~d}, J=7.8$ $\mathrm{Hz}, 1 \mathrm{H}) ;{ }^{13} \mathrm{C} \mathrm{NMR}\left(\mathrm{CDCl}_{3}\right) \delta 87.7,97.3,102.0,123.4,123.9,124.3,124.4,125.4,128.3$, $128.4,128.8,128.9,129.0,129.4,130.5,130.9,131.8,132.7,135.3,136.4,145.6,152.2$, 
152.9; IR (neat, $\mathrm{cm}^{-1}$ ) 3056, 1689, 1588; HRMS Calcd for $\mathrm{C}_{29} \mathrm{H}_{19} \mathrm{NO}$ : 397.1467. Found: 397.1472.

General procedure for electrophilic cyclization of the alkynylarenecarboxamides

by ICl. The alkynylarenecarboxamide $(0.30 \mathrm{mmol})$ in $3 \mathrm{ml}$ of $\mathrm{CH}_{2} \mathrm{Cl}_{2}$ was placed in a 2 dram vial and flushed with $\mathrm{N}_{2}$. The $\mathrm{ICl}$ (1.2 equiv) in $0.5 \mathrm{ml}$ of $\mathrm{CH}_{2} \mathrm{Cl}_{2}$ was added dropwise to the vial by a syringe. The reaction was stirred at room temperature for 30 min unless otherwise indicated. The reaction mixture was then diluted with ether (50 $\mathrm{ml})$, washed with satd aq $\mathrm{Na}_{2} \mathrm{~S}_{2} \mathrm{O}_{3}(25 \mathrm{ml})$, dried $\left(\mathrm{MgSO}_{4}\right)$ and filtered. The solvent was evaporated under reduced pressure and the product was isolated by chromatography on a silica gel column.

4-Iodo-2,3-diphenylisoquinolin-1(2H)-one (3). Purification by flash chromatography (10:1 hexane/EtOAc) afforded $50.5 \mathrm{mg}(40 \%)$ of the product as a light yellow solid: $\mathrm{mp}$ 131-132 ${ }^{\circ} \mathrm{C} ;{ }^{1} \mathrm{H}$ NMR $\left(\mathrm{CDCl}_{3}\right) \delta 7.06(\mathrm{t}, J=7.3 \mathrm{~Hz}, 1 \mathrm{H}), 7.20-7.33(\mathrm{~m}, 4 \mathrm{H}), 7.39-7.41$ $(\mathrm{m}, 3 \mathrm{H}), 7.49(\mathrm{t}, J=7.5 \mathrm{~Hz}, 1 \mathrm{H}), 7.59-7.67(\mathrm{~m}, 3 \mathrm{H}), 7.76(\mathrm{~d}, J=7.5 \mathrm{~Hz}, 1 \mathrm{H}), 8.40(\mathrm{~d}, J=$ $7.8 \mathrm{~Hz}, 1 \mathrm{H}) ;{ }^{13} \mathrm{C} \mathrm{NMR}\left(\mathrm{CDCl}_{3}\right) \delta 123.1,124.0,124.1,127.7,128.2,128.9,129.4,130.1$, 130.2, 131.5, 133.2, 135.0, 135.6, 146.1, 148.7, 153.4; IR (neat, $\mathrm{cm}^{-1}$ ) 1645; HRMS Calcd for $\mathrm{C}_{21} \mathrm{H}_{14} \mathrm{INO}$ : 423.0120. Found: 423.0129 .

2-Benzyl-4-iodo-3-phenylisoquinolin-1(2H)-one (15). Purification by flash chromatography (7:1 hexane/EtOAc) afforded $64 \mathrm{mg}(30 \%)$ of the product as a yellow oil: ${ }^{1} \mathrm{H}$ NMR $\left(\mathrm{CDCl}_{3}\right) \delta 4.70(\mathrm{~s}, 2 \mathrm{H}), 7.25-7.28(\mathrm{~m}, 1 \mathrm{H})$, 7.32-7.50 (m, 8H), 7.58-7.72 $(\mathrm{m}, 4 \mathrm{H}), 8.32(\mathrm{dd}, J=1.2,7.8 \mathrm{~Hz}, 1 \mathrm{H}) ;{ }^{13} \mathrm{C} \mathrm{NMR}\left(\mathrm{CDCl}_{3}\right) \delta 50.4,75.8,124.1,126.7$, $127.0,127.9,128.3,128.5,129.2,130.0,130.1,131.3,132.6,134.1,136.3,141.0,150.1$, 
153.6; IR (neat, $\mathrm{cm}^{-1}$ ) 3061, 2923, 1663, 1601; HRMS Calcd for $\mathrm{C}_{22} \mathrm{H}_{16} \mathrm{INO}$ : 437.0277 . Found: 437.0281.

3-(Cyclohex-1-en-1-yl)-4-iodo-2-phenylisoquinolin-1(2H)-one (30). Purification by flash chromatography (7:1 hexane/EtOAc) afforded $94 \mathrm{mg}(74 \%)$ of the product as a yellow oil: ${ }^{1} \mathrm{H}$ NMR $\left(\mathrm{CDCl}_{3}\right) \delta$ 1.60-1.70 (m, 4H), 2.14-2.21 (m, 4H), 6.14 (q, $J=1.8$, $1 \mathrm{H}), 7.12(\mathrm{tt}, J=1.2,7.5 \mathrm{~Hz}, 1 \mathrm{H}), 7.19(\mathrm{dd}, J=1.2,7.5 \mathrm{~Hz}, 2 \mathrm{H}), 7.31-7.45(\mathrm{~m}, 3 \mathrm{H}), 7.59$ $(\mathrm{dt}, J=1.2,7.8 \mathrm{~Hz}, 1 \mathrm{H}), 7.67(\mathrm{dd}, J=0.6,8.1 \mathrm{~Hz}, 1 \mathrm{H}), 8.33(\mathrm{dd}, J=0.9,7.8 \mathrm{~Hz}, 1 \mathrm{H}) ;{ }^{13} \mathrm{C}$ $\operatorname{NMR}\left(\mathrm{CDCl}_{3}\right) \delta$ 21.7, 22.5, 25.2, 26.6, 74.1, 123.1, 124.0, 127.5, 128.8, 128.9, 131.5, 133.1, 133.9, 135.1, 135.3, 146.3, 149.1, 155.8 (one carbon missing due to overlap); IR (neat, $\mathrm{cm}^{-1}$ ) 3063, 2933, 1652, 1592; HRMS Calcd for $\mathrm{C}_{21} \mathrm{H}_{18} \mathrm{INO}$ : 427.0433. Found: 427.0439.

4-Iodo-2,3-diphenyl-2,6-naphthyridin-1(2H)-one (39). Purification by flash chromatography (4:1 hexane/EtOAc) afforded $45 \mathrm{mg}(53 \%)$ of the product as a yellow solid: $\mathrm{mp} 164-165^{\circ} \mathrm{C} ;{ }^{1} \mathrm{H}$ NMR $\left(\mathrm{CDCl}_{3}\right) \delta 7.12$ (tt, $\left.J=1.5,7.5 \mathrm{~Hz}, 1 \mathrm{H}\right), 7.22-7.34(\mathrm{~m}$, $4 \mathrm{H}), 7.39-7.46(\mathrm{~m}, 3 \mathrm{H}), 7.59-7.63(\mathrm{~m}, 2 \mathrm{H}), 8.11(\mathrm{~s}, 1 \mathrm{H}), 8.77(\mathrm{~s}, 1 \mathrm{H}), 9.08(\mathrm{~s}, 1 \mathrm{H}) ;{ }^{13} \mathrm{C}$ NMR $\left(\mathrm{CDCl}_{3}\right) \delta 70.3,123.3,125.1,128.3,129.0,130.1,130.2,130.5,130.9,134.8$, 145.0, 146.6, 150.0, 153.7, 154.6 (one carbon missing due to overlap); IR (neat, $\mathrm{cm}^{-1}$ ) 3061, 2930, 1669, 1651; HRMS Calcd for $\mathrm{C}_{20} \mathrm{H}_{13} \mathrm{IN}_{2} \mathrm{O}$ : 424.0073. Found: 424.0081.

4-Iodo-3-pentyl-2-phenyl-2,6-naphthyridin-1(2H)-one (42). Purification by flash chromatography (4:1 hexane/EtOAc) afforded $51 \mathrm{mg}(40 \%)$ of the product as a yellow oil: ${ }^{1} \mathrm{H}$ NMR $\left(\mathrm{CDCl}_{3}\right) \delta$ 0.84-0.89 (m, 3H), 1.27-1.33 (m, 4H), 1.55-1.61 (m, 2H), 2.74 (t, $J=7.5 \mathrm{~Hz}, 2 \mathrm{H}), 7.11-7.20(\mathrm{~m}, 3 \mathrm{H}), 7.36(\mathrm{dt}, J=1.8,7.5 \mathrm{~Hz}, 2 \mathrm{H}), 8.00(\mathrm{~d}, J=5.1 \mathrm{~Hz}$, $1 \mathrm{H}), 8.66(\mathrm{~d}, J=5.1 \mathrm{~Hz}, 1 \mathrm{H}), 8.87(\mathrm{~s}, 1 \mathrm{H}) ;{ }^{13} \mathrm{C} \mathrm{NMR}\left(\mathrm{CDCl}_{3}\right) \delta 14.1,22.5,26.8,31.2$, 
$36.6,70.1,119.0,122.9,124.8,128.5,129.0,130.4,145.4,147.1,149.5,152.5,158.1 ;$ IR (neat, $\mathrm{cm}^{-1}$ ) 2956, 2928, 1661, 1608; HRMS Calcd for $\mathrm{C}_{19} \mathrm{H}_{19} \mathrm{IN}_{2} \mathrm{O}$ : 418.0542. Found: 418.0551.

\section{3-(Cyclohex-1-en-1-yl)-4-iodo-2-phenyl-2,6-naphthyridin-1(2H)-one (45).}

Purification by flash chromatography (7:1 hexane/EtOAc) afforded $51 \mathrm{mg}(60 \%)$ of the product as a yellow solid: $\mathrm{mp} 111-112{ }^{\circ} \mathrm{C} ;{ }^{1} \mathrm{H}$ NMR $\left(\mathrm{CDCl}_{3}\right) \delta 1.60-1.71(\mathrm{~m}, 4 \mathrm{H}), 2.11$ $2.21(\mathrm{~m}, 4 \mathrm{H}), 6.18(\mathrm{q}, J=2.1 \mathrm{~Hz}, 1 \mathrm{H}), 7.13(\mathrm{tt}, J=1.2,7.5 \mathrm{~Hz}, 1 \mathrm{H}), 7.19-7.23(\mathrm{~m}, 2 \mathrm{H})$, 7.32-7.38 (m, 2H), $8.02(\mathrm{dd}, J=0.6,5.1 \mathrm{~Hz}, 1 \mathrm{H}), 8.66(\mathrm{~d}, J=5.1 \mathrm{~Hz}, 1 \mathrm{H}), 8.95(\mathrm{~s}, 1 \mathrm{H})$;

${ }^{13} \mathrm{C}$ NMR $\left(\mathrm{CDCl}_{3}\right) \delta$ 21.7, 22.4, 25.2, 26.4, 68.4, 119.0, 123.2, 124.9, 128.9, 129.1, 130.7, 133.2, 136.2, 145.2, 147.0, 149.6, 153.7, 157.1; IR $\left(\right.$ neat, $\left.\mathrm{cm}^{-1}\right)$ 2930, 1652, 1592; HRMS Calcd for $\mathrm{C}_{20} \mathrm{H}_{17} \mathrm{IN}_{2} \mathrm{O}$ : 428.0386. Found: 428.0393 .

General procedure for electrophilic cyclization of the alkynylarenecarboxamides

by $\mathbf{I}_{2}$. The alkynylarenecarboxamide $(0.30 \mathrm{mmol}), \mathrm{I}_{2}$ ( 3.0 equiv), $\mathrm{NaHCO}_{3}$ ( 3.0 equiv) and $\mathrm{CH}_{3} \mathrm{CN}(3 \mathrm{ml})$ were placed in a 4 dram vial and flushed with $\mathrm{N}_{2}$. The reaction mixture was stirred at room temperature for $1 \mathrm{~h}$ unless otherwise indicated. The reaction mixture was then diluted with ether $(50 \mathrm{ml})$, washed with satd aq $\mathrm{Na}_{2} \mathrm{~S}_{2} \mathrm{O}_{3}(25 \mathrm{ml})$, dried $\left(\mathrm{MgSO}_{4}\right)$ and filtered. The solvent was evaporated under reduced pressure and the product was isolated by chromatography on a silica gel column.

(3E)-3-[Iodo(phenyl)methylene]-2-phenylisoindolin-1-one (2). Purification by flash chromatography (7:1 hexane/EtOAc) afforded $110 \mathrm{mg}(86 \%)$ of the product as a white solid: $\mathrm{mp} 97-99{ }^{\circ} \mathrm{C} ;{ }^{1} \mathrm{H} \mathrm{NMR}\left(\mathrm{CDCl}_{3}\right) \delta 7.09(\mathrm{t}, J=6.6 \mathrm{~Hz}, 1 \mathrm{H}), 7.22-7.36(\mathrm{~m}, 7 \mathrm{H})$, 7.59-7.73 (m, 4H), $8.05(\mathrm{~d}, J=7.5 \mathrm{~Hz}, 1 \mathrm{H}), 8.86(\mathrm{~d}, J=7.8 \mathrm{~Hz}, 1 \mathrm{H}) ;{ }^{13} \mathrm{C} \mathrm{NMR}\left(\mathrm{CDCl}_{3}\right) \delta$ 124.1, 125.07, 125.1, 125.4, 128.1, 128.7, 130.5, 130.9, 132.0, 132.8, 135.8, 140.6, 145.0, 
147.8, 152.0 (one carbon missing due to overlap); IR (neat, $\mathrm{cm}^{-1}$ ) 1684; HRMS Calcd for $\mathrm{C}_{21} \mathrm{H}_{14}$ INO: 423.0120. Found: 423.0129.

(3E)-3-[Iodo(phenyl)methylene]-2-methylisoindolin-1-one (11). Purification by flash chromatography (7:1 hexane/EtOAc) afforded $86 \mathrm{mg}(80 \%)$ of the product as a yellow solid: $\operatorname{mp~} 122-125{ }^{\circ} \mathrm{C} ;{ }^{1} \mathrm{H}$ NMR $\left(\mathrm{CDCl}_{3}\right) \delta 3.16(\mathrm{~s}, 3 \mathrm{H}), 7.28(\mathrm{t}, J=7.2 \mathrm{~Hz}, 1 \mathrm{H})$, $7.39(\mathrm{t}, J=7.5 \mathrm{~Hz}, 2 \mathrm{H}), 7.52-7.67(\mathrm{~m}, 4 \mathrm{H}), 7.85(\mathrm{~d}, J=7.5 \mathrm{~Hz}, 1 \mathrm{H}), 8.83(\mathrm{~d}, J=7.8 \mathrm{~Hz}$ $1 \mathrm{H}) ;{ }^{13} \mathrm{C} \mathrm{NMR}\left(\mathrm{CDCl}_{3}\right) \delta 35.2,73.5,123.2,125.2,128.2,128.5,130.4,130.8,131.4$, 132.0, 136.2, 140.9, 147.5, 154.9; IR (neat, $\mathrm{cm}^{-1}$ ) 1714; HRMS Calcd for $\mathrm{C}_{16} \mathrm{H}_{12} \mathrm{INO}$ : 360.9964. Found: 360.9968.

(3E)-2-Benzyl-3-[iodo(phenyl)methylene]isoindolin-1-one (14). Purification by flash chromatography (7:1 hexane/EtOAc) afforded $107 \mathrm{mg}(85 \%)$ of the product as a yellow solid: $\operatorname{mp~} 70-72{ }^{\circ} \mathrm{C} ;{ }^{1} \mathrm{H}$ NMR $\left(\mathrm{CDCl}_{3}\right) \delta 4.64(\mathrm{~s}, 2 \mathrm{H}), 7.23-7.34(\mathrm{~m}, 6 \mathrm{H}), 7.41(\mathrm{t}, J$ $=7.5 \mathrm{~Hz}, 2 \mathrm{H}), 7.55-7.66(\mathrm{~m}, 4 \mathrm{H}), 7.95(\mathrm{~d}, J=7.5 \mathrm{~Hz}, 1 \mathrm{H}), 8.83(\mathrm{~d}, J=8.1 \mathrm{~Hz}, 1 \mathrm{H}) ;{ }^{13} \mathrm{C}$ $\operatorname{NMR}\left(\mathrm{CDCl}_{3}\right) \delta 52.1,73.7,123.6,125.2,126.9,128.2,128.3,128.58,128.59,130.4$, 130.8, 131.6, 132.1, 136.2, 140.2, 141.0, 147.5, 154.5; IR (neat, $\mathrm{cm}^{-1}$ ) 3058, 3027, 1694, 1612; HRMS Calcd for $\mathrm{C}_{22} \mathrm{H}_{16} \mathrm{INO}$ : 437.0277. Found: 437.0283.

(3E)-3-(1-Iodononylidene)-2-phenylisoindolin-1-one (23). Purification by flash chromatography (12:1 hexane/EtOAc) afforded $123 \mathrm{mg}(90 \%)$ of the product as a yellow liquid: ${ }^{1} \mathrm{H} \mathrm{NMR}\left(\mathrm{CDCl}_{3}\right) \delta$ 0.87-0.92 (m, 3H), 1.22-1.32 (m, 10H), 1.59-1.65 (m, 2H), $2.90(\mathrm{t}, J=7.5,2 \mathrm{H}), 7.15-7.21(\mathrm{~m}, 1 \mathrm{H}), 7.35-7.46(\mathrm{~m}, 4 \mathrm{H}), 7.55-7.66(\mathrm{~m}, 2 \mathrm{H}), 8.02(\mathrm{dd}, J$ $=0.9,7.2 \mathrm{~Hz}, 1 \mathrm{H}), 8.69(\mathrm{dd}, J=0.9,7.8 \mathrm{~Hz}, 1 \mathrm{H}) ;{ }^{13} \mathrm{C} \mathrm{NMR}\left(\mathrm{CDCl}_{3}\right) \delta 14.4,22.9,28.7$, 29.3, 29.5, 29.6, 32.1, 39.9, 82.8, 124.0, 124.3, 124.4, 125.0, 128.9, 130.3, 131.9, 132.6, 
135.7, 145.8, 147.4, 152.6; IR (neat, $\mathrm{cm}^{-1}$ ) 3056, 2924, 1693, 1590; HRMS Calcd for $\mathrm{C}_{23} \mathrm{H}_{26} \mathrm{IN}_{2} \mathrm{O}$ : 459.1059. Found: 459.1068.

(3E)-3-[Iodo(trimethylsilyl)methylene]-2-phenylisoindolin-1-one (26). Purification by flash chromatography (7:1 hexane/EtOAc) afforded $95 \mathrm{mg}(77 \%)$ of the product as a yellow solid: $\mathrm{mp} 91-92{ }^{\circ} \mathrm{C} ;{ }^{1} \mathrm{H}$ NMR $\left(\mathrm{CDCl}_{3}\right) \delta 0.18(\mathrm{t}, J=3.6 \mathrm{~Hz}, 9 \mathrm{H}), 7.10-7.20(\mathrm{~m}$, $3 \mathrm{H}), 7.35(\mathrm{t}, J=7.5 \mathrm{~Hz}, 2 \mathrm{H}), 7.60-7.67(\mathrm{~m}, 2 \mathrm{H}), 8.02(\mathrm{~d}, J=8.1 \mathrm{~Hz}, 1 \mathrm{H}), 8.96(\mathrm{td}, J=$ $0.9,8.1 \mathrm{~Hz}, 1 \mathrm{H}) ;{ }^{13} \mathrm{C} \mathrm{NMR}\left(\mathrm{CDCl}_{3}\right) \delta 0.0,80.2,122.3,123.4,123.9,125.4,128.3,130.4$, 131.5, 132.0, 136.2, 146.1, 152.8, 154.8; IR (neat, $\mathrm{cm}^{-1}$ ) 1694, 1591, 1486; HRMS Calcd for CHINO: 419.0202. Found: 419.0213.

(3E)-3-[Cyclohex-1-en-1-yl(iodo)methylene]-2-phenylisoindolin-1-one (29).

Purification by flash chromatography (7:1 hexane/EtOAc) afforded $41 \mathrm{mg}(31 \%)$ of the product as a yellow liquid: ${ }^{1} \mathrm{H}$ NMR $\left(\mathrm{CDCl}_{3}\right) \delta$ 1.71-1.89 (m, 5H), 2.14-2.34 (m, 5H), $6.11(\mathrm{q}, J=1.8 \mathrm{~Hz}, 1 \mathrm{H}), 7.20(\mathrm{t}, J=7.5 \mathrm{~Hz}, 1 \mathrm{H}), 7.41(\mathrm{t}, J=8.1 \mathrm{~Hz}, 2 \mathrm{H}), 7.48-7.53(\mathrm{~m}$, 2H), 7.66 (dd, $J=0.9,8.1 \mathrm{~Hz}, 2 \mathrm{H}), 7.76-7.80(\mathrm{~m}, 1 \mathrm{H}), 7.94-7.98(\mathrm{~m}, 1 \mathrm{H}) ;{ }^{13} \mathrm{C} \mathrm{NMR}$ $\left(\mathrm{CDCl}_{3}\right) \delta$ 22.1, 22.8, 26.1, 28.0, 81.8, 122.7, 124.1, 125.3, 125.6, 129.0, 129.9, 130.9, 132.2, 132.9, 133.9, 136.8, 145.0, 149.8, 152.2; IR (neat, $\mathrm{cm}^{-1}$ ) 2928, 1690, 1591; HRMS Calcd for $\mathrm{C}_{21} \mathrm{H}_{18} \mathrm{INO}: 427.0433$. Found: 427.0439 .

(3E)- 2-Benzyl 3-[iodo(trimethylsilyl)methylene]-5,6-dimethoxyisoindolin-1-one (32). Purification by flash chromatography (2:1 hexane/EtOAc) afforded $119 \mathrm{mg}(80 \%)$ of the product as a colorless oil: ${ }^{1} \mathrm{H}$ NMR $\left(\mathrm{CDCl}_{3}\right) \delta 0.37(\mathrm{~s}, 9 \mathrm{H}), 3.94(\mathrm{~s}, 3 \mathrm{H}), 3.98(\mathrm{~s}$, 3H), $4.78(\mathrm{~s}, 2 \mathrm{H}), 7.24(\mathrm{t}, J=3.0 \mathrm{~Hz}, 1 \mathrm{H}), 7.29-7.31(\mathrm{~m}, 1 \mathrm{H}), 7.32-7.35(\mathrm{~m}, 2 \mathrm{H}), 7.38-$ $7.41(\mathrm{~m}, 2 \mathrm{H}), 8.41(\mathrm{~s}, 1 \mathrm{H}) ;{ }^{13} \mathrm{C} \mathrm{NMR}\left(\mathrm{CDCl}_{3}\right) \delta$ 0.7, 52.2, 56.4, 56.5, 76.8, 104.1, 107.5, 
$125.8,126.9,127.8,128.5,130.3,140.1,151.82,151.85,154.5,155.2 ;$ IR (neat, $\mathrm{cm}^{-1}$ ) 2956, 1699, 1589, 1495; HRMS Calcd for $\mathrm{C}_{21} \mathrm{H}_{24} \mathrm{INO}_{3}$ : 493.0570. Found: 493.0580 . (3E)-3-[Iodo(phenyl)methylene]-2-phenyl-2,3-dihydro-1H-pyrrolo[3,4-c]pyridin1-one (38). Purification by flash chromatography (4:1 hexane/EtOAc) afforded $79 \mathrm{mg}$ (63\%) of the product as a yellow solid: $\mathrm{mp} 165-167{ }^{\circ} \mathrm{C}$ (decompose); ${ }^{1} \mathrm{H}$ NMR $\left(\mathrm{CDCl}_{3}\right)$ $\delta 7.14(\mathrm{t}, J=5.4 \mathrm{~Hz}, 1 \mathrm{H}), 7.23-7.41(\mathrm{~m}, 7 \mathrm{H}), 7.59-7.63(\mathrm{~m}, 2 \mathrm{H}), 7.90(\mathrm{~d}, J=4.5 \mathrm{~Hz}, 1 \mathrm{H})$, $8.86(\mathrm{~s}, 1 \mathrm{H}), 10.0(\mathrm{~s}, 1 \mathrm{H}) ;{ }^{13} \mathrm{C} \mathrm{NMR}\left(\mathrm{CDCl}_{3}\right) \delta$ 77.8, 117.2, 125.4, 126.4, 128.2, 128.9, 129.1, 130.4, 139.9, 140.0, 144.1, 147.0, 147.1, 150.2, 150.7; IR (neat, $\mathrm{cm}^{-1}$ ) 3020, 1687, 1424; HRMS Calcd for $\mathrm{C}_{20} \mathrm{H}_{13} \mathrm{IN}_{2} \mathrm{O}$ : 424.0073. Found: 424.0081.

\section{(3E)-3-(1-Iodohexylidene)-2-phenyl-2,3-dihydro-1H-pyrrolo[3,4-c]pyridin-1-one}

(41). Purification by flash chromatography (4:1 hexane/EtOAc) afforded $68.4 \mathrm{mg}(55 \%)$

of the product as a yellow solid: $\mathrm{mp} 100-101{ }^{\circ} \mathrm{C} ;{ }^{1} \mathrm{H}$ NMR $\left(\mathrm{CDCl}_{3}\right) \delta 0.86-0.91(\mathrm{t}, J=6.9$ $\mathrm{Hz}, 3 \mathrm{H}), 1.30-1.35(\mathrm{~m}, 4 \mathrm{H}), 1.60-1.66(\mathrm{~m}, 2 \mathrm{H}), 2.90(\mathrm{t}, J=7.5 \mathrm{~Hz}, 2 \mathrm{H}), 7.20(\mathrm{t}, J=6.9$ $\mathrm{Hz}, 1 \mathrm{H}), 7.36-7.46(\mathrm{~m}, 4 \mathrm{H}), 7.86(\mathrm{~d}, J=5.1 \mathrm{~Hz}, 1 \mathrm{H}), 8.81(\mathrm{~d}, J=5.1 \mathrm{~Hz}, 1 \mathrm{H}), 10.0(\mathrm{~s}$, $1 \mathrm{H}) ;{ }^{13} \mathrm{C} \mathrm{NMR}\left(\mathrm{CDCl}_{3}\right) \delta$ 14.2, 22.6, 28.9, 30.8, 39.7, 85.2, 117.1, 124.6, 125.9, 129.0, 130.9, 139.9, 144.8, 146.4, 146.7, 150.2, 150.8; IR (neat, $\left.\mathrm{cm}^{-1}\right)$ 2980, 1683, 1422; HRMS Calcd for $\mathrm{C}_{19} \mathrm{H}_{19} \mathrm{IN}_{2} \mathrm{O}$ : 418.0542. Found: 418.0552 .

\section{4-Iodo-2,3-diphenyl-2,5,6,7-tetrahydro-1H-cyclopenta[c]pyridin-1-one (48) .}

Purification by flash chromatography (5:1 hexane/EtOAc) afforded $71 \mathrm{mg}(58 \%)$ of the product as a yellow solid: $\mathrm{mp} 148-150{ }^{\circ} \mathrm{C} ;{ }^{1} \mathrm{H}$ NMR $\left(\mathrm{CDCl}_{3}\right) \delta 2.12(\mathrm{q}, J=7.5 \mathrm{~Hz}, 2 \mathrm{H})$, 2.85-2.92 (m, 2H), 3.02-3.08 (m, 2H), $7.01(\mathrm{td}, J=1.2,7.2 \mathrm{~Hz}, 1 \mathrm{H}), 7.15(\mathrm{dd}, J=1.2,8.4$ $\mathrm{Hz}, 2 \mathrm{H}), 7.26(\mathrm{dt}, J=1.8,6.9 \mathrm{~Hz}, 2 \mathrm{H}), 7.33-7.39(\mathrm{~m}, 3 \mathrm{H}), 7.57-7.62(\mathrm{~m}, 2 \mathrm{H}) ;{ }^{13} \mathrm{C} \mathrm{NMR}$ $\left(\mathrm{CDCl}_{3}\right) \delta 21.7,32.9,40.3,69.7,123.1,123.6,128.2,128.7,129.4,129.6,130.2,134.2$, 
146.4, 149.4, 152.9, 156.1; IR (neat, $\mathrm{cm}^{-1}$ ) 2954, 1650, 1635, 1487; HRMS Calcd for $\mathrm{C}_{20} \mathrm{H}_{16} \mathrm{INO}: 413.0277$. Found: 413.0285.

General procedure for electrophilic cyclization of the $o$-(1-alkynyl)benzamides by

NBS. The alkynylamide $(0.30 \mathrm{mmol}), \mathrm{NBS}\left(1.5\right.$ equiv) and $\mathrm{CH}_{2} \mathrm{Cl}_{2}(3 \mathrm{ml})$ were placed in a 2 dram vial and flushed with $\mathrm{N}_{2}$. The reaction mixture was stirred at room temperature for $1 \mathrm{~h}$ unless otherwise indicated. The solvent was evaporated under reduced pressure and the product was isolated by chromatography on a silica gel column.

(3E)-3-[Bromo(phenyl)methylene]-2-phenylisoindolin-1-one (4). Purification by flash chromatography (5:1 hexane/EtOAc) afforded $93.2 \mathrm{mg}(82 \%)$ of the product as a white solid: $\operatorname{mp} 90-92{ }^{\circ} \mathrm{C} ;{ }^{1} \mathrm{H}$ NMR $\left(\mathrm{CDCl}_{3}\right) \delta 7.13(\mathrm{t}, J=7.2 \mathrm{~Hz}, 1 \mathrm{H}), 7.26-7.41(\mathrm{~m}$, 7H), 7.60-7.75 (m, 4H), $8.06(\mathrm{~d}, J=6.6 \mathrm{~Hz}, 1 \mathrm{H}), 8.64(\mathrm{~d}, J=8.1 \mathrm{~Hz}, 1 \mathrm{H})$; IR (neat, $\left.\mathrm{cm}^{-1}\right)$ 1690; HRMS Calcd for $\mathrm{C}_{16} \mathrm{H}_{12} \mathrm{BrNO}$ : 375.0259 . Found: 375.0266 .

\section{Synthesis of cepharanone B.}

\section{2-Iodo-4,5-dimethoxy- $N$-(4-methoxybenzyl)benzamide (51). 2-Iodo-4,5-}

dimethoxybenzoic acid $(3.7 \mathrm{~g}, 12 \mathrm{mmol})$ and $\mathrm{SOCl}_{2}(18 \mathrm{ml})$ were refluxed at $80{ }^{\circ} \mathrm{C}$ for 0.5

h. Excess $\mathrm{SOCl}_{2}$ was removed under reduced pressure and a solution of pyridine (3 equiv) in THF (30 ml) was added to the residue. The resulting mixture was cooled to 0 ${ }^{\circ} \mathrm{C}$ and a solution of 4-methoxybenzylamine (1.0 equiv) in THF (10 ml ) was added dropwise. The resulting reaction mixture was stirred at $0{ }^{\circ} \mathrm{C}$ for $0.5 \mathrm{~h}$, followed by stirring at room temperature for $3 \mathrm{~h}$. The mixture was then diluted with $\mathrm{CHCl}_{3}(100 \mathrm{ml})$, washed with $2 \mathrm{~N} \mathrm{HCl}(25 \mathrm{ml})$ and satd $\mathrm{NaHCO}_{3}(25 \mathrm{ml})$, dried $\left(\mathrm{MgSO}_{4}\right)$ and filtered. The solvent was evaporated under reduced pressure and the product was isolated by flash chromatography (1:2 hexane/EtOAc) to afford $3.24 \mathrm{~g}(65 \%)$ of the product as white 
solid: $\mathrm{mp} 159-160{ }^{\circ} \mathrm{C} ;{ }^{1} \mathrm{H}$ NMR $\left(\mathrm{CDCl}_{3}\right) \delta$ 3.77-3.81 (m, 3H), 3.83-3.88 (m, 6H), 4.51$4.56(\mathrm{~m}, 2 \mathrm{H}), 6.23(\mathrm{~s}, 1 \mathrm{H}), 6.83-6.89(\mathrm{~m}, 2 \mathrm{H}), 6.96(\mathrm{~s}, 1 \mathrm{H}), 7.16(\mathrm{~s}, 1 \mathrm{H}), 7.29-7.32(\mathrm{~m}$, $2 \mathrm{H}) ;{ }^{13} \mathrm{C} \mathrm{NMR}\left(\mathrm{CDCl}_{3}\right) \delta 44.0,55.5,56.3,56.5,81.2,112.1,114.3,122.2,129.8,129.9$, 134.4, 149.3, 150.6, 159.3, 168.8; IR (neat, $\mathrm{cm}^{-1}$ ) 3300, 2964, 1638, 1498; HRMS Calcd for $\mathrm{C}_{17} \mathrm{H}_{18} \mathrm{INO}_{4}$ : 427.0281. Found: 427.0291.

\section{$N$-(4-Methoxybenzyl)-4,5-dimethoxy-2-[(trimethylsilyl)ethynyl]benzamide (52).}

This compound was prepared by following the general procedure for preparation of the $o$ (1-alkynyl)benzamides. Purification by flash chromatography (3:2 hexane/EtOAc) afforded $277 \mathrm{mg}(70 \%)$ of the product as a white solid: $\mathrm{mp} 107-108{ }^{\circ} \mathrm{C} ;{ }^{1} \mathrm{H} \mathrm{NMR}$ $\left(\mathrm{CDCl}_{3}\right) \delta 0.10(\mathrm{~s}, 9 \mathrm{H}), 3.79(\mathrm{~s}, 3 \mathrm{H}), 3.92(\mathrm{~s}, 3 \mathrm{H}), 3.95(\mathrm{~s}, 3 \mathrm{H}), 4.60(\mathrm{~d}, J=5.4 \mathrm{~Hz}, 2 \mathrm{H})$, $6.86(\mathrm{dd}, J=1.8,6.9 \mathrm{~Hz}, 2 \mathrm{H}), 6.93(\mathrm{~s}, 1 \mathrm{H}), 7.29(\mathrm{~d}, J=8.4 \mathrm{~Hz}, 2 \mathrm{H}), 7.77(\mathrm{~s}, 1 \mathrm{H}), 8.32(\mathrm{~s}$, $1 \mathrm{H}) ;{ }^{13} \mathrm{C} \mathrm{NMR}\left(\mathrm{CDCl}_{3}\right) \delta 0.0,44.1,55.8,56.5,56.6,101.2,104.4,112.4,113.3,114.6$, 116.0, 128.8, 129.6, 130.8, 150.1, 150.8, 159.5, 165.5; IR (neat, $\mathrm{cm}^{-1}$ ) 3387, 2958, 1653, 1511; HRMS Calcd for $\mathrm{C}_{22} \mathrm{H}_{27} \mathrm{NO}_{4} \mathrm{Si}$ : 397.1709. Found: 397.1713 .

\section{(3E)-3-[Iodo(trimethylsilyl)methylene]-5,6-dimethoxy-2-(4-}

methoxybenzyl)isoindolin-1-one (53). This compound was prepared by following the general procedure for the electrophilic cyclization of alkynylarenecarboxamides by $\mathrm{I}_{2}$. Purification by flash chromatography (1:1 hexane/EtOAc) afforded $126 \mathrm{mg}(80 \%)$ of the product as a white solid: $\operatorname{mp} 122-123{ }^{\circ} \mathrm{C} ;{ }^{1} \mathrm{H} \mathrm{NMR}\left(\mathrm{CDCl}_{3}\right) \delta 0.39(\mathrm{~s}, 9 \mathrm{H}), 3.79(\mathrm{~s}, 3 \mathrm{H})$, $3.95(\mathrm{~s}, 3 \mathrm{H}), 3.99(\mathrm{~s}, 3 \mathrm{H}), 4.72(\mathrm{~s}, 2 \mathrm{H}), 6.88(\mathrm{dd}, J=1.8,6.9 \mathrm{~Hz}, 2 \mathrm{H}), 7.30-7.34(\mathrm{~m}, 3 \mathrm{H})$, $8.42(\mathrm{~s}, 1 \mathrm{H}) ;{ }^{13} \mathrm{C} \mathrm{NMR}\left(\mathrm{CDCl}_{3}\right) \delta 0.0,50.9,54.7,55.7,55.8,103.5,106.8,113.3,125.2$, $128.4,129.6,131.6,151.2,153.6,154.6,158.0$ (two carbons missing due to overlap); IR 
(neat, $\mathrm{cm}^{-1}$ ) 3002, 2955, 1698, 1512; HRMS Calcd for $\mathrm{C}_{22} \mathrm{H}_{26} \mathrm{INO}_{4} \mathrm{Si}$ : 523.0676. Found: 523.0686 .

(3E)-3-(2-Bromobenzylidene)-5,6-dimethoxy-2-(4-methoxybenzyl)isoindolin-1-one (54). Compound 53 (366 mg, $0.7 \mathrm{mmol}), \mathrm{KF}$ (43 mg, 1.05 equiv) and $\mathrm{H}_{2} \mathrm{O}$ (14 mg) were dissolved in DMF (4.5 ml) and the resulting reaction mixture was stirred at room temperature for $0.5 \mathrm{~h}$. The mixture was then diluted with $\mathrm{Et}_{2} \mathrm{O}(100 \mathrm{ml})$, washed with $\mathrm{H}_{2} \mathrm{O}(3 \times 25 \mathrm{ml})$, dried $\left(\mathrm{MgSO}_{4}\right)$ and filtered. The solvent was evaporated under reduced pressure and this crude intermediate was used in the next step without any further purification.

The intermediate, 2-bromophenylboronic acid (168.5 mg, 1.2 equiv), $\mathrm{PPh}_{3}$ (18.4 mg), CsF (257 mg, 2.4 equiv) and DMF (3.7 ml) were added to a 2-dram vial and the resulting mixture was then stirred under an $\mathrm{N}_{2}$ atm at room temperature for $30 \mathrm{~h}$. The reaction mixture was diluted with $\mathrm{CHCl}_{3}(100 \mathrm{ml})$, washed with satd aq $\mathrm{NH}_{4} \mathrm{Cl}(25 \mathrm{ml})$, dried $\left(\mathrm{MgSO}_{4}\right)$ and filtered. The solvent was evaporated under reduced pressure and the product was isolated by flash chromatography (1:1 hexane/EtOAc) affording $190 \mathrm{mg}$ (57 $\%)$ of the product as a yellow solid: $\mathrm{mp} 122-124{ }^{\circ} \mathrm{C} ;{ }^{1} \mathrm{H} \mathrm{NMR}\left(\mathrm{CDCl}_{3}\right) \delta 3.60(\mathrm{~s}, 3 \mathrm{H})$, $3.80(\mathrm{~s}, 3 \mathrm{H}), 3.91(\mathrm{~s}, 3 \mathrm{H}), 4.81(\mathrm{~s}, 2 \mathrm{H}), 6.52(\mathrm{~s}, 1 \mathrm{H}), 6.61(\mathrm{~s}, 1 \mathrm{H}), 6.91(\mathrm{~d}, J=8.4 \mathrm{~Hz}$, 2H), 7.22-7.28 (m, 2H), 7.34-7.41 (m, 3H), $7.61(\mathrm{~d}, J=6.9 \mathrm{~Hz}, 1 \mathrm{H}), 7.71(\mathrm{dd}, J=0.9,8.1$ $\mathrm{Hz}, 1 \mathrm{H}) ;{ }^{13} \mathrm{C} \mathrm{NMR}\left(\mathrm{CDCl}_{3}\right) \delta 51.4,55.5,56.0,56.6,104.2,104.3,105.1,114.1,125.3$, $125.5,127.2,128.7,129.4,129.6,131.7,132.7,133.4,135.1,150.6,151.8,152.3,158.8$ (one carbon missing due to overlap); IR (neat, $\mathrm{cm}^{-1}$ ) 3004, 2932, 1698, 1497; HRMS Calcd for $\mathrm{C}_{25} \mathrm{H}_{22} \mathrm{BrNO}_{4}$ : 479.0732. Found: 479.0741 . 


\section{References}

1. Kundu, N. G.; Khan, M. W. Tetrahedron 2000, 56, 4777.

2. Epsztajn, J.; Plotka, M. W.; Grabowska, A. Synth. Commun. 1997, 27, 1075.

3. Yao, T.; Larock, R. C. J. Org. Chem. 2003, 68, 5936. 

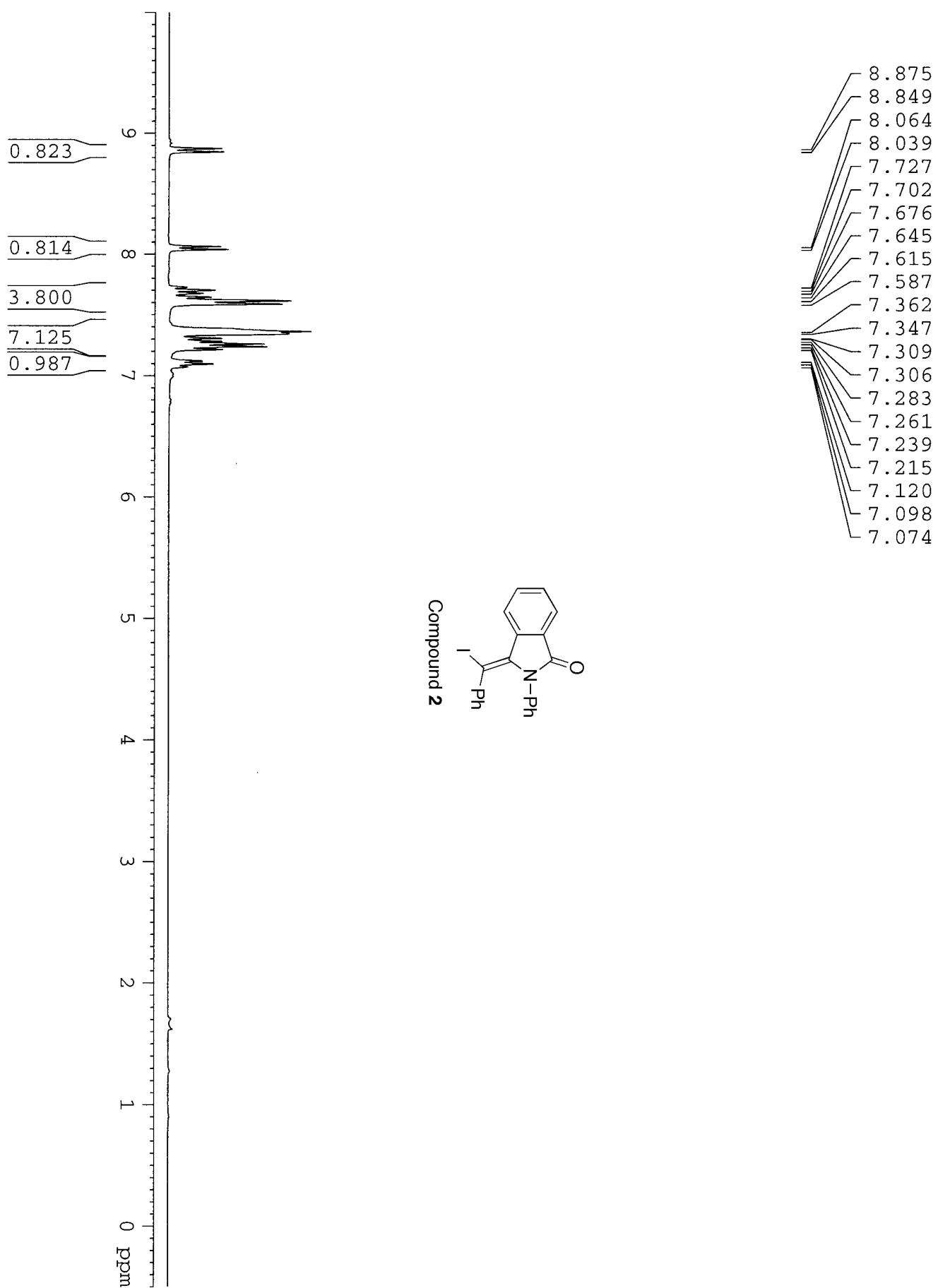

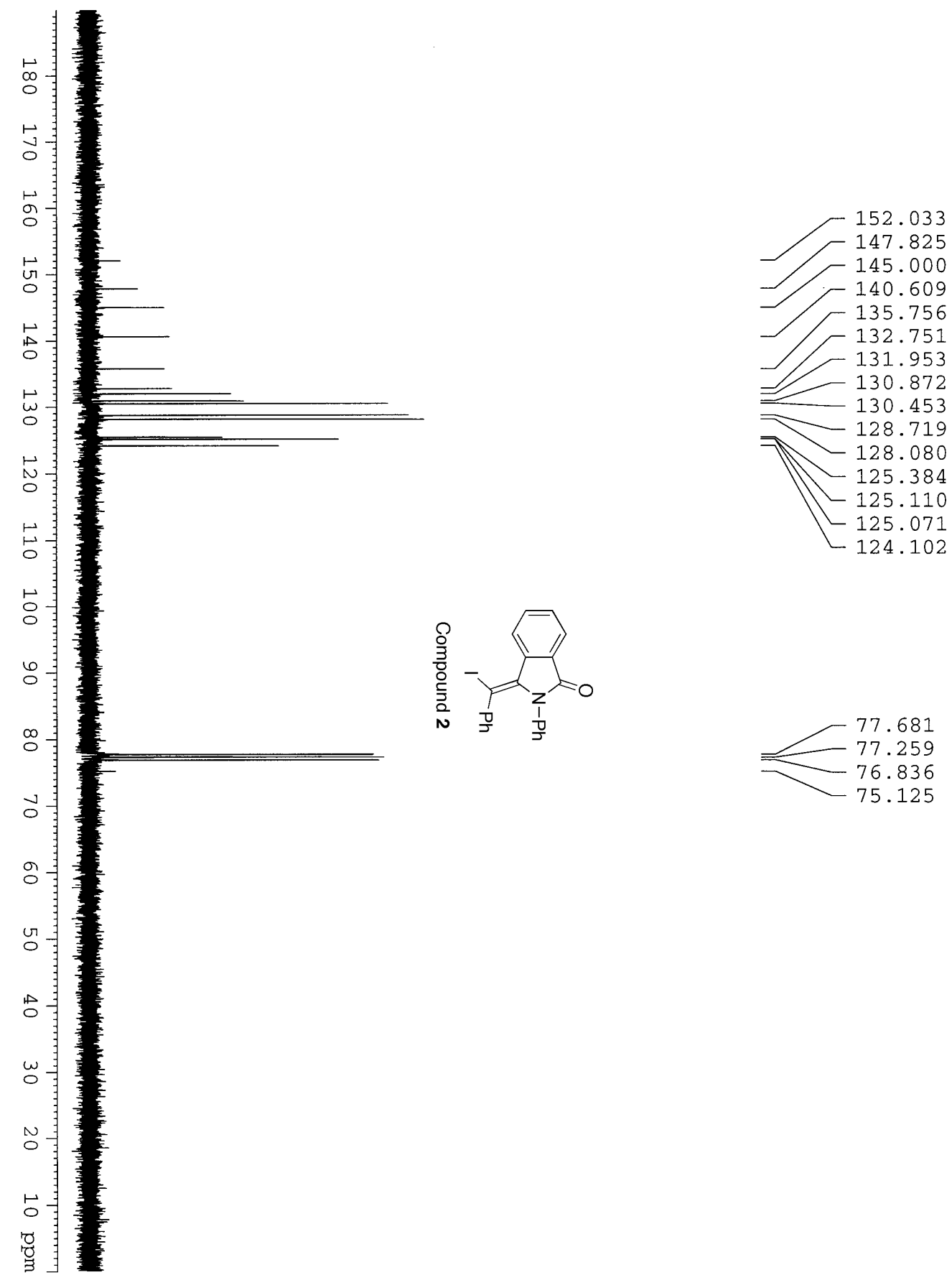

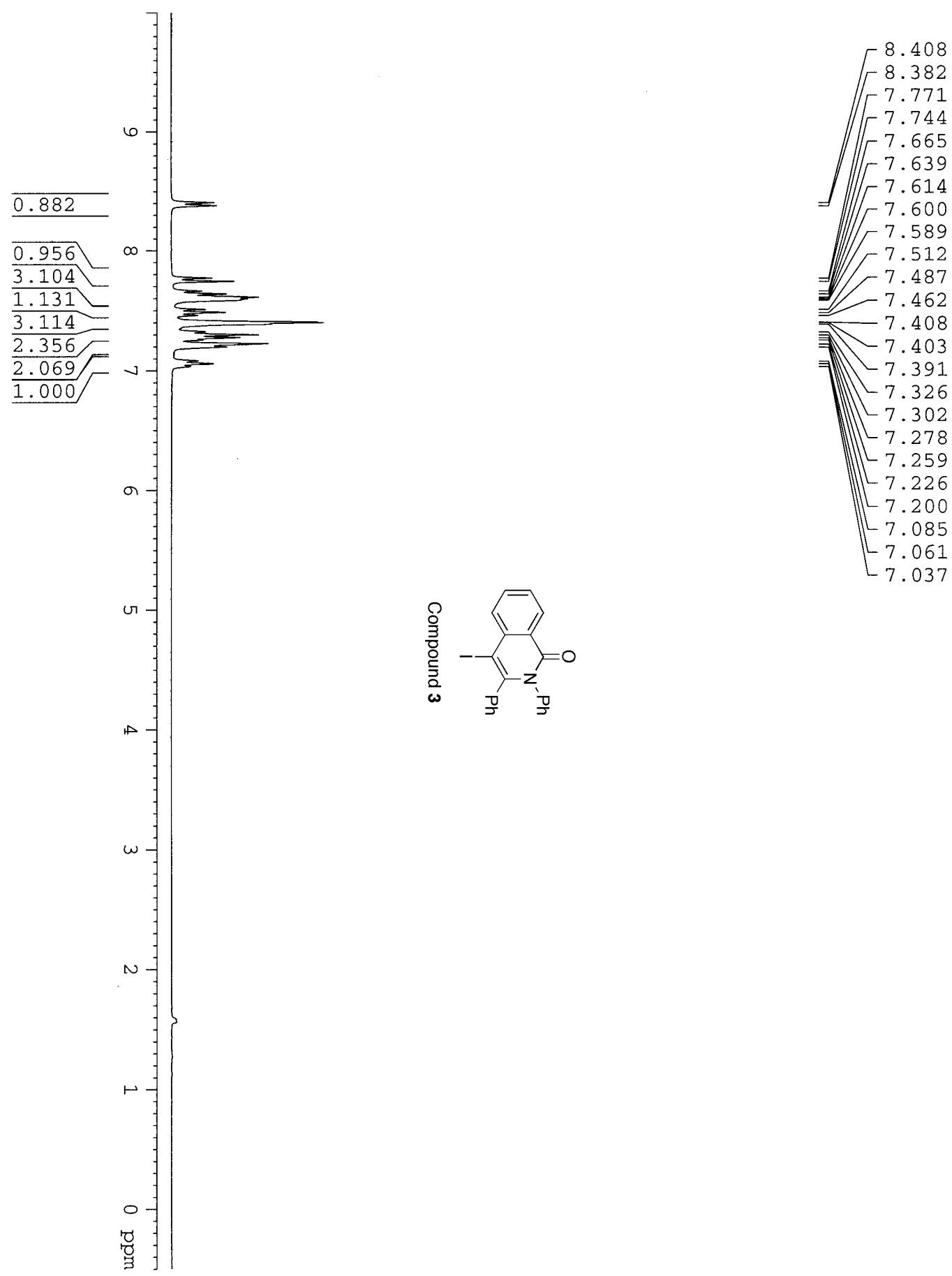

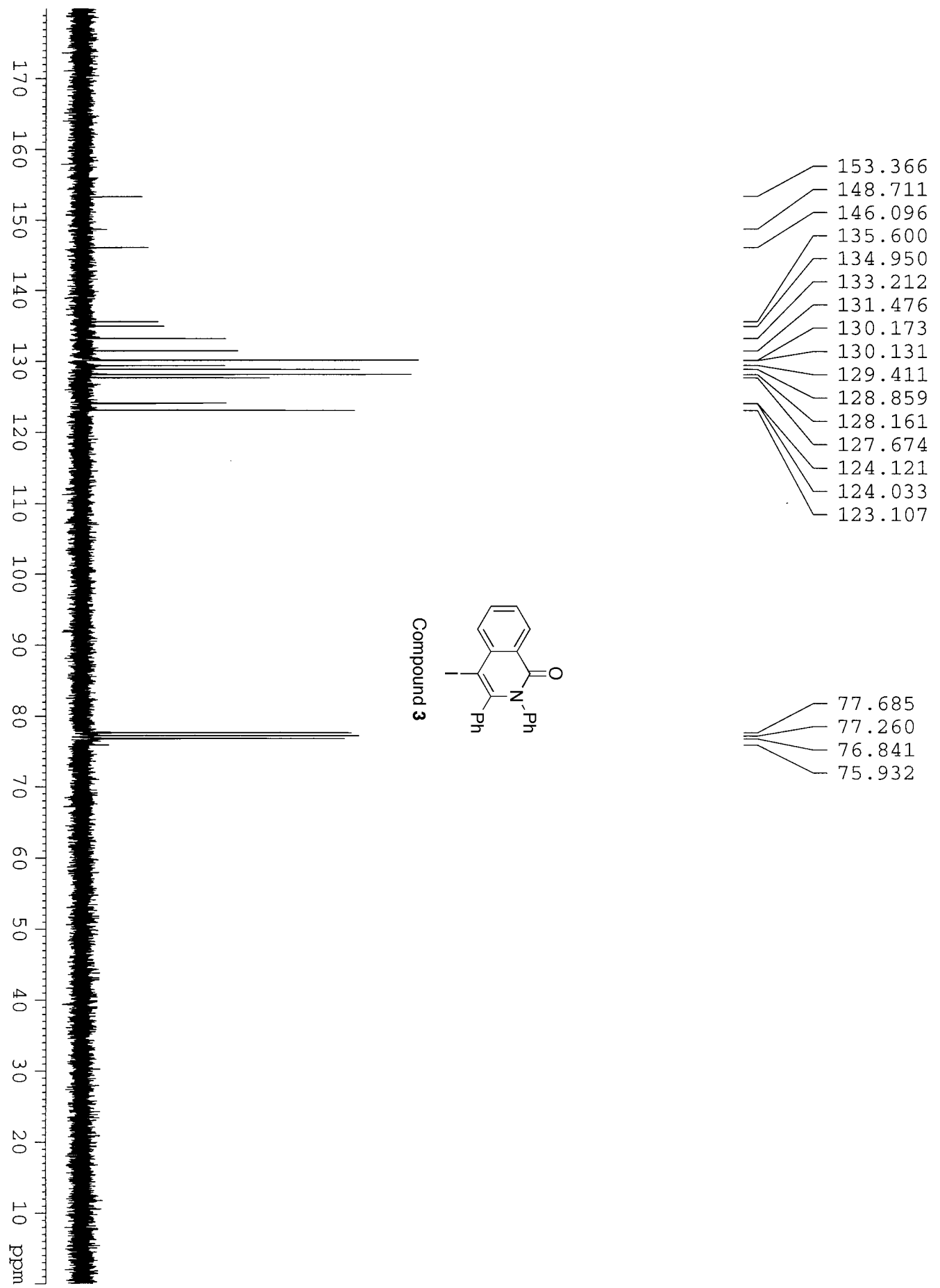

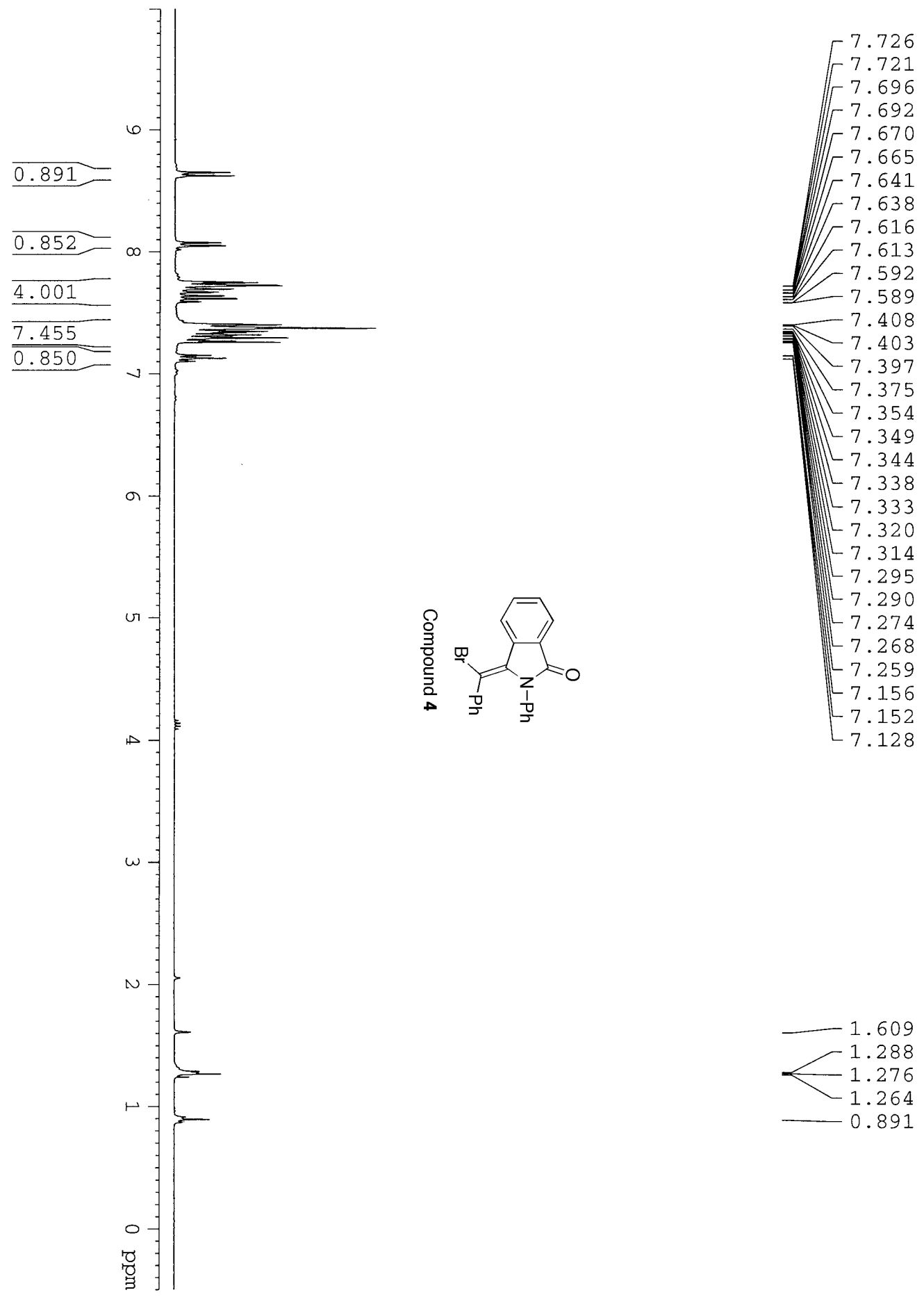

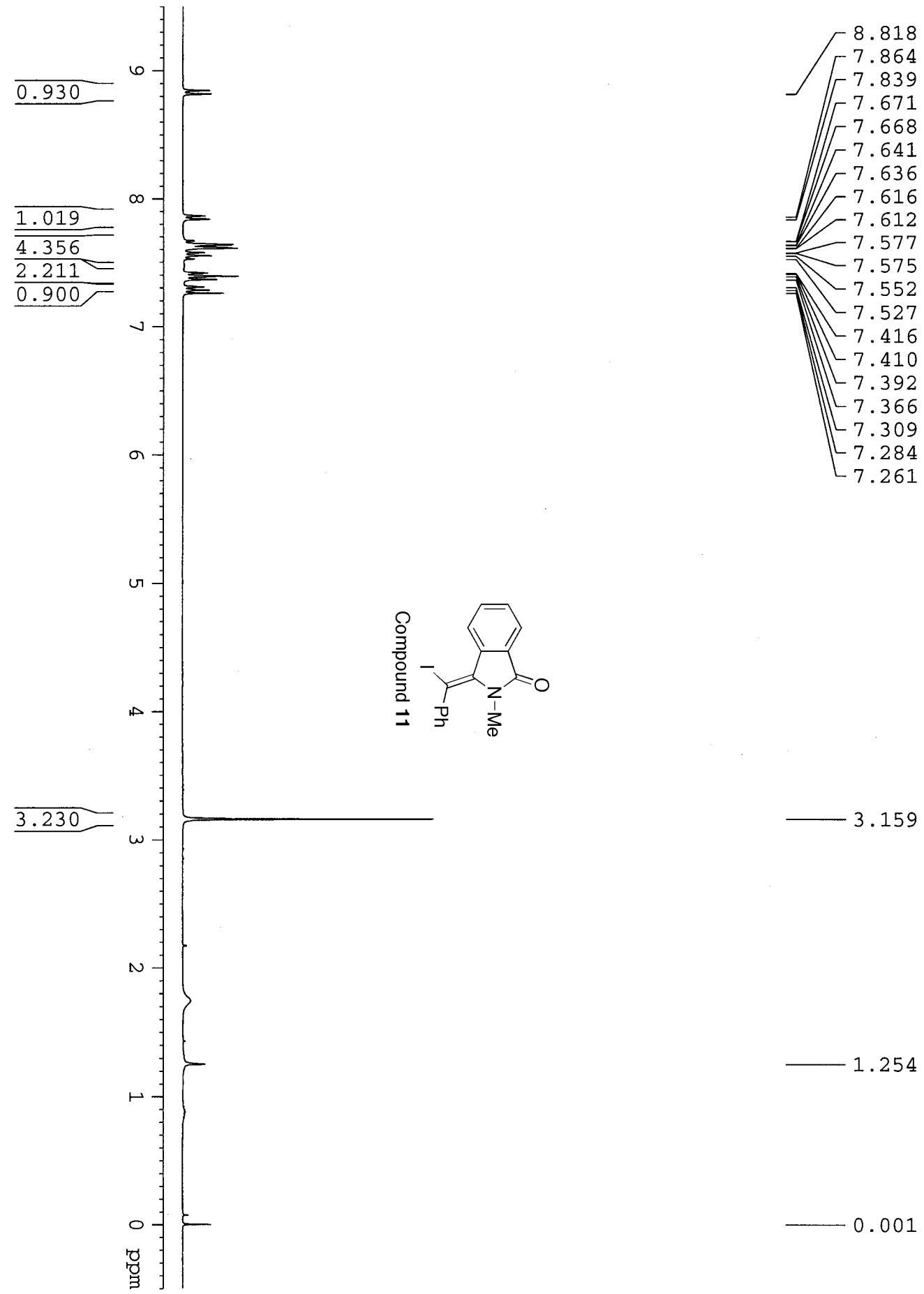

$-0.001$ 

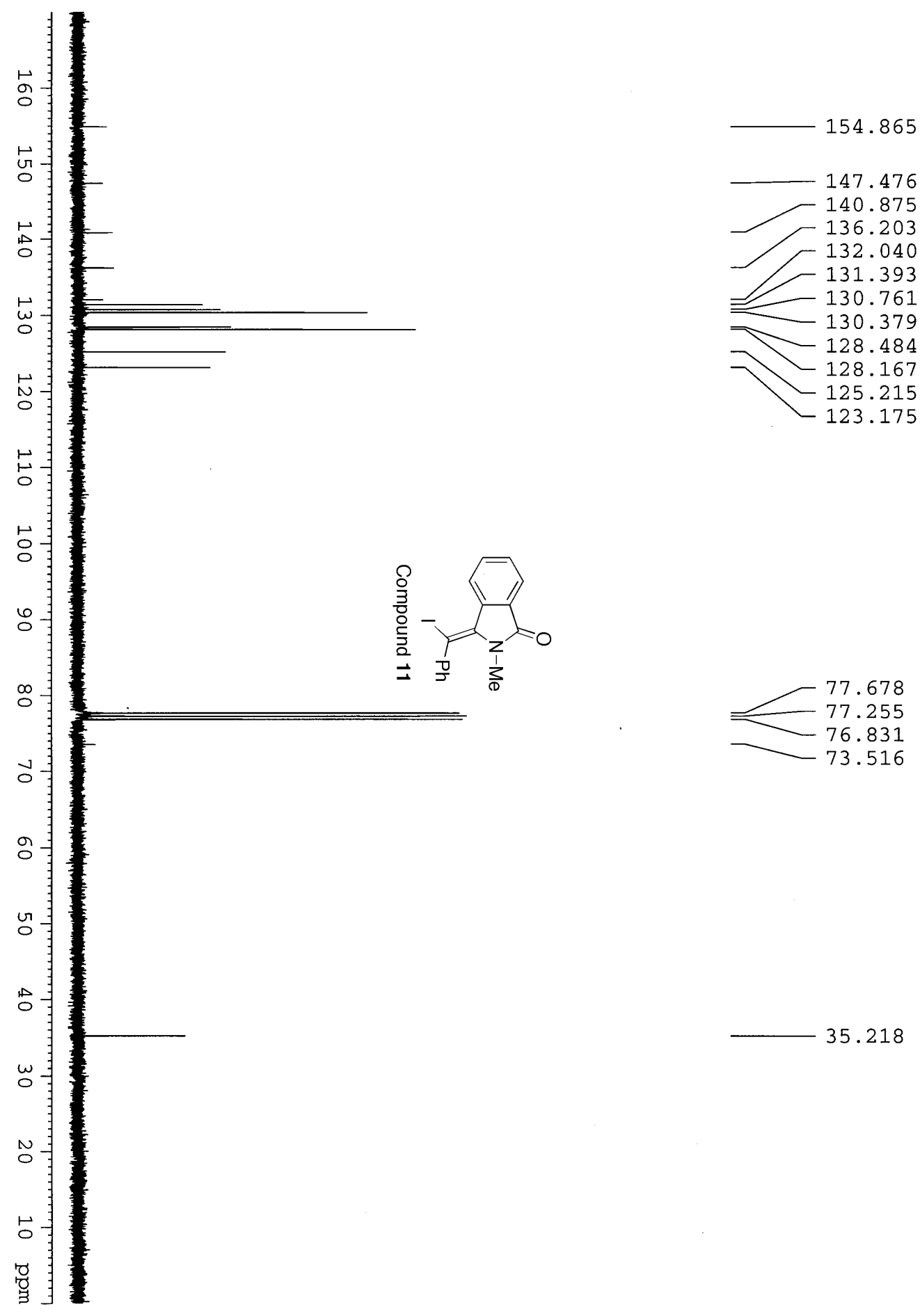

140.875

$-136.203$

$-132.040$

$-131.393$

$\simeq 130.761$

$-130.379$

$\longrightarrow 128.484$

$-128.167$

125.215

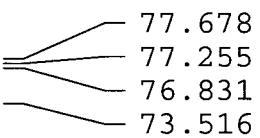

35.218 

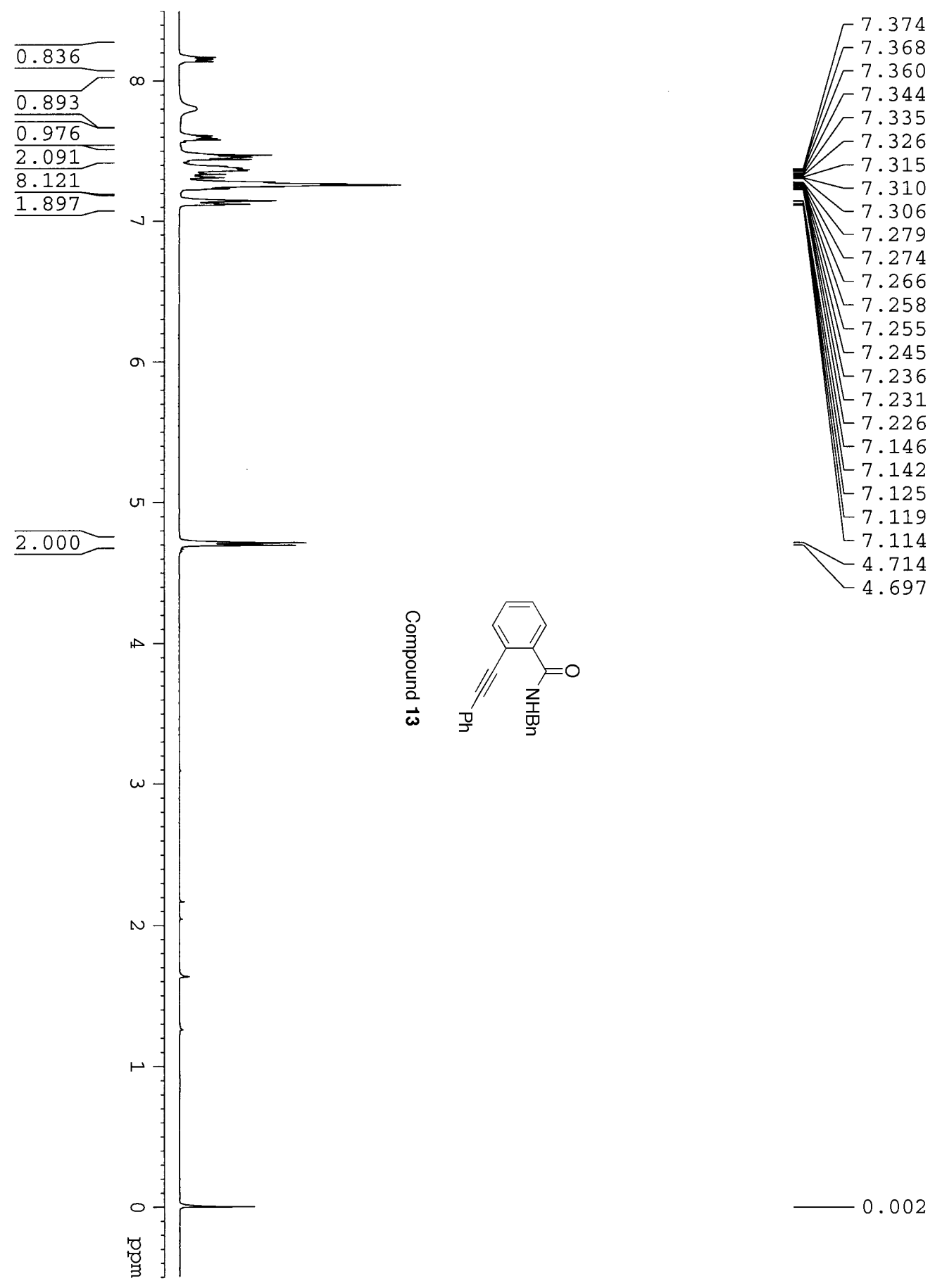


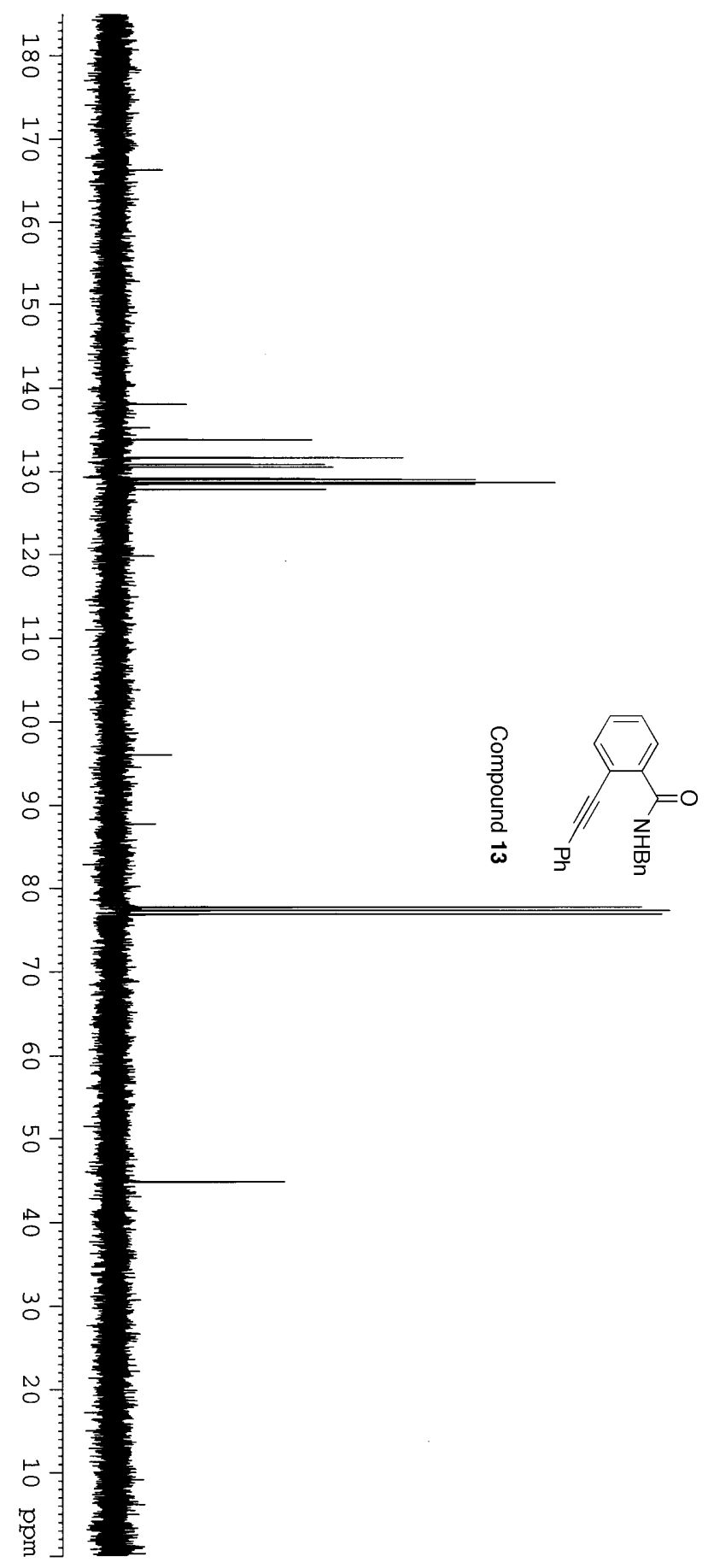

166.304
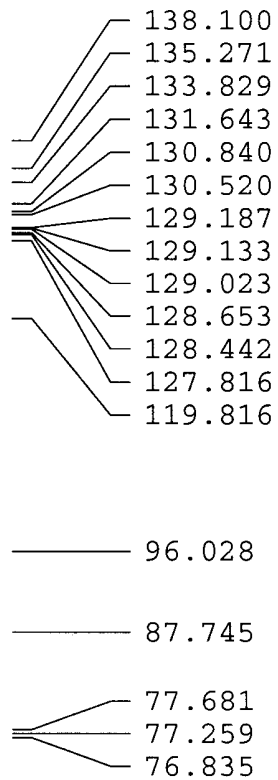

44.779 

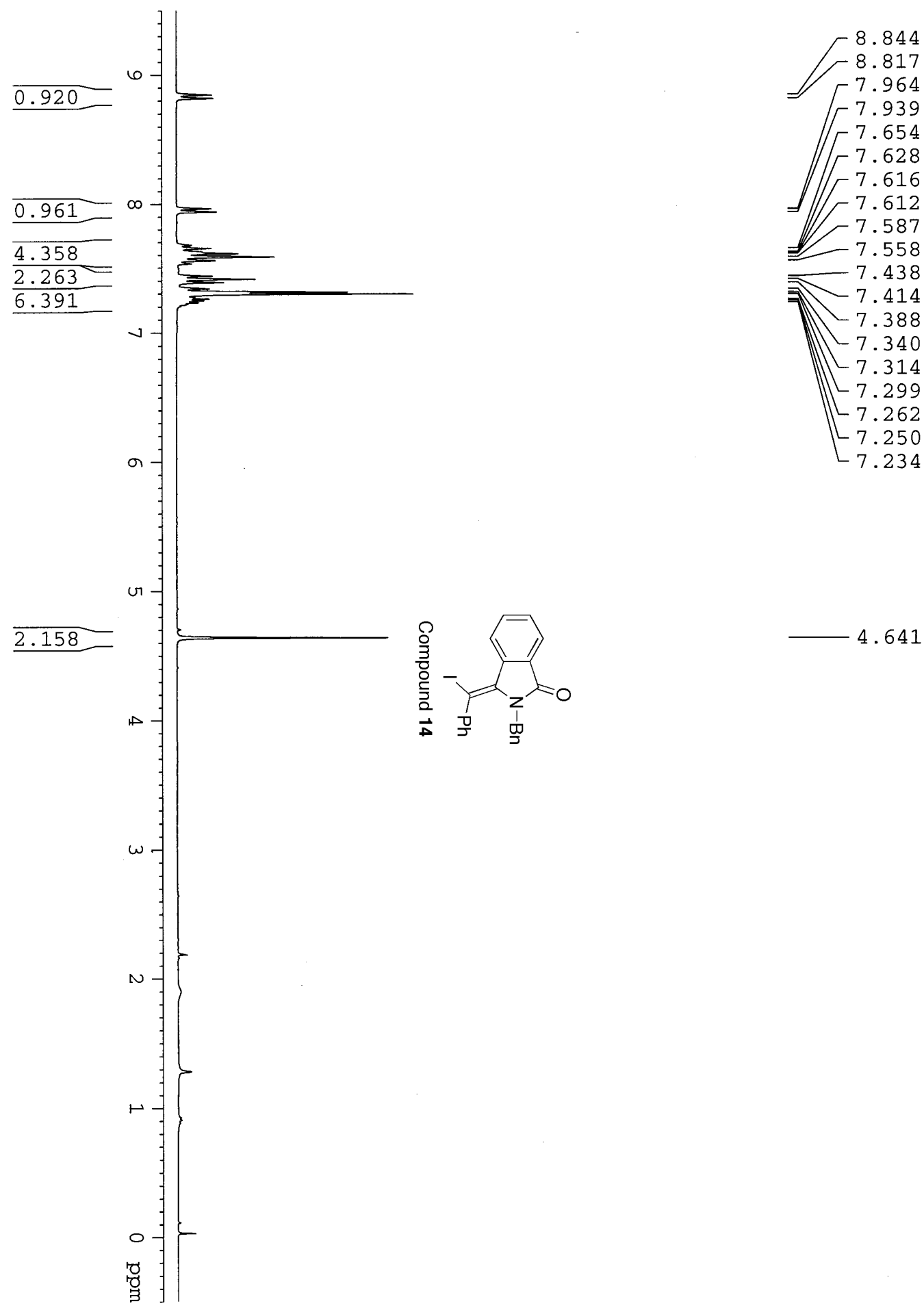

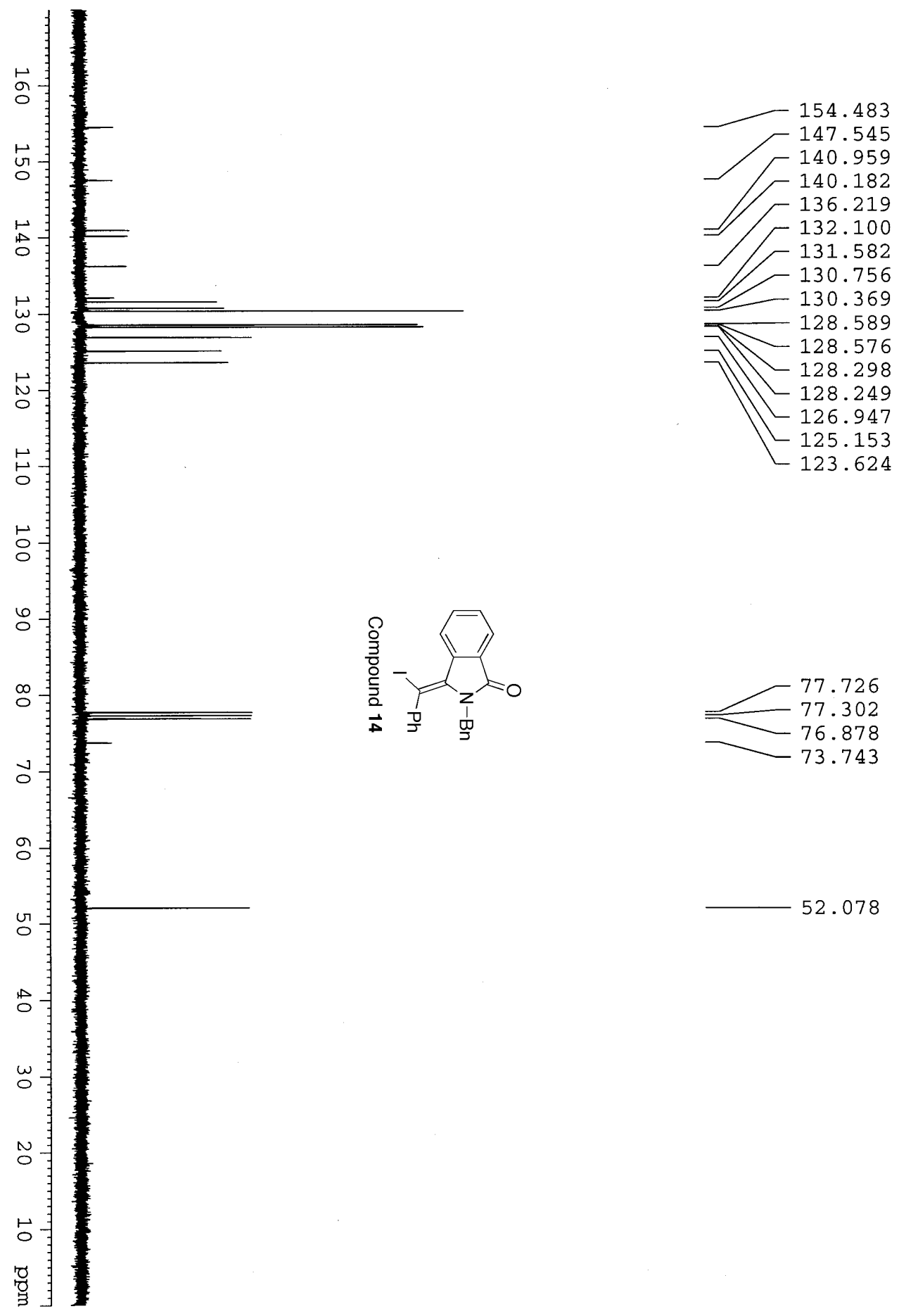

52.078 

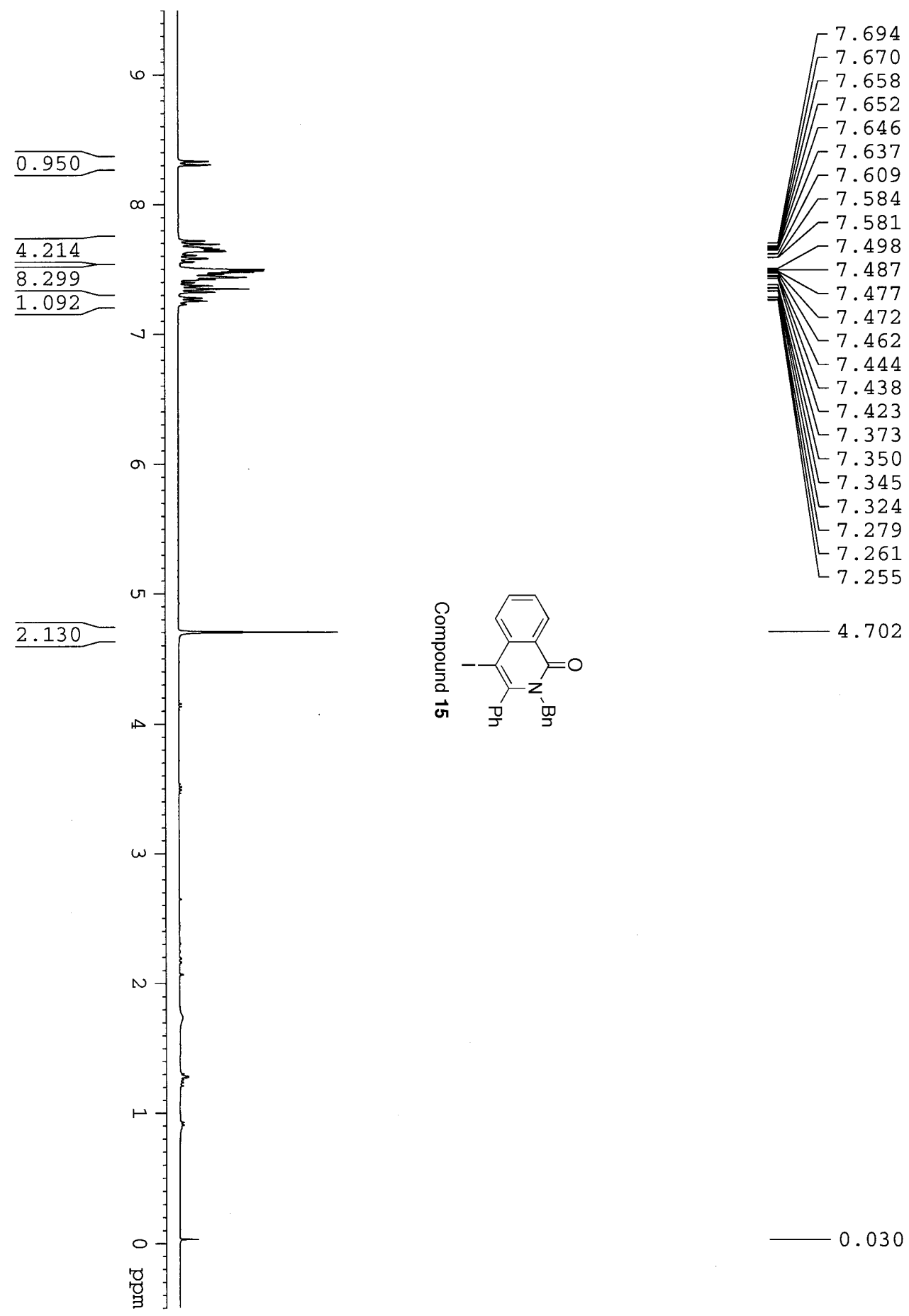

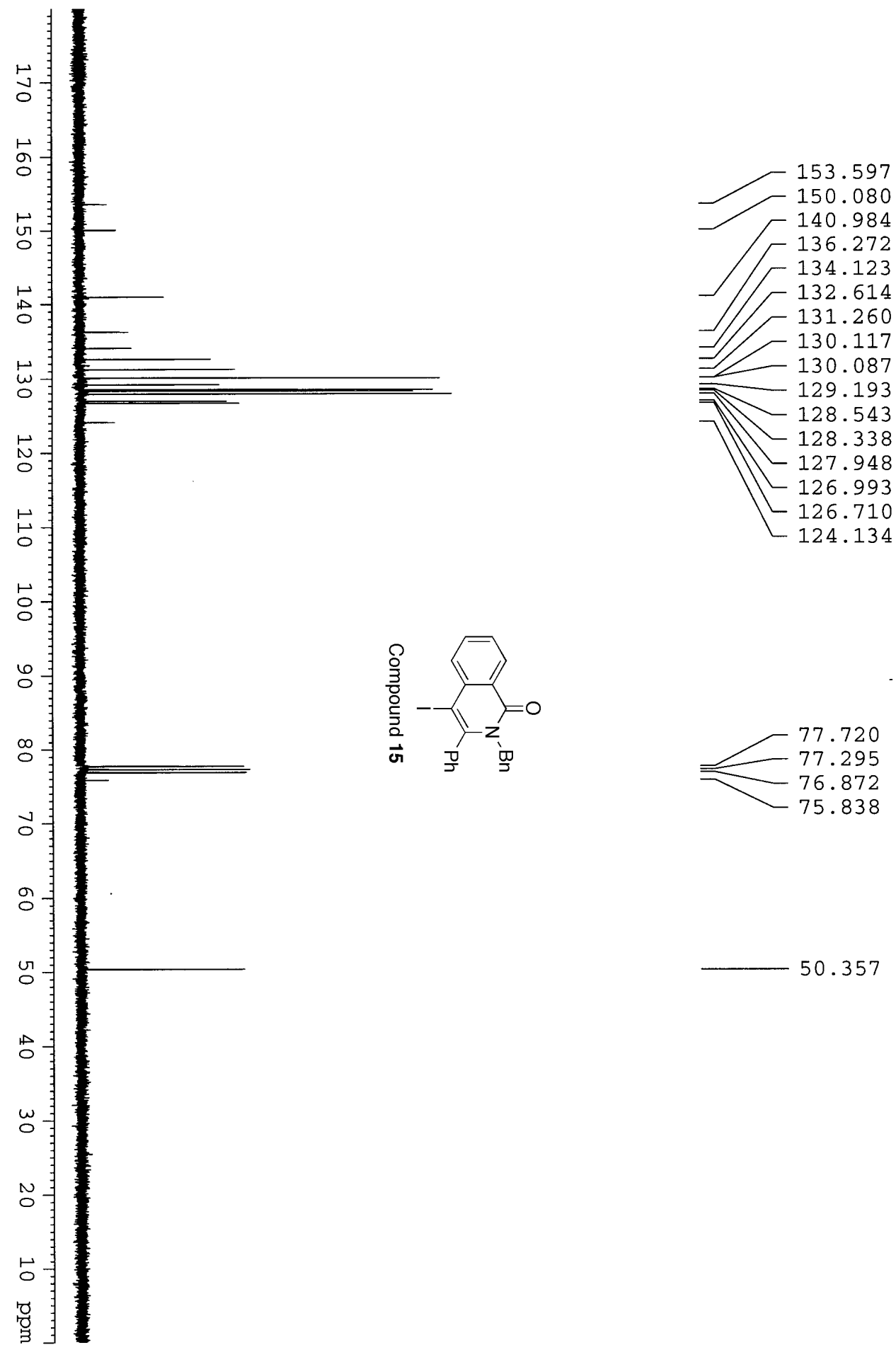

50.357 

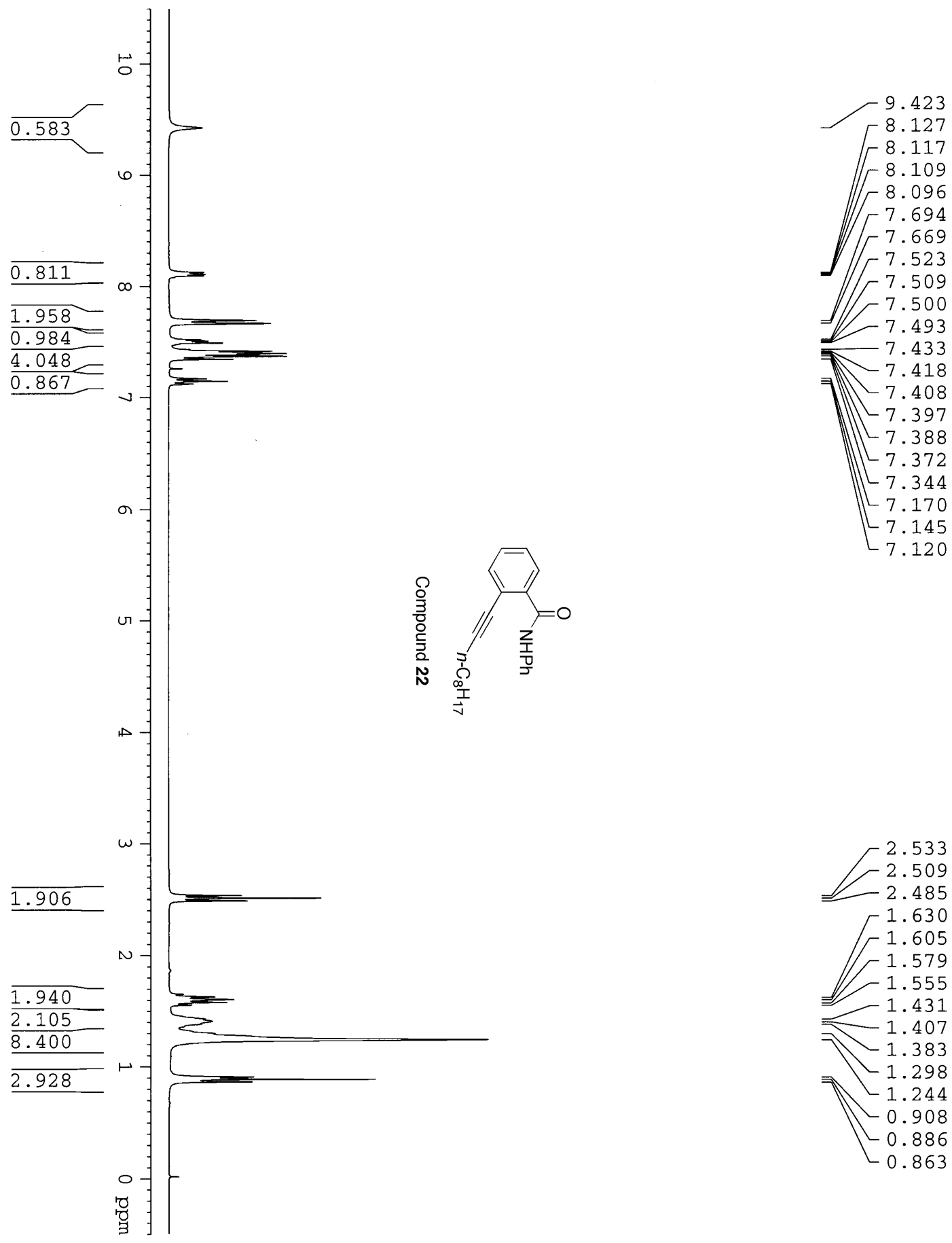

1.630

F 1.605

1.579

$-1.555$

$-1.431$

$\approx 1.407$

$-1.383$

$\approx 1.298$

1.244

$-0.908$

-0.886
-0.863 

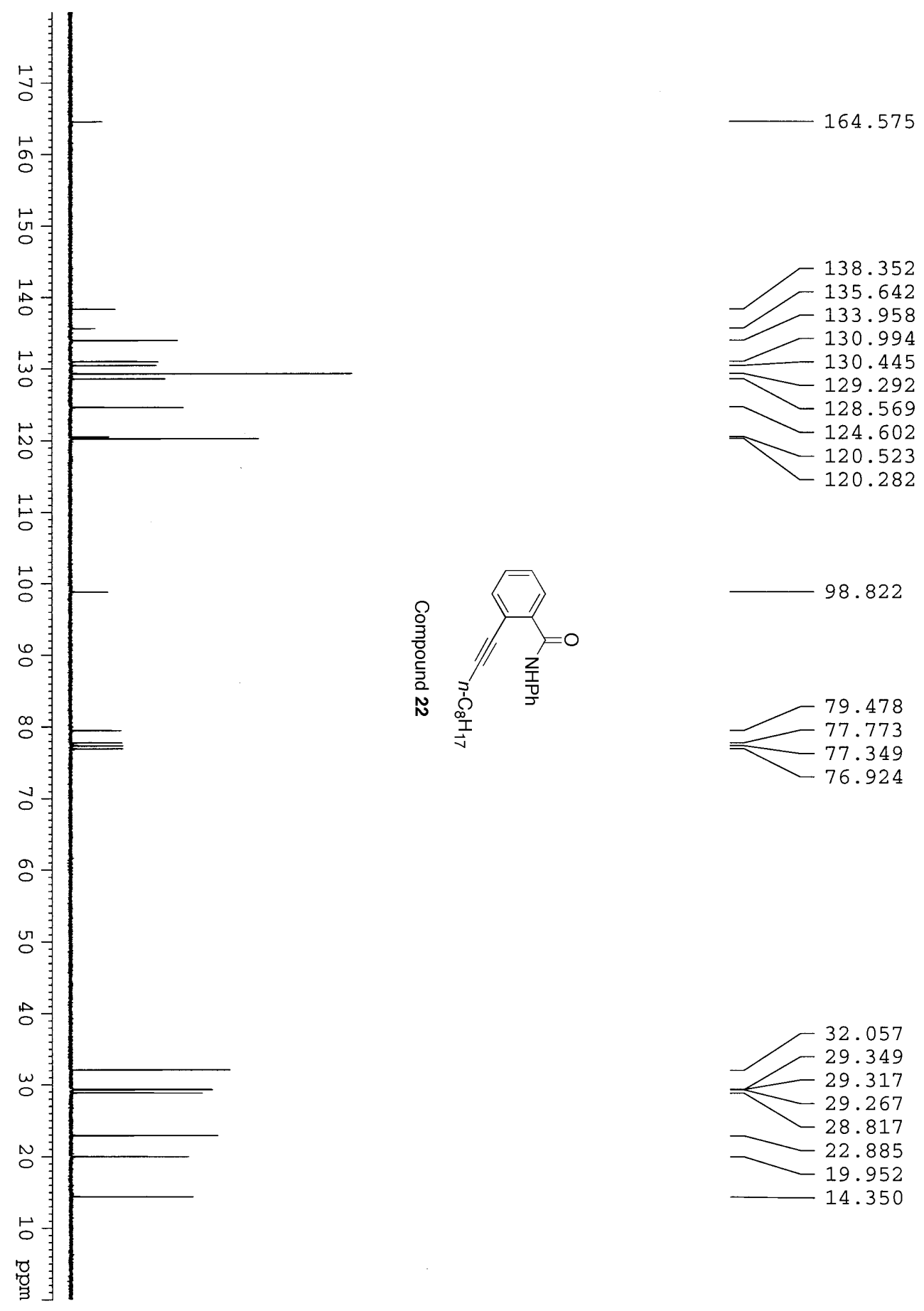

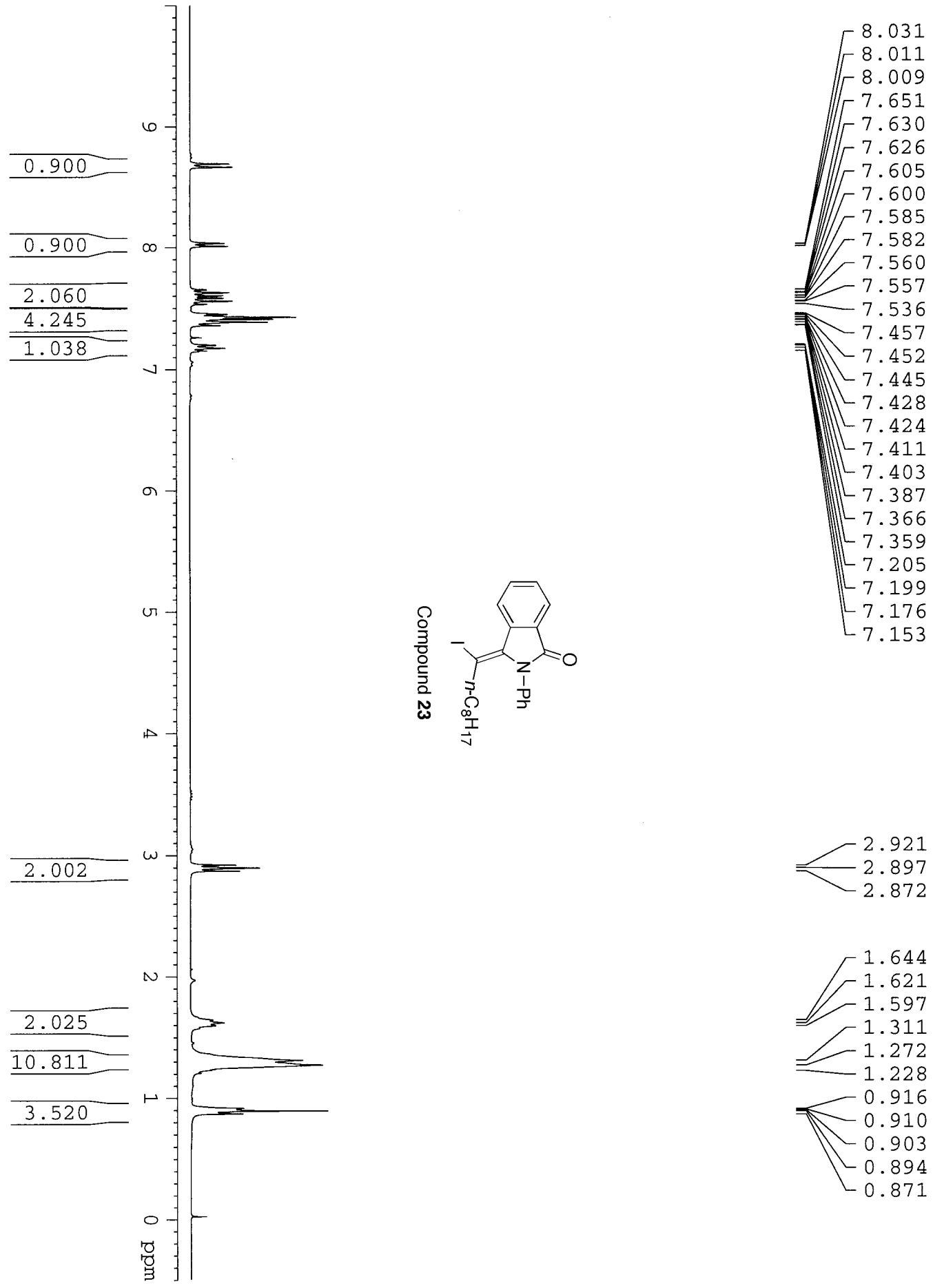

2.921

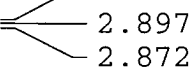

厂1.644

$\begin{array}{r}1.621 \\ -1.597 \\ \hline\end{array}$

$-1.311$

$-1.272$

$-1.228$

$-0.916$

0.910

$-0.903$

$-0.894$ 

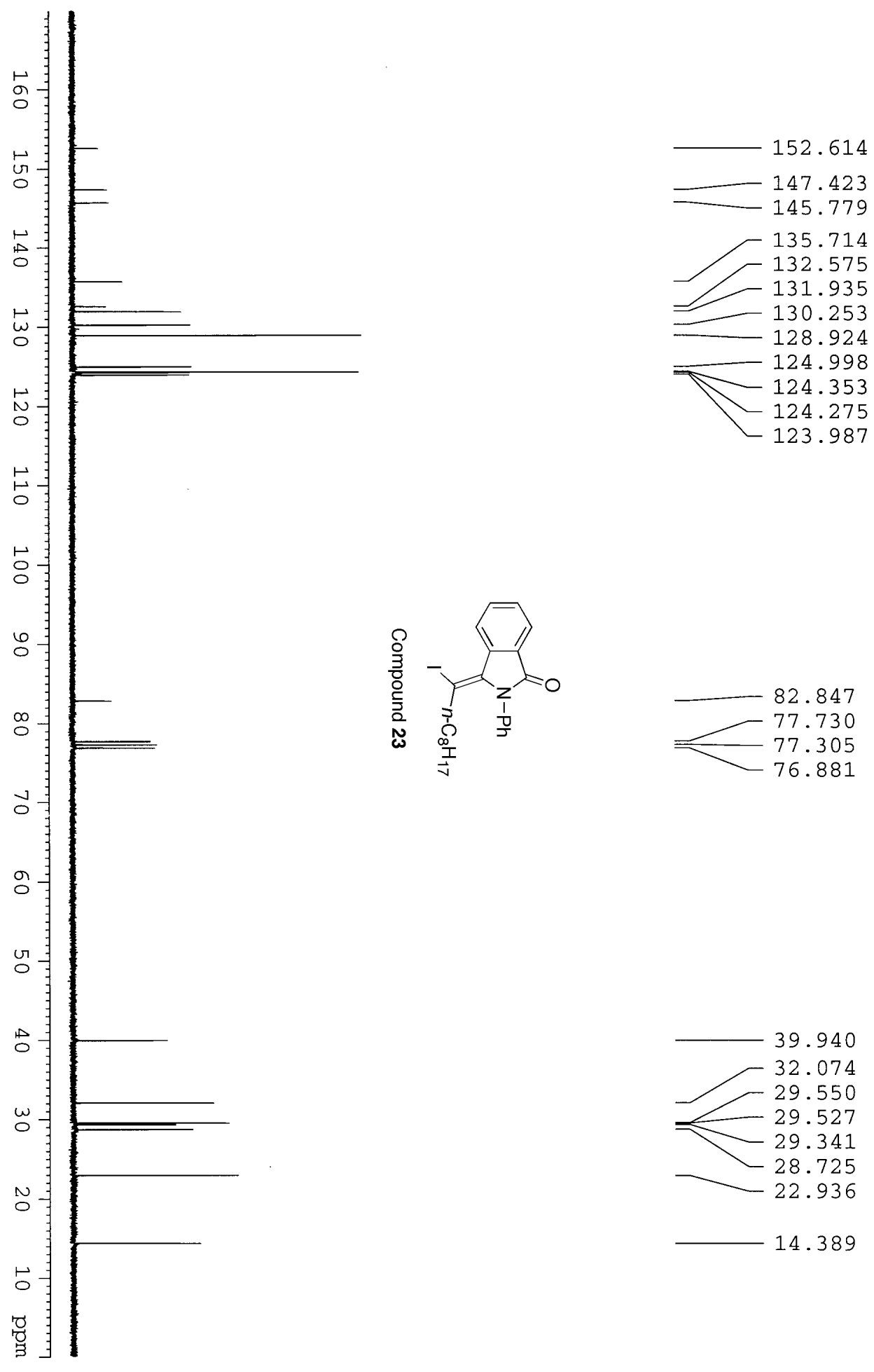


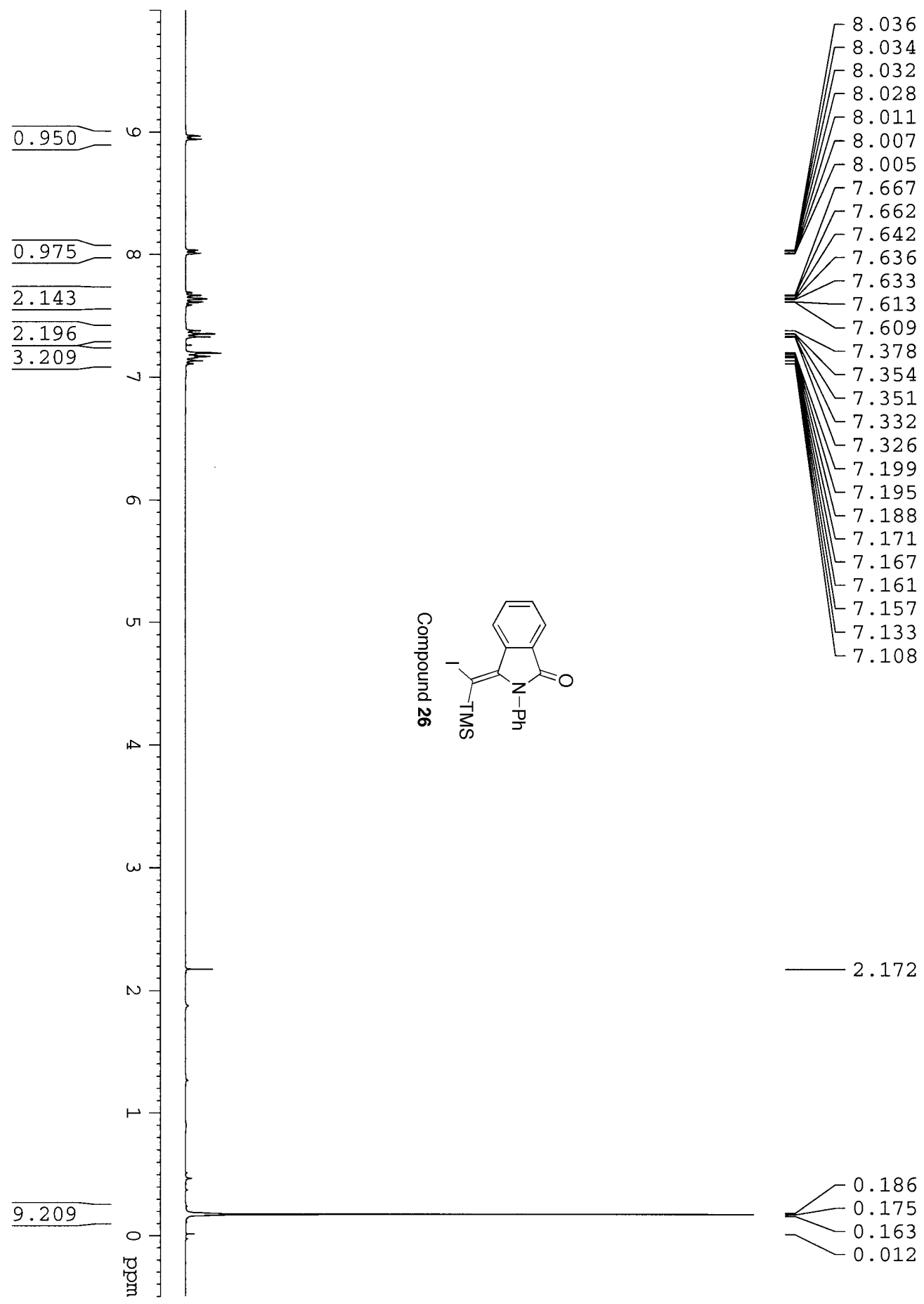




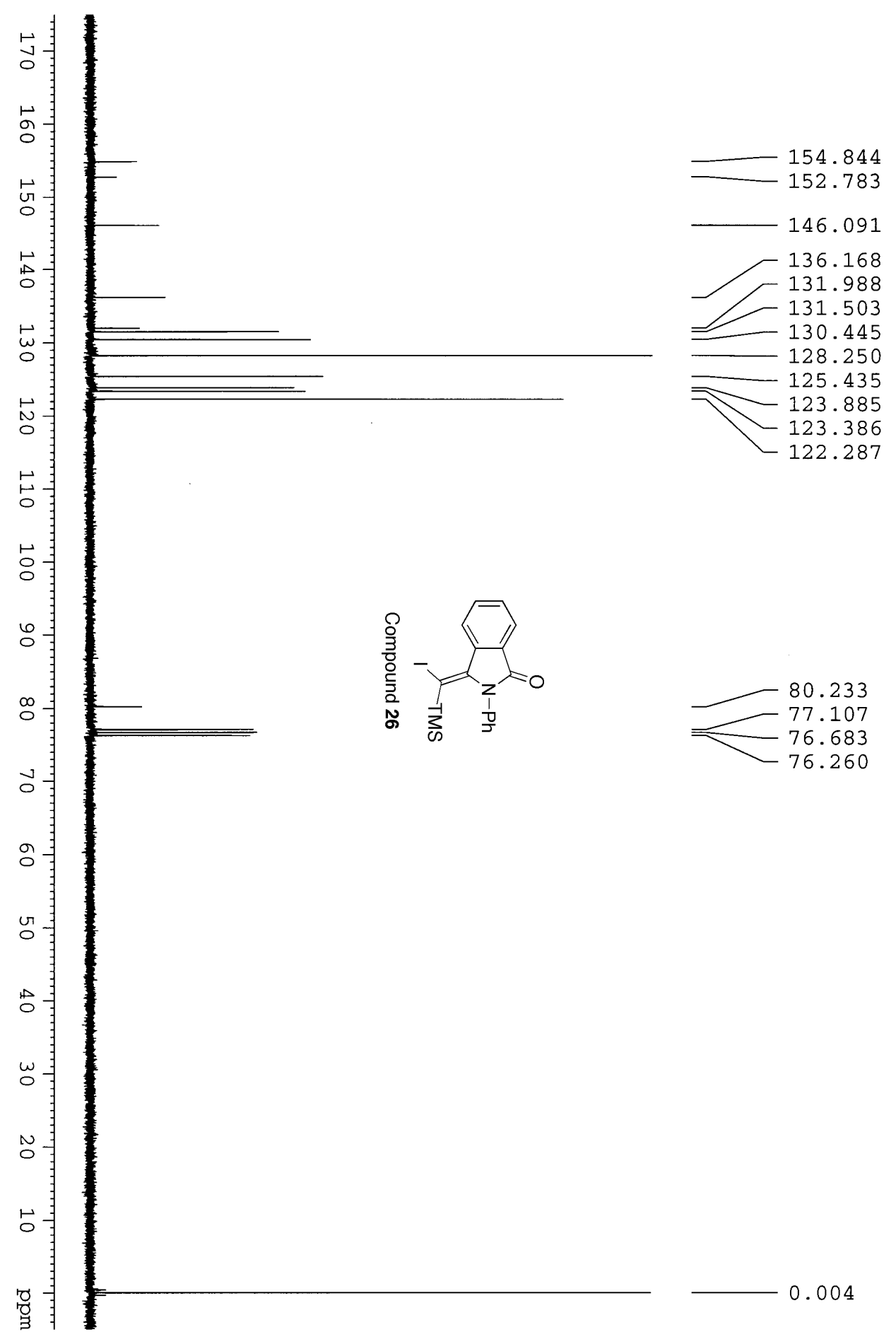



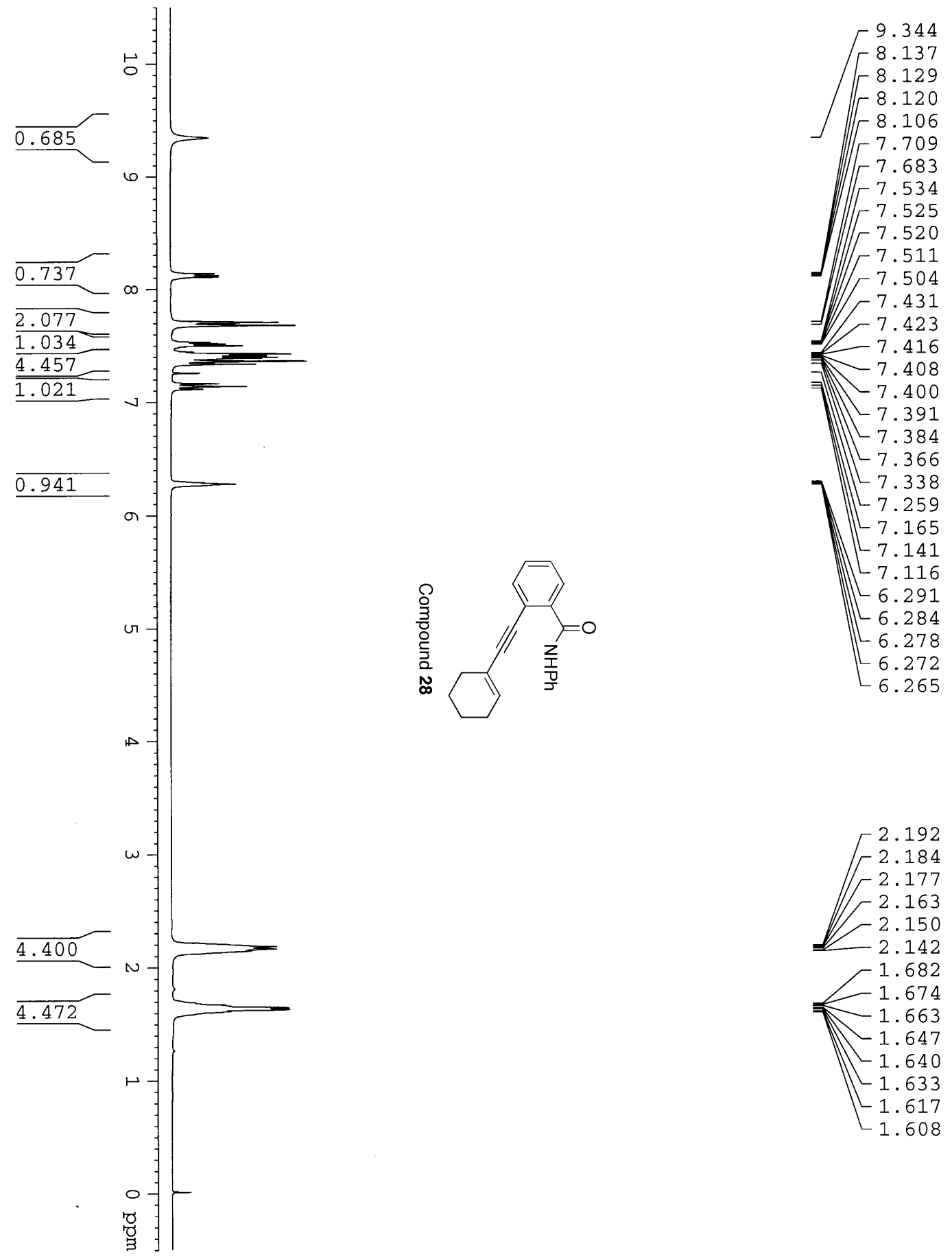

2.142

$-1.682$

$-1.674$

1.663

$-1.647$

$-1.640$

$-1.633$

$-1.617$

$-1.608$ 

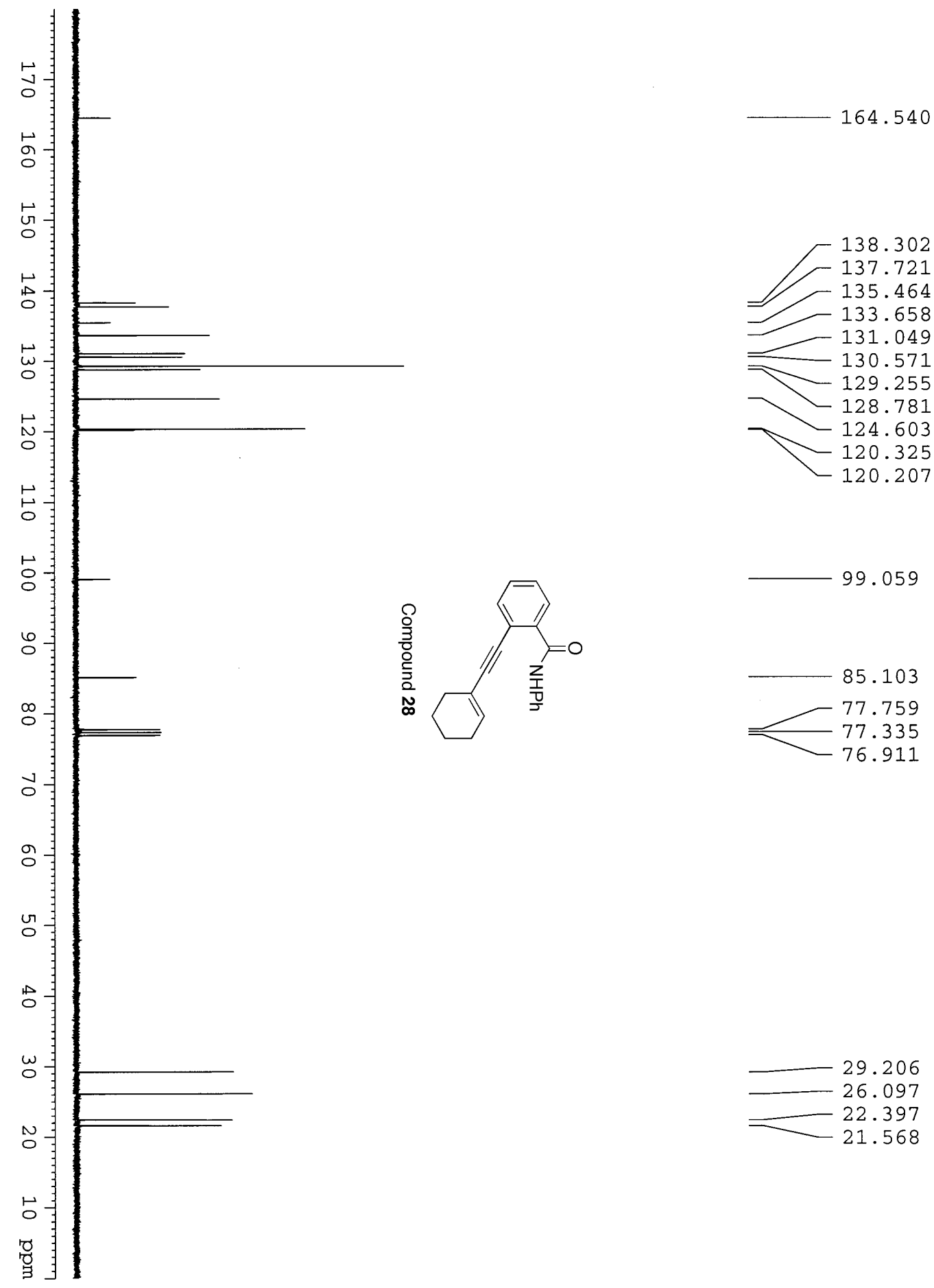

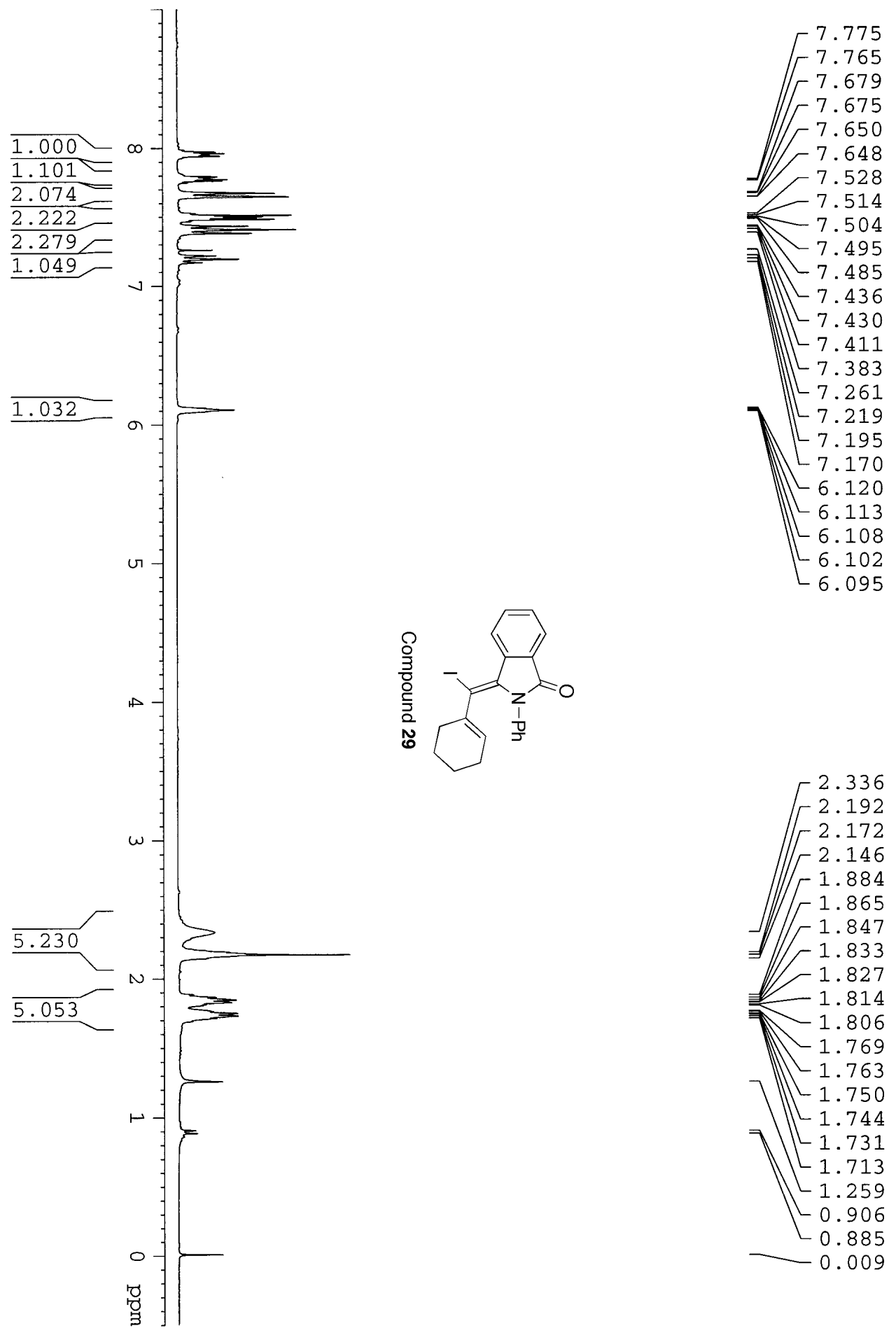

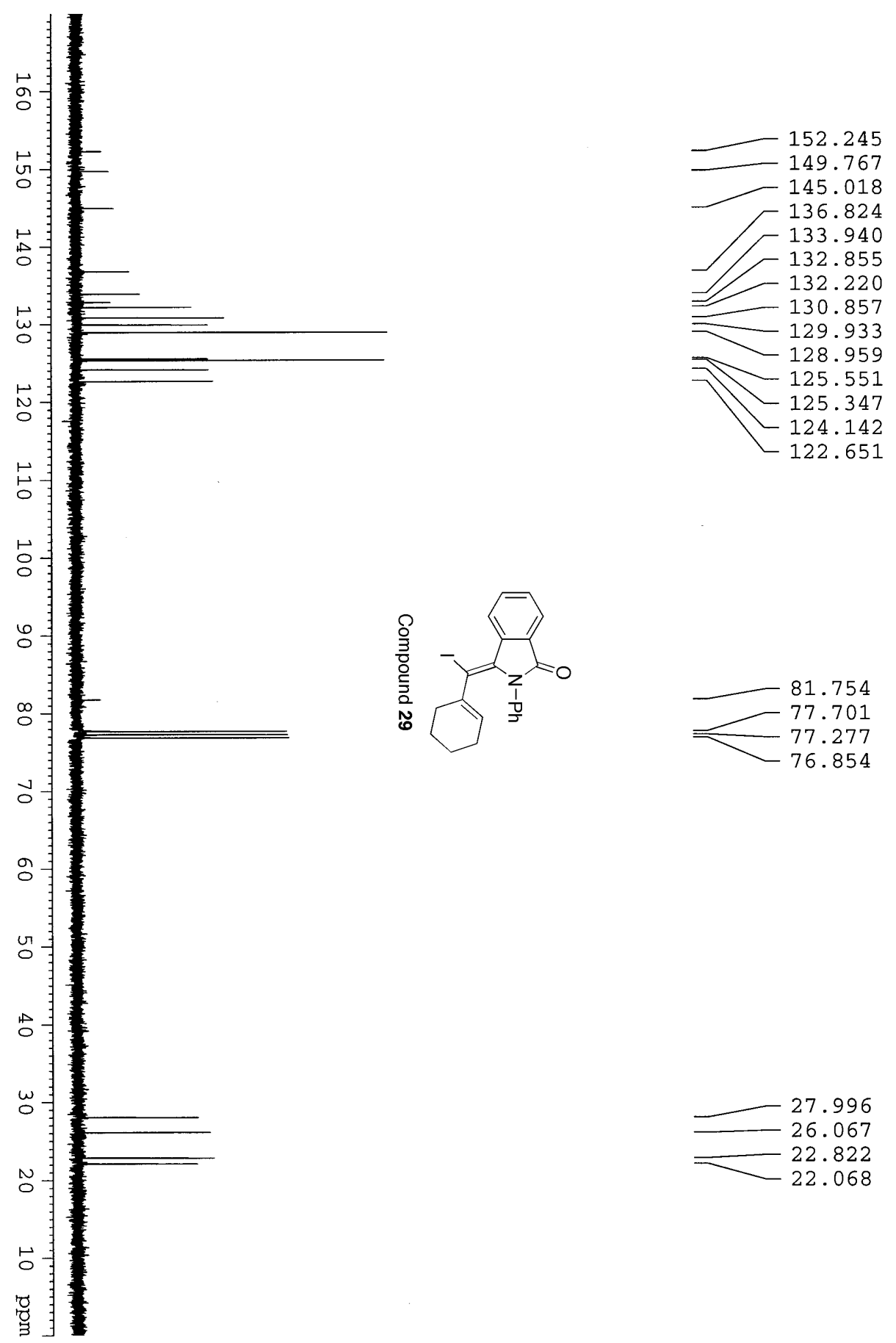

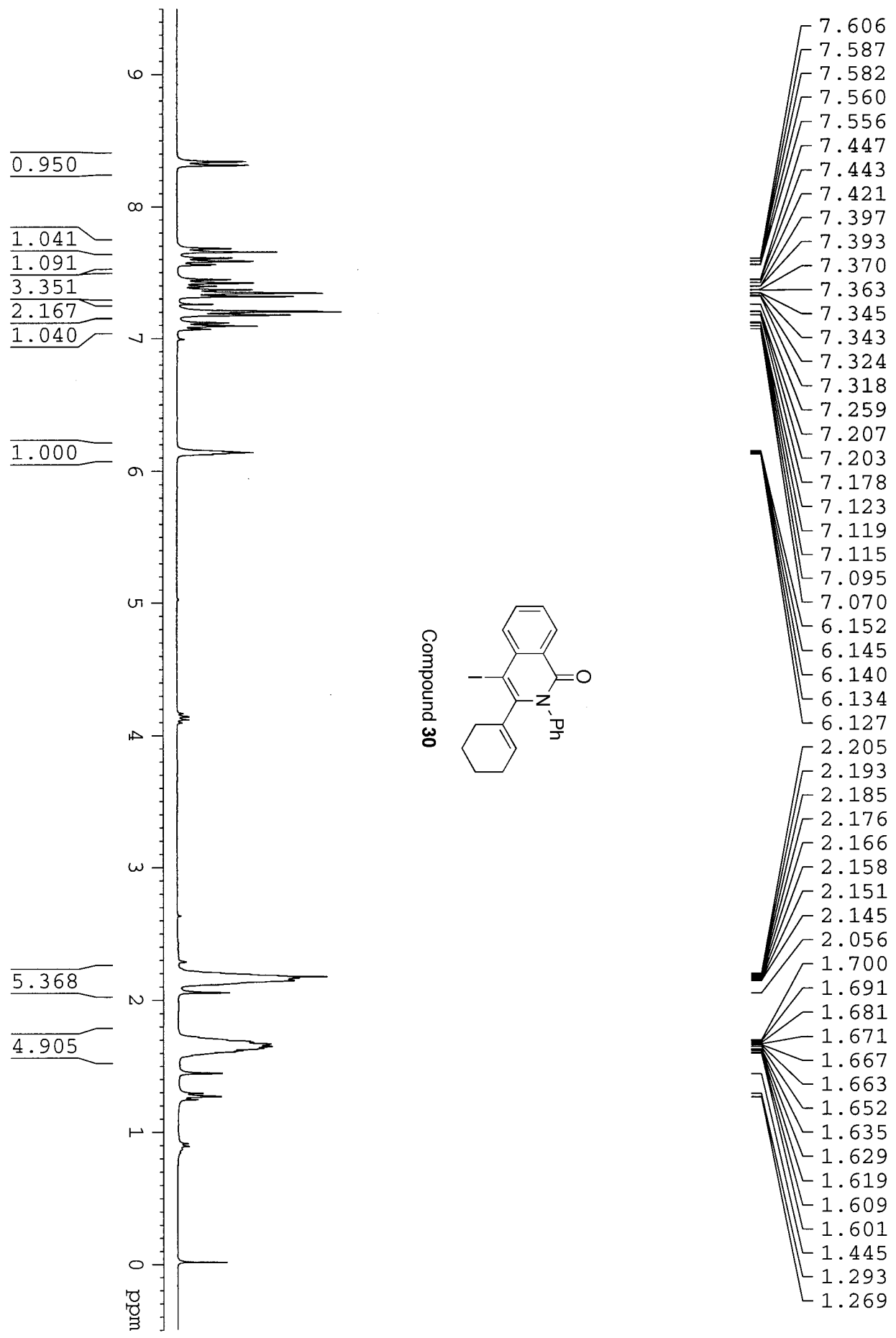

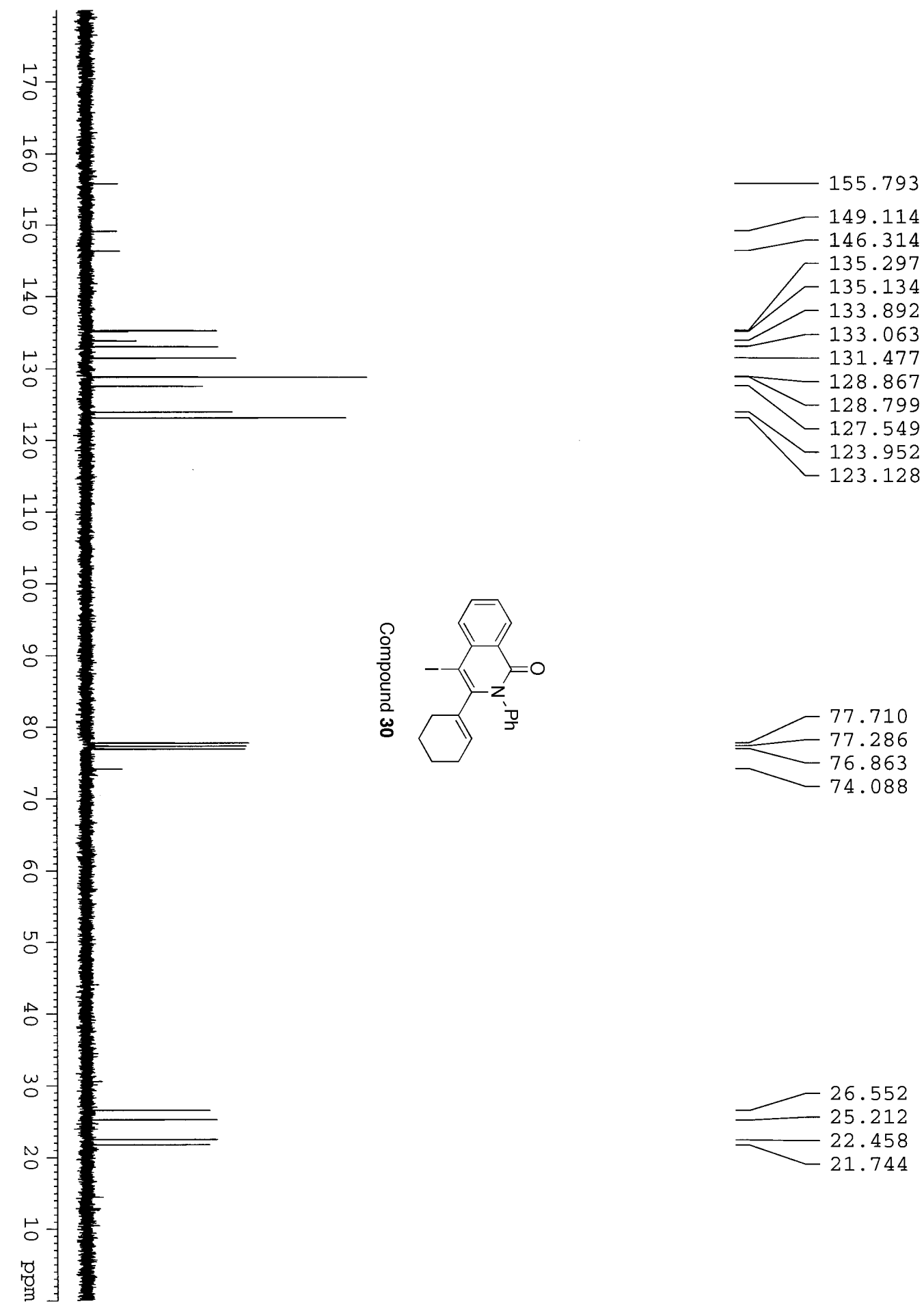

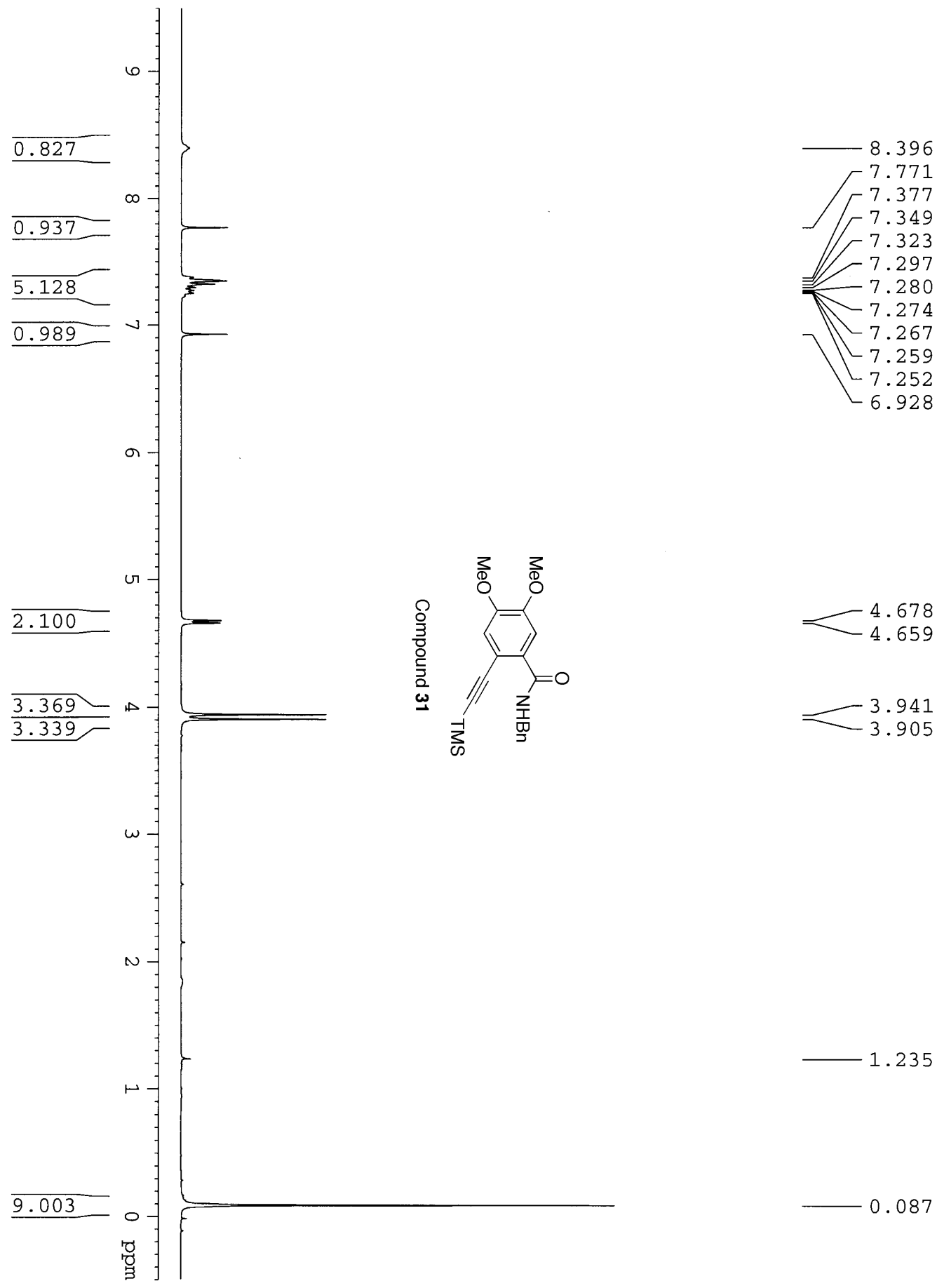

$-3.941$

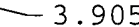

$-1.235$

$-0.087$ 

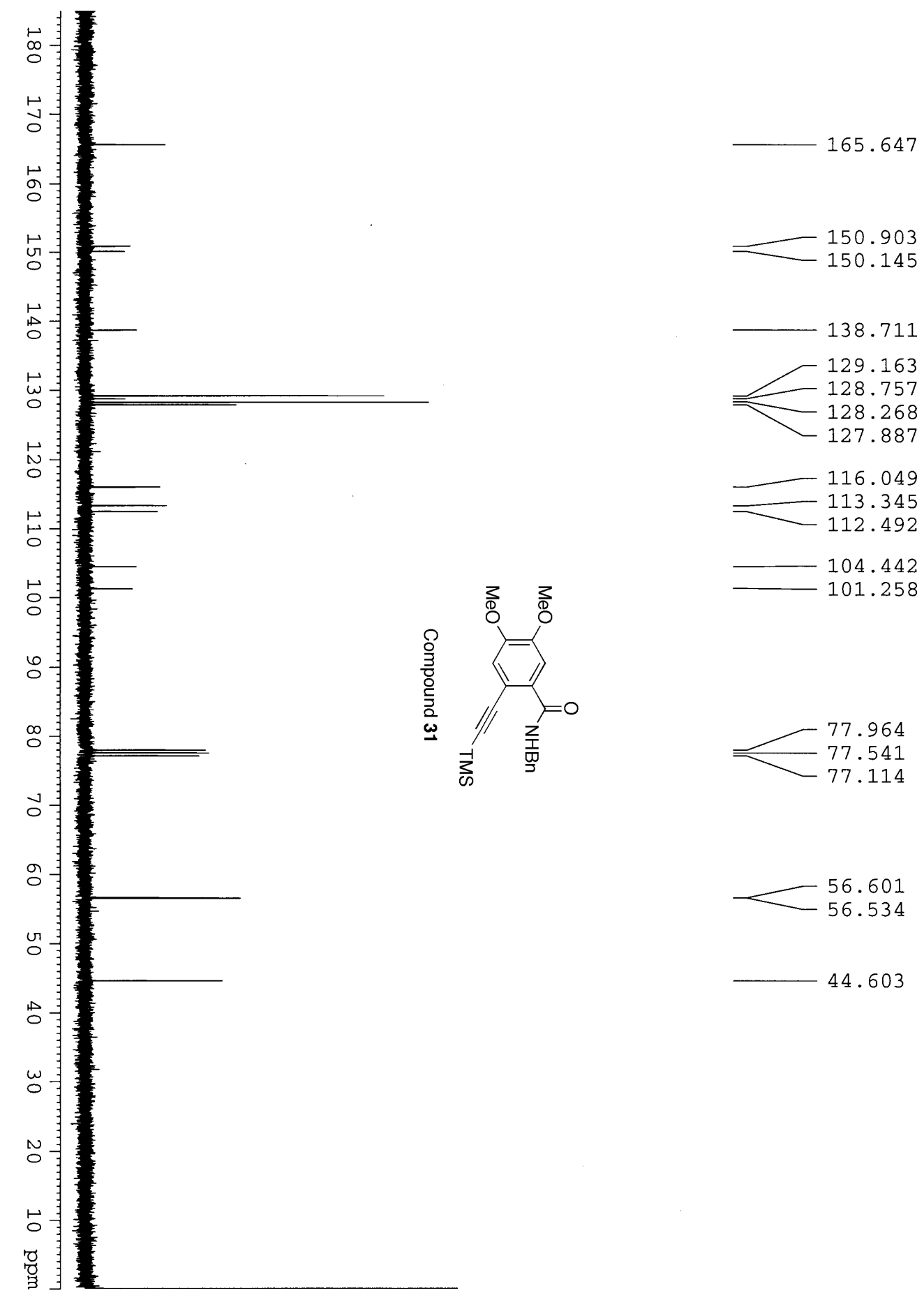

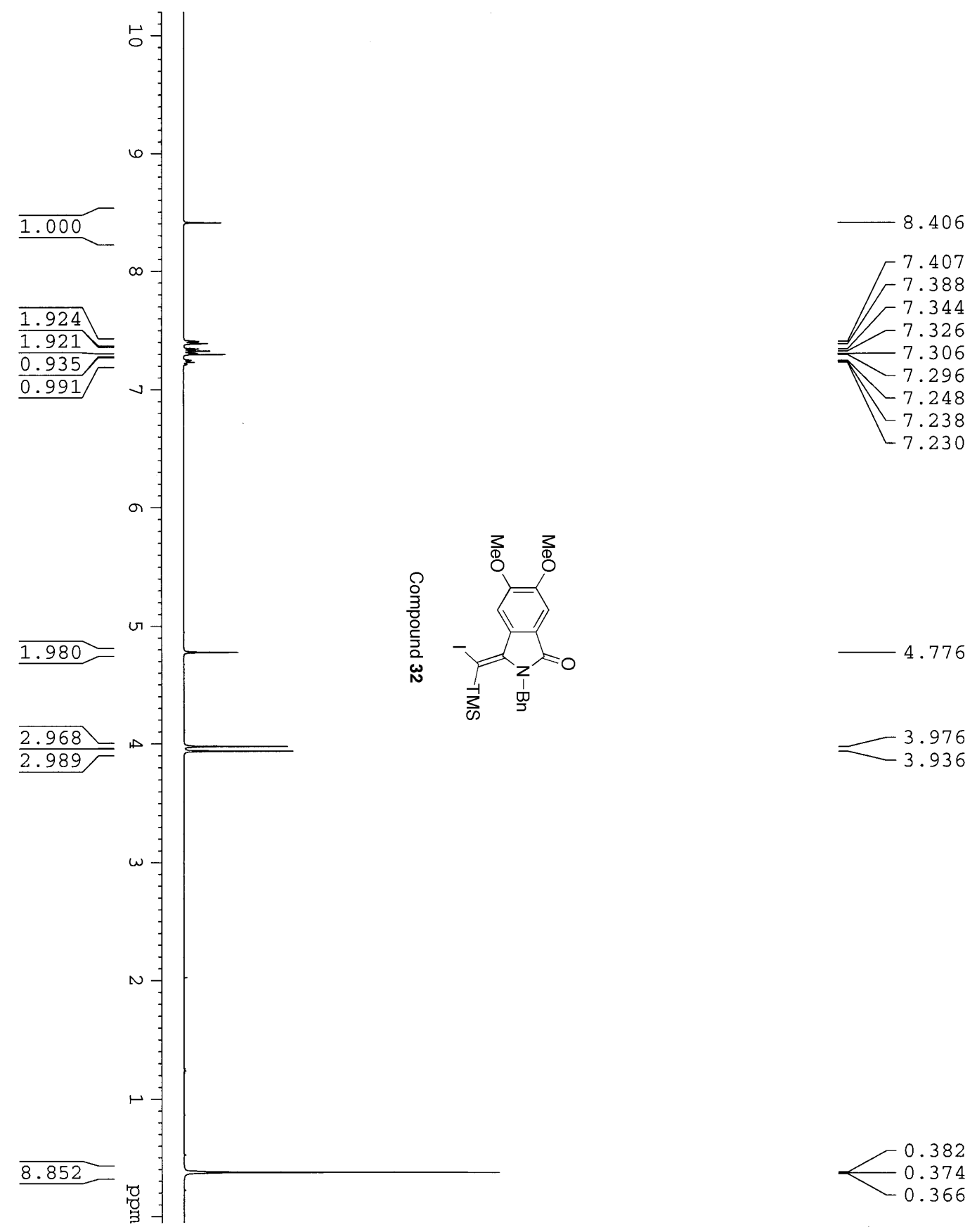

$-3.976$

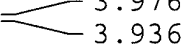

0.382

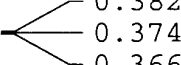



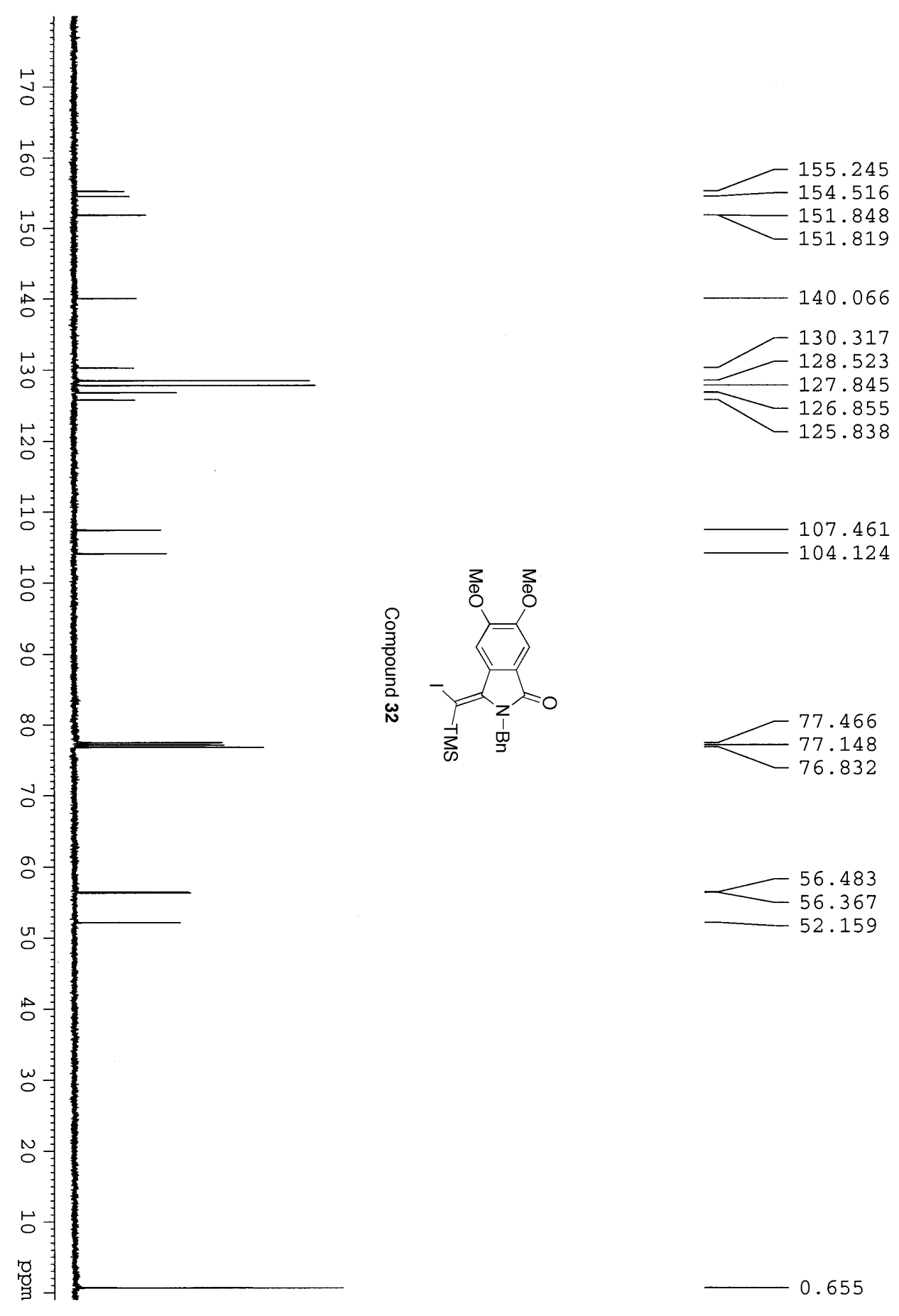

0.655 

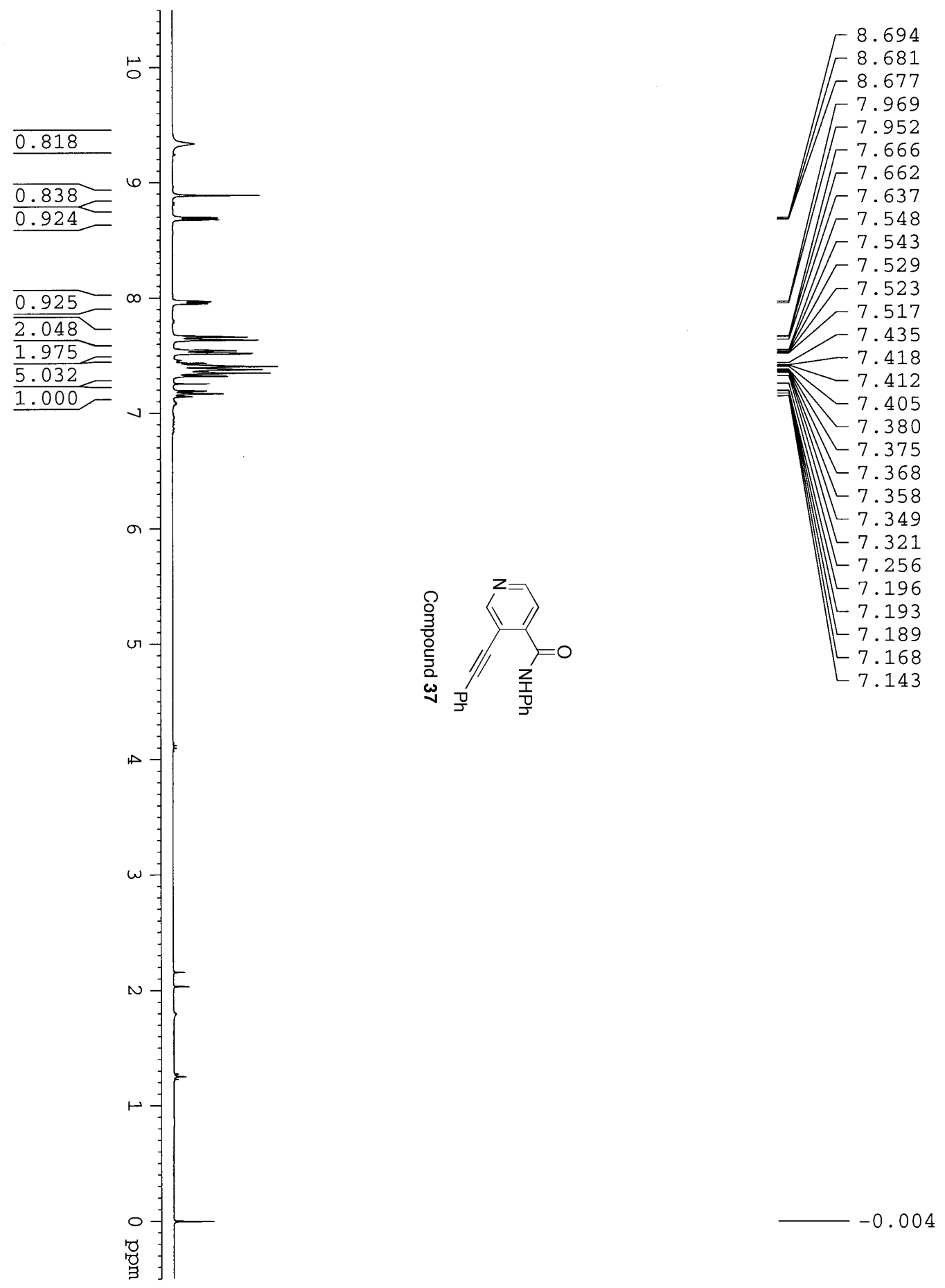

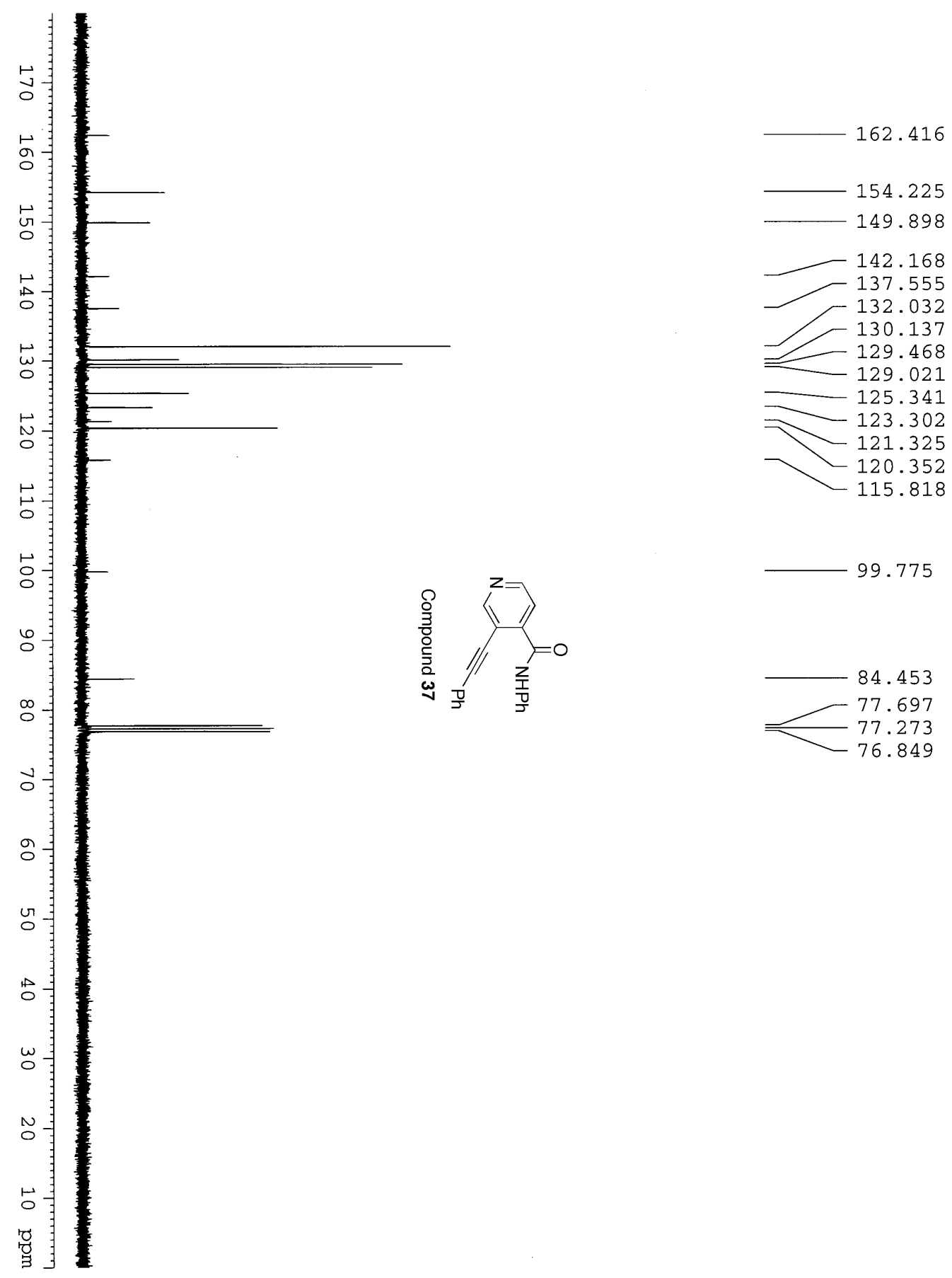

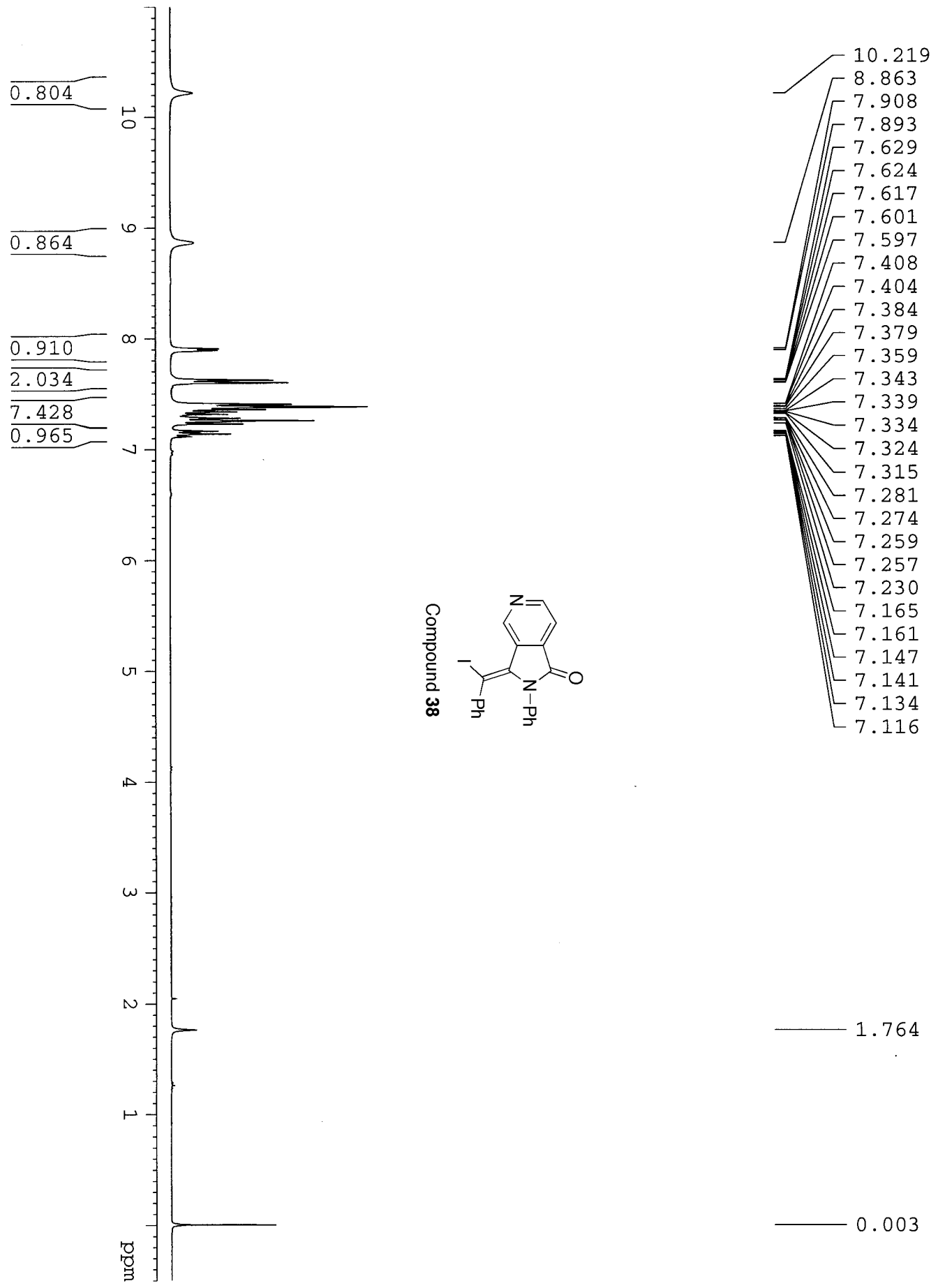


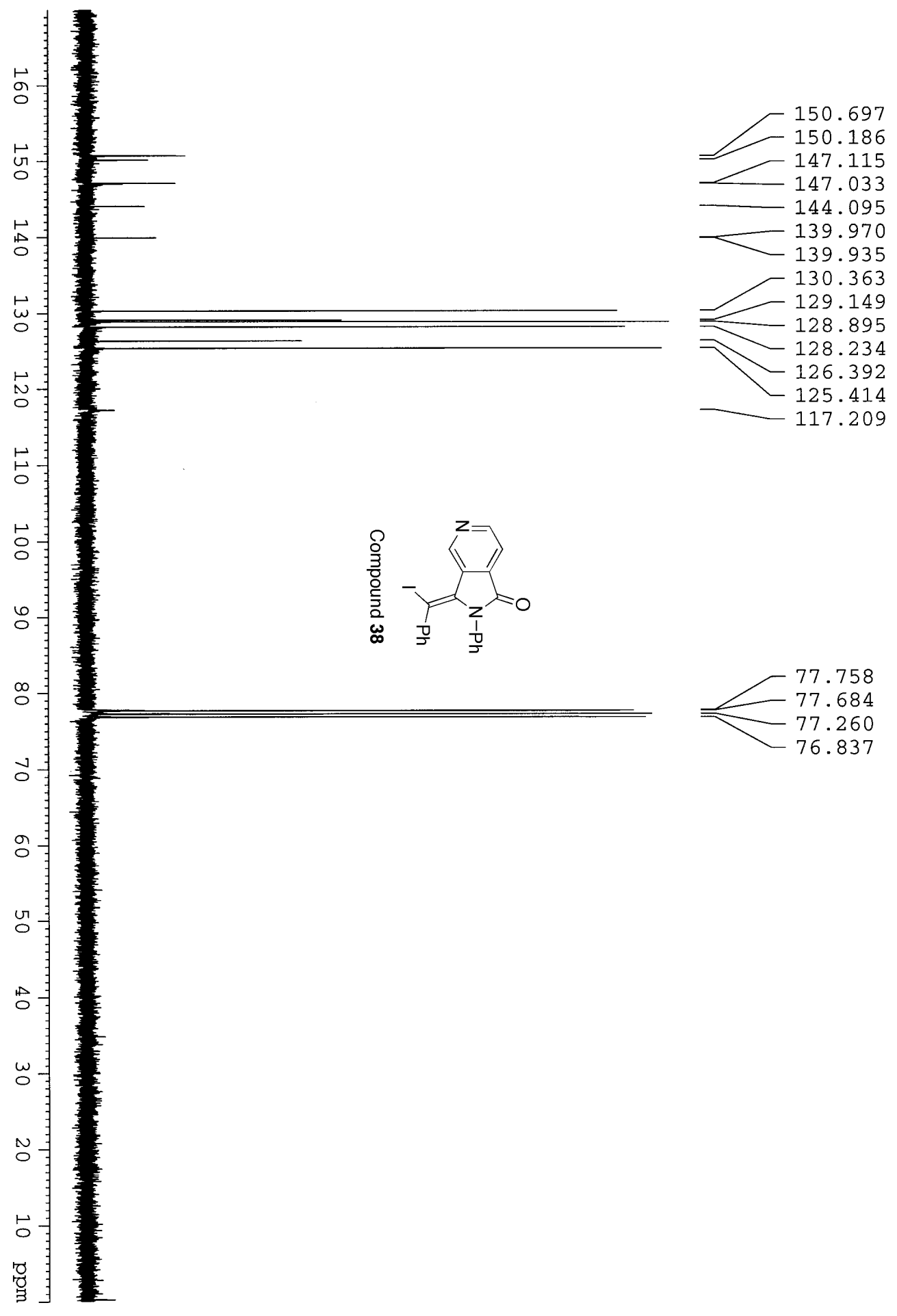



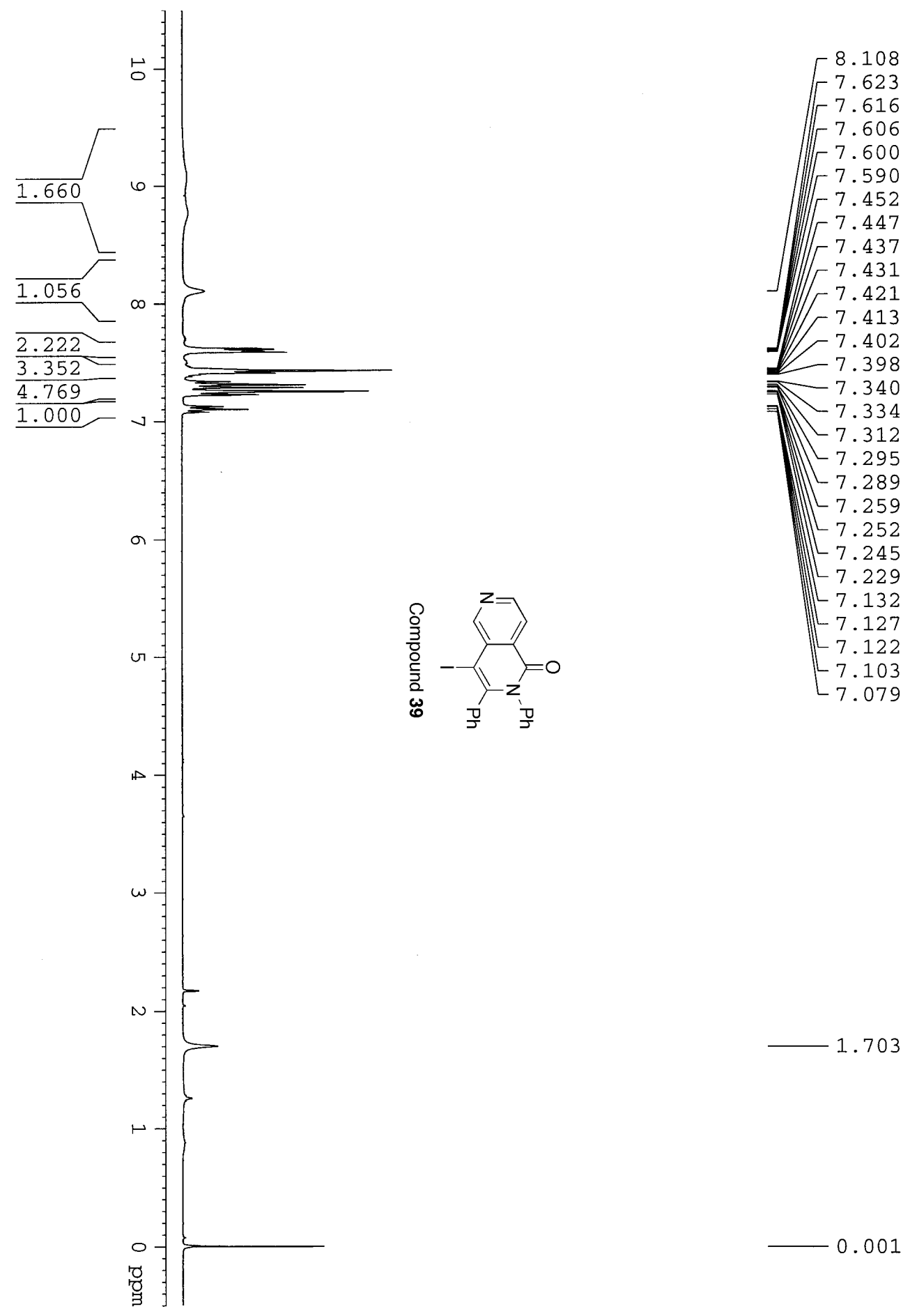

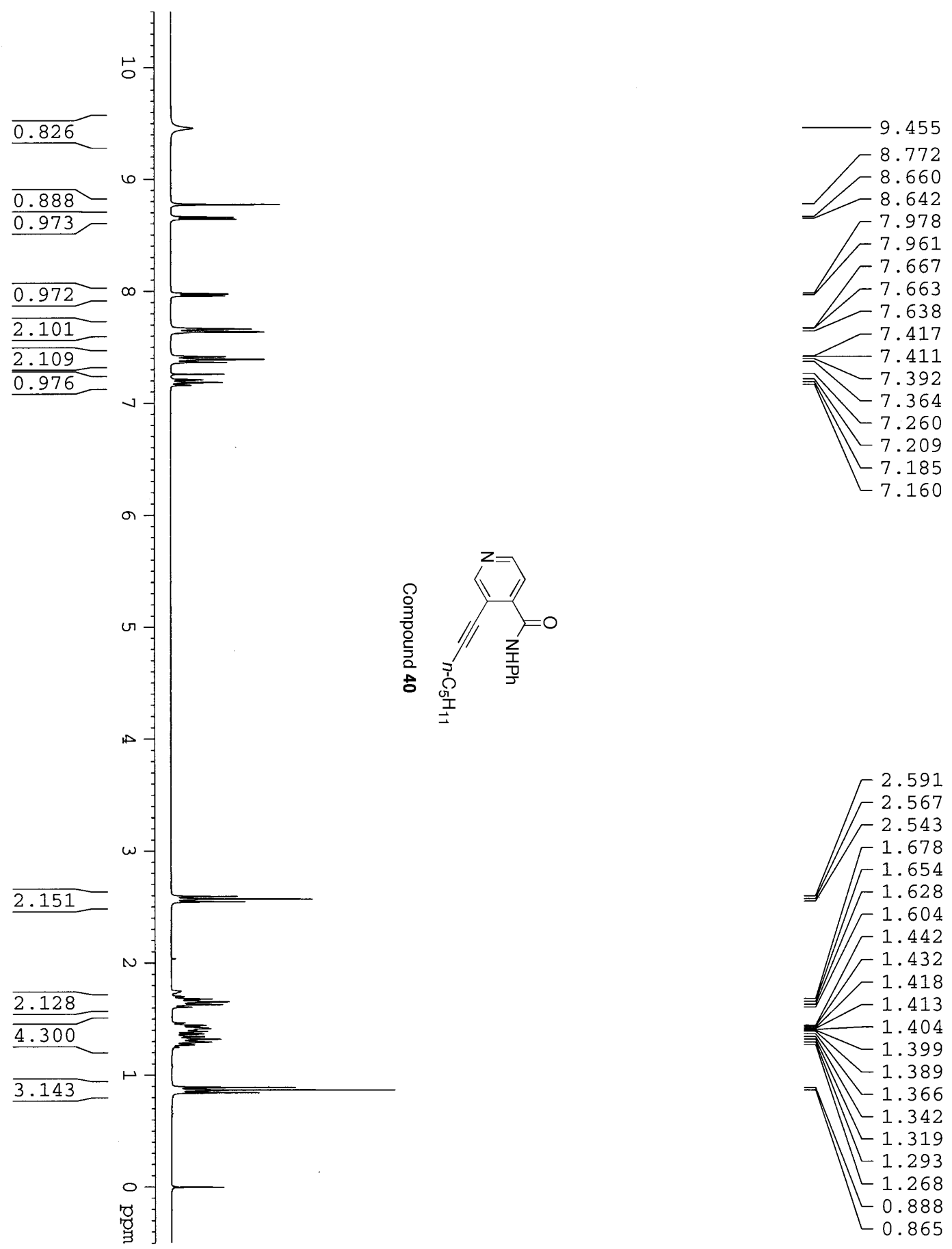

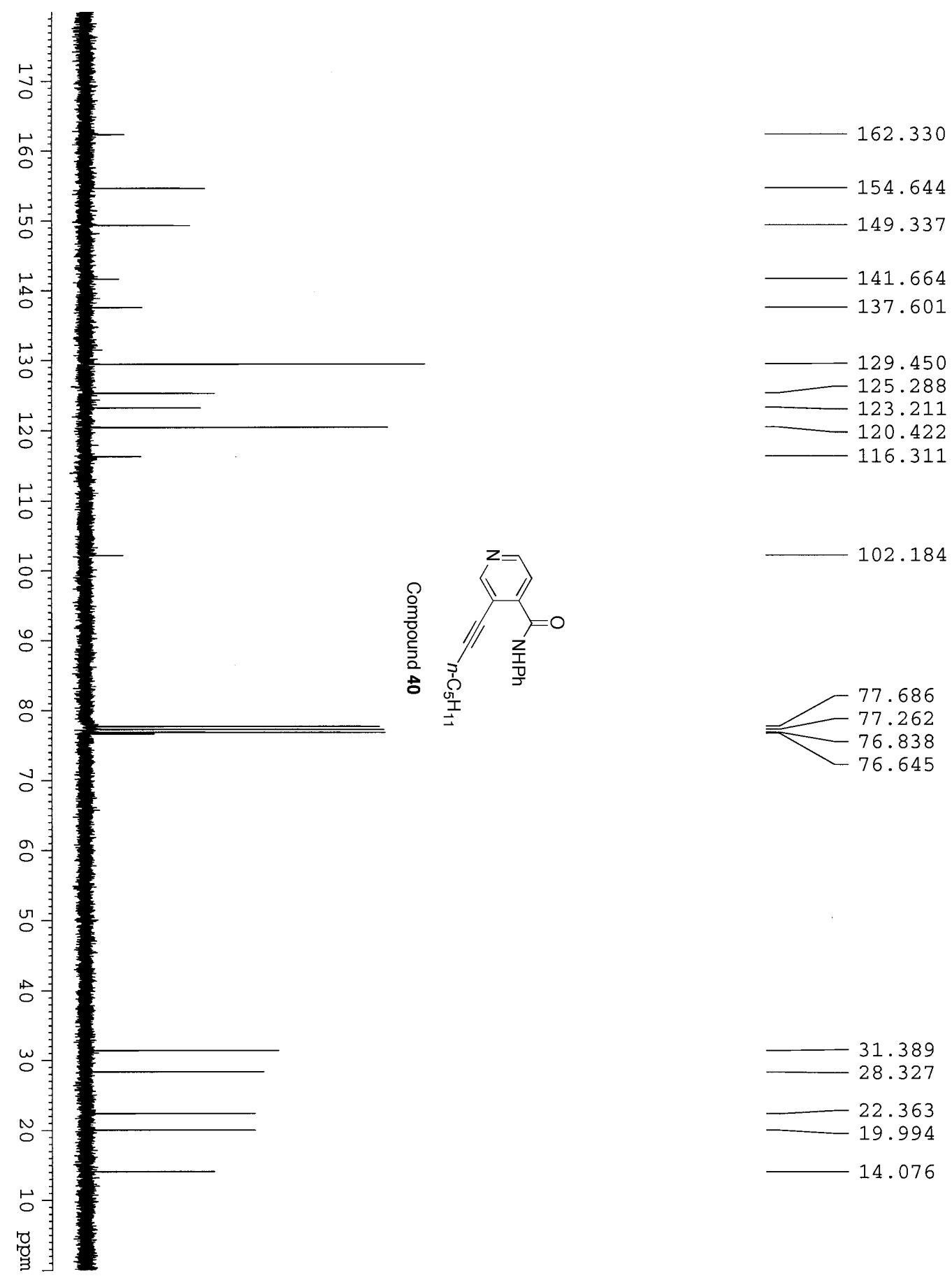

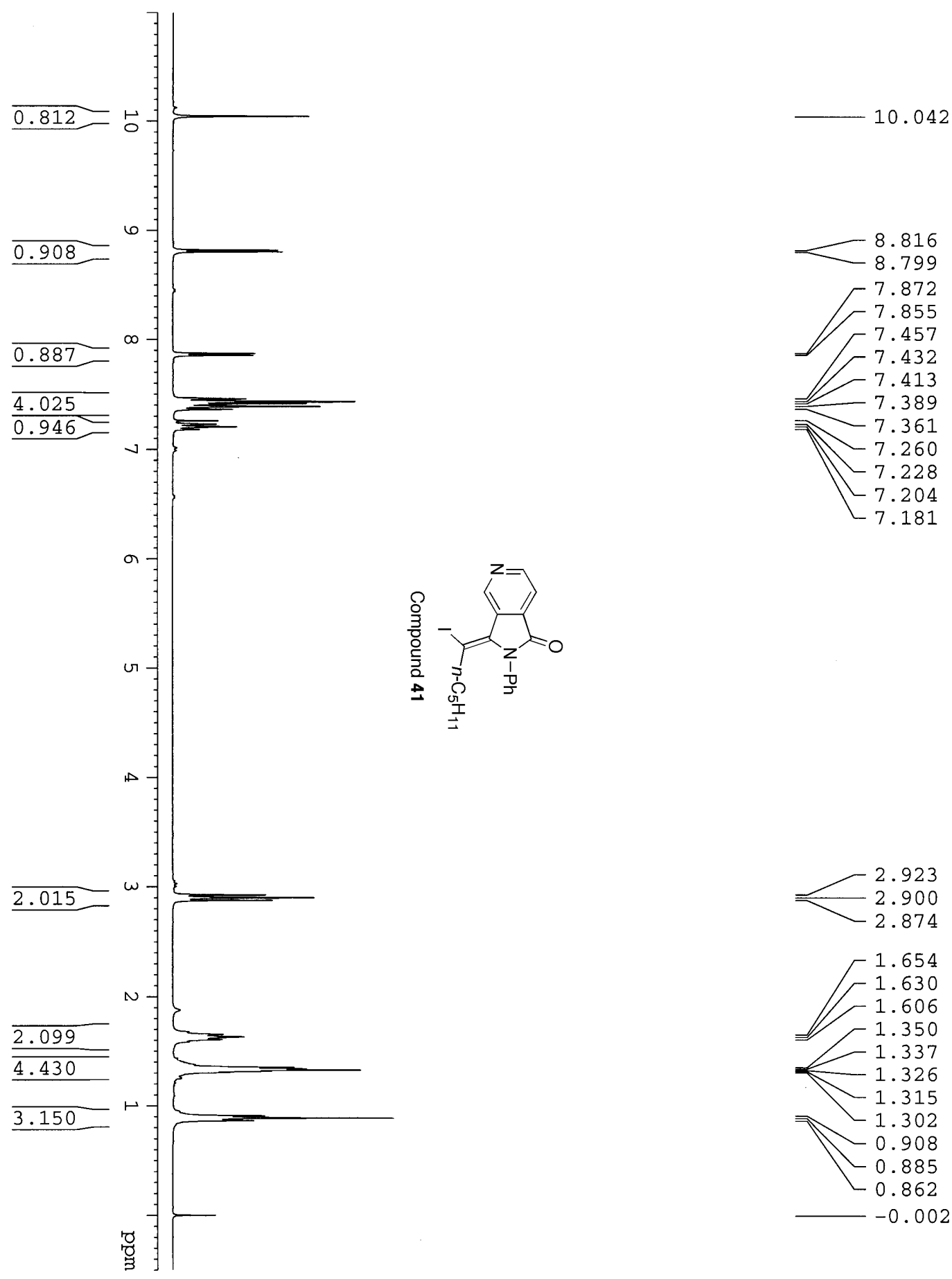

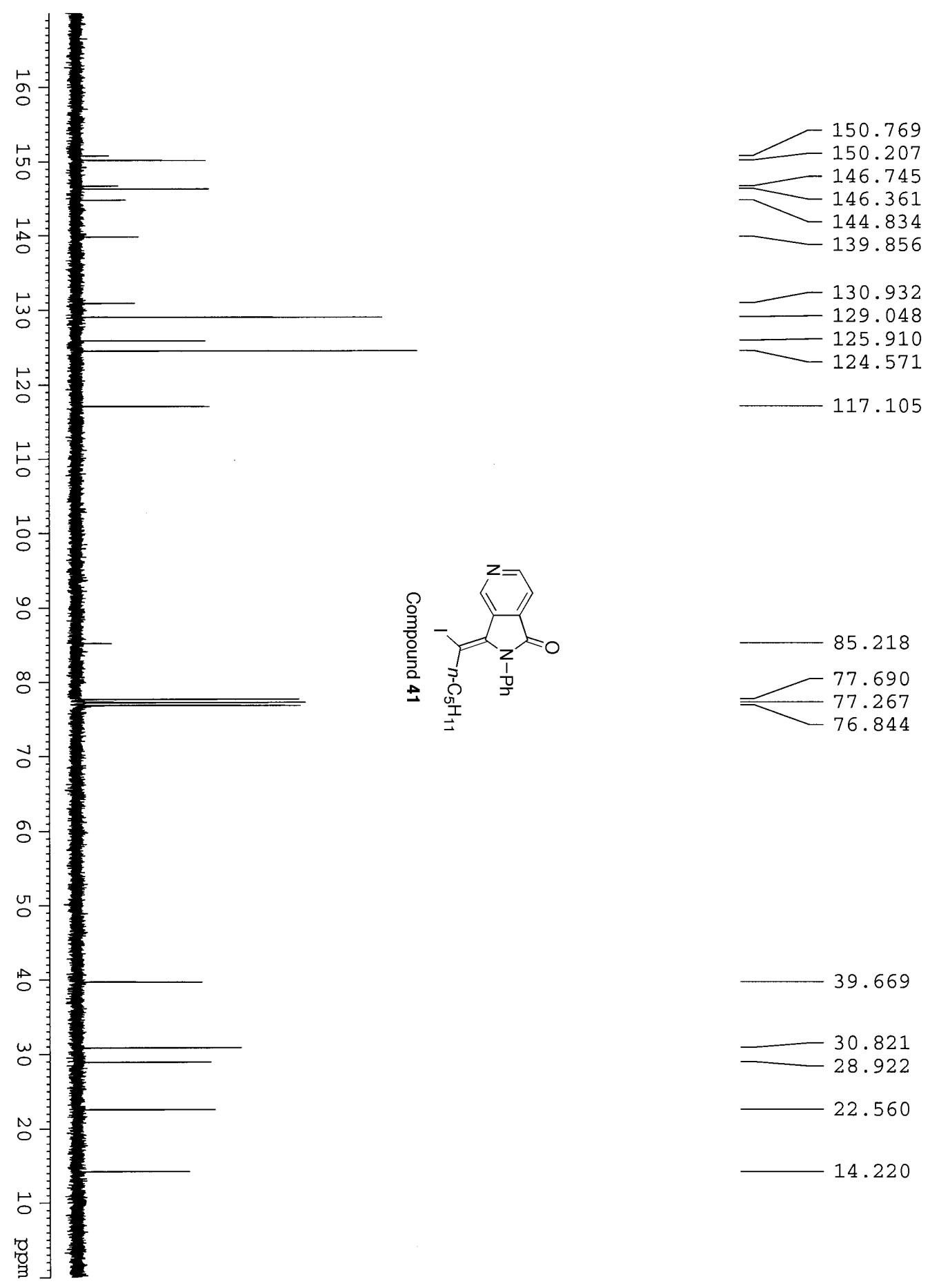

39.669

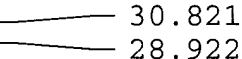

22.560

14.220 

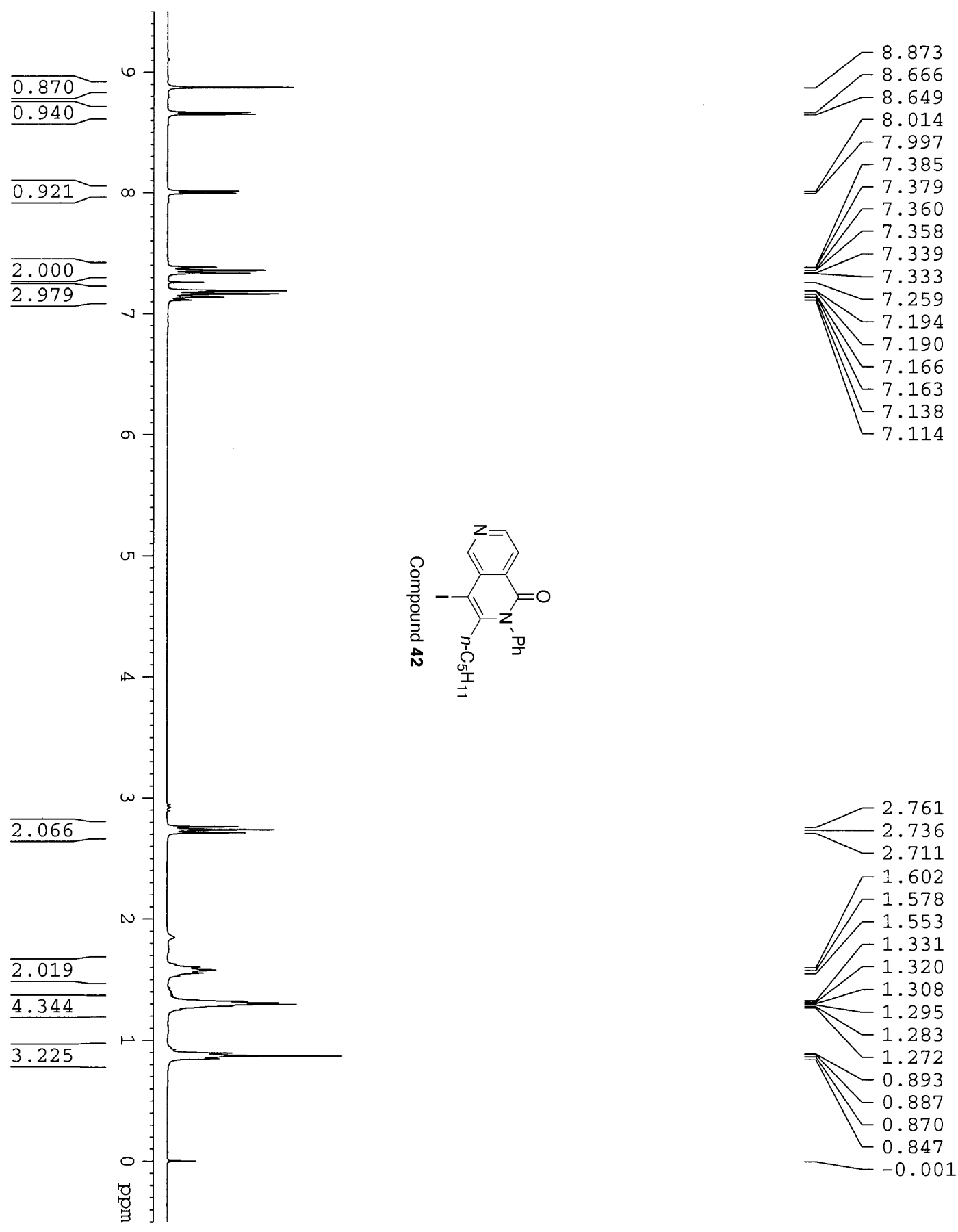

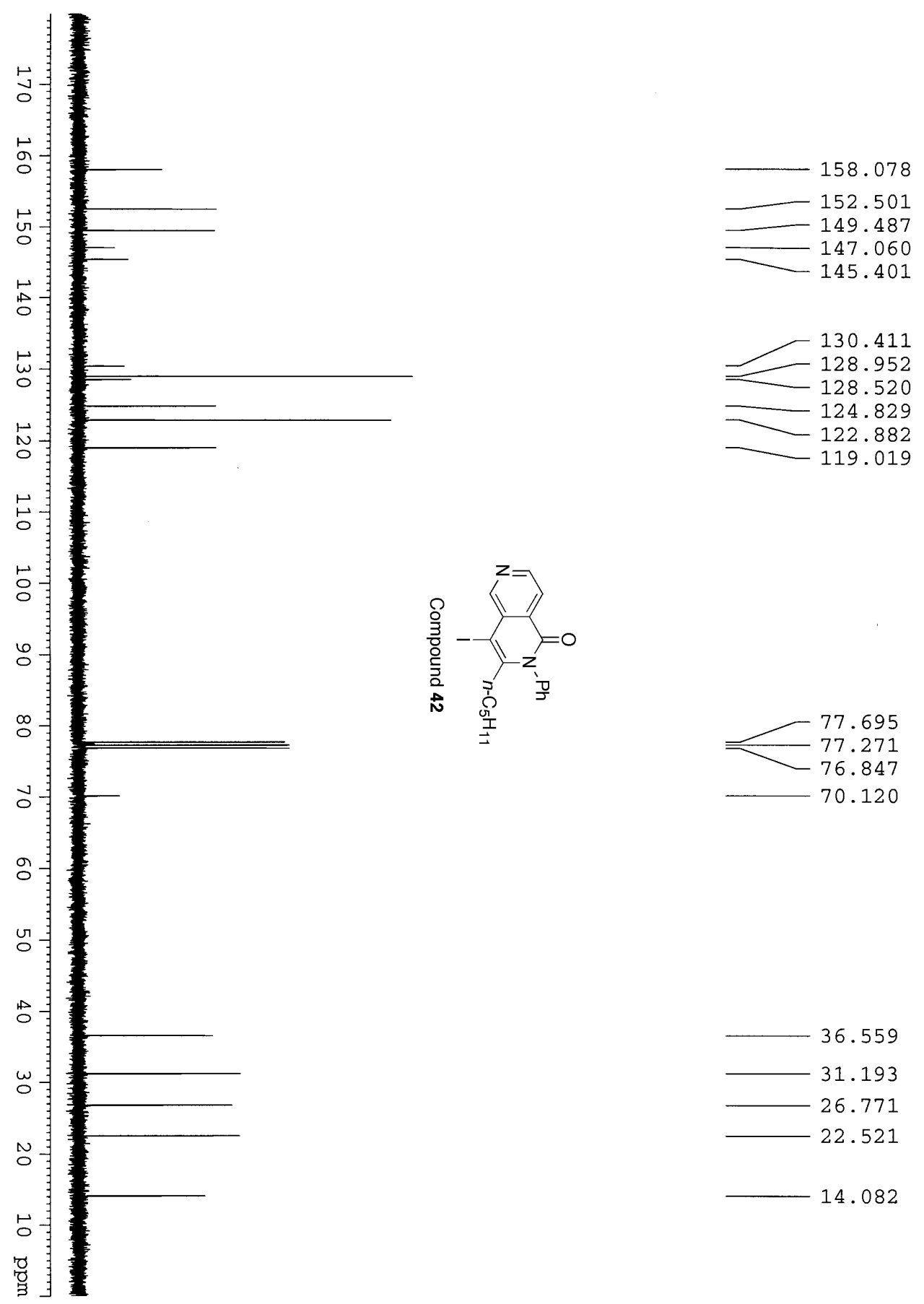

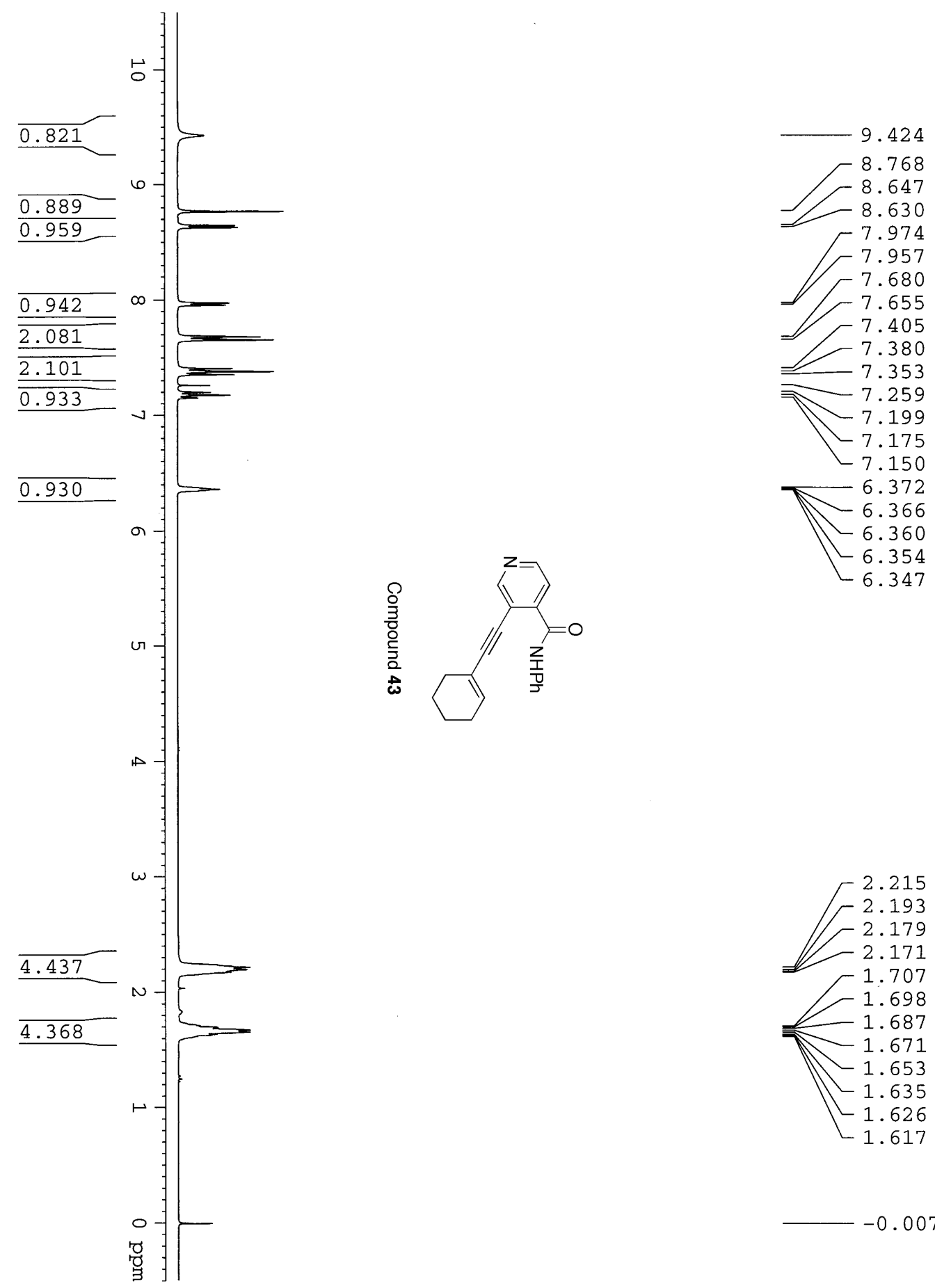

ᄂ 1.617

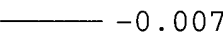




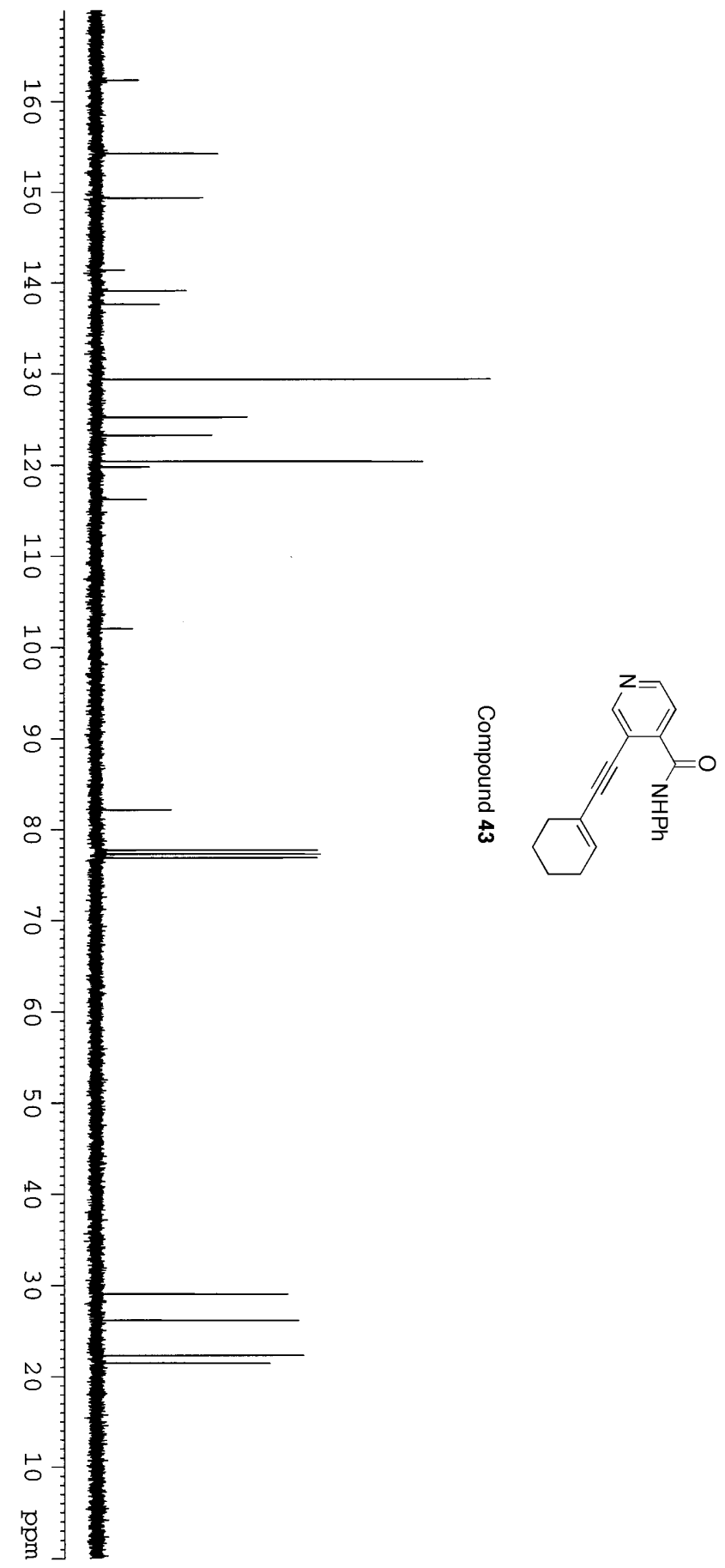

162.323

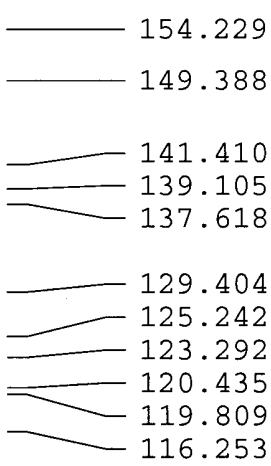

102.061
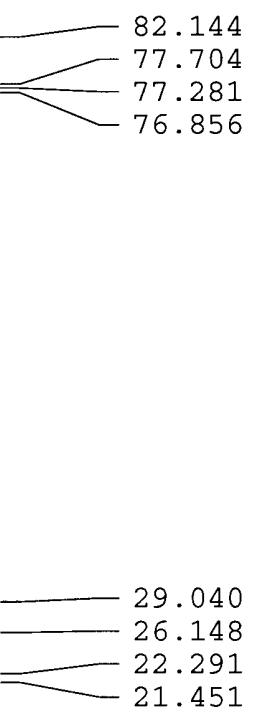

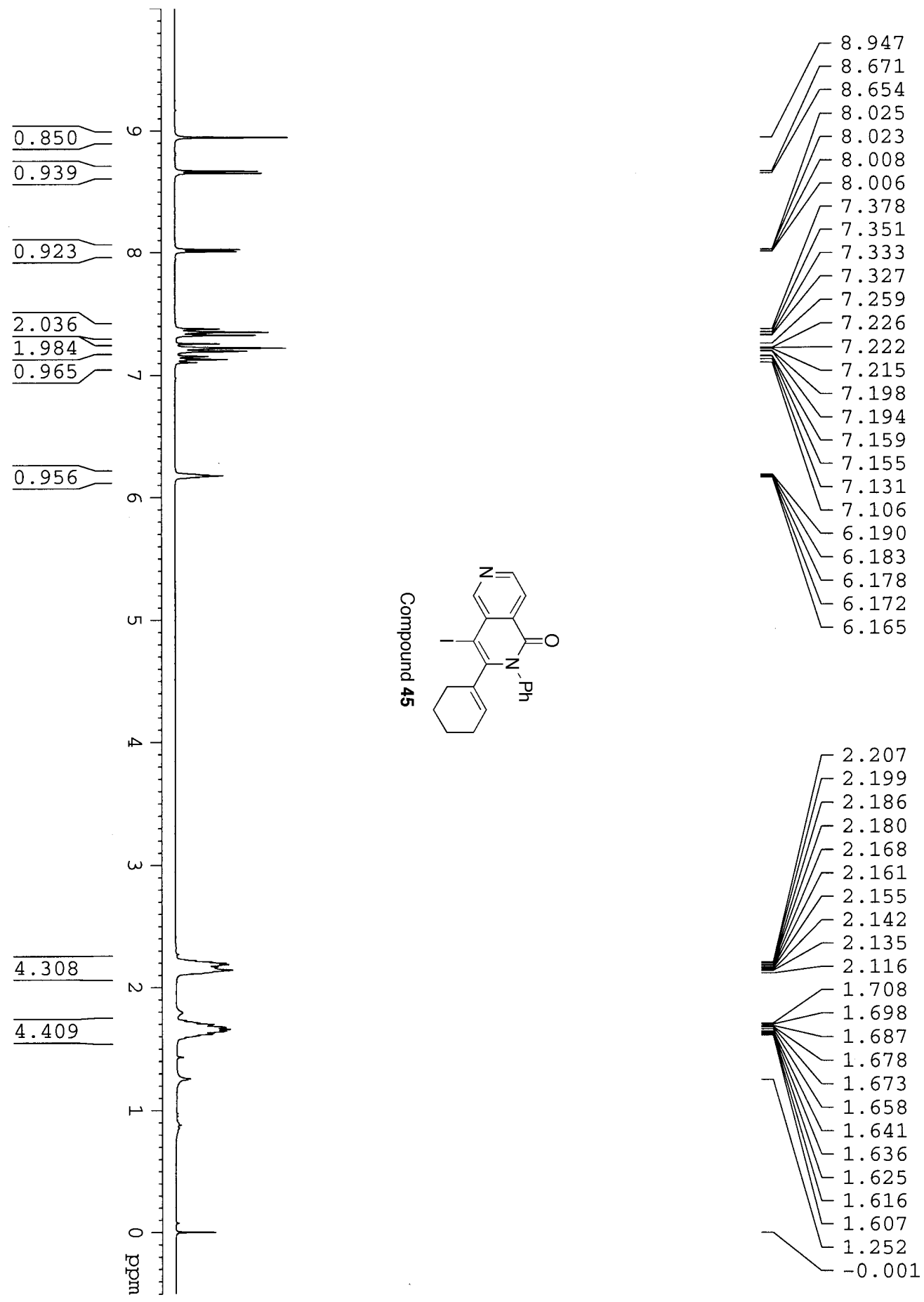

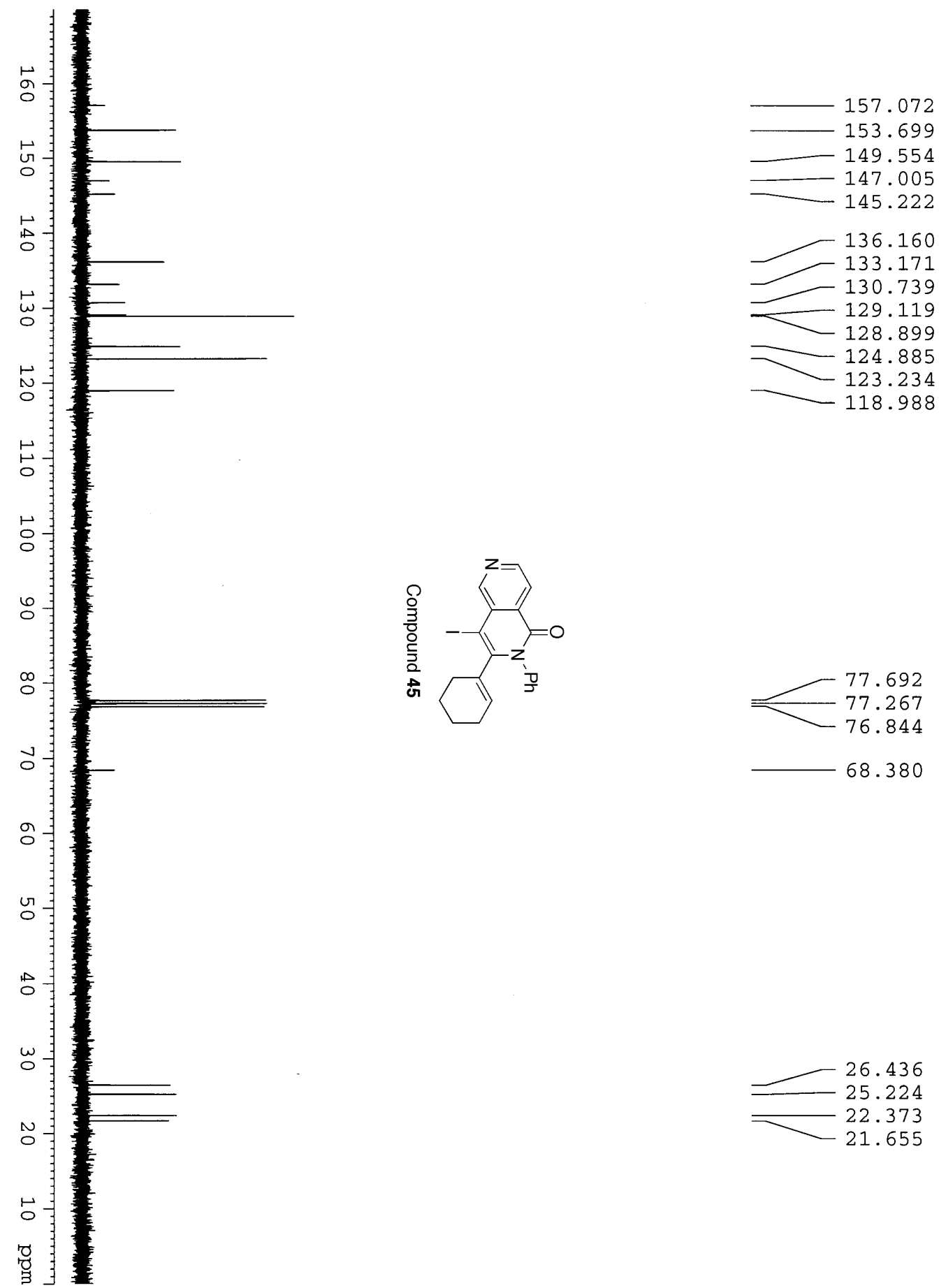

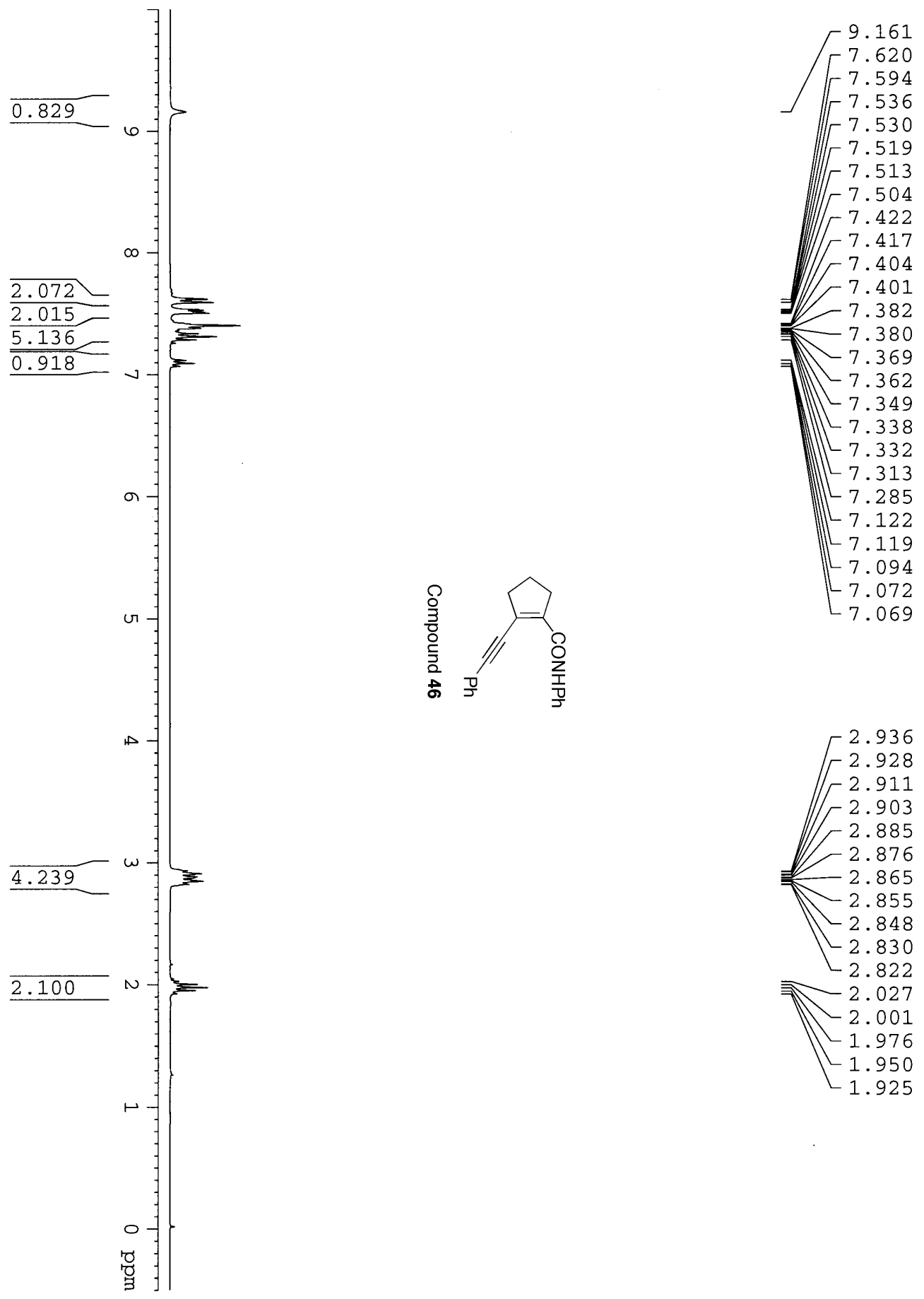

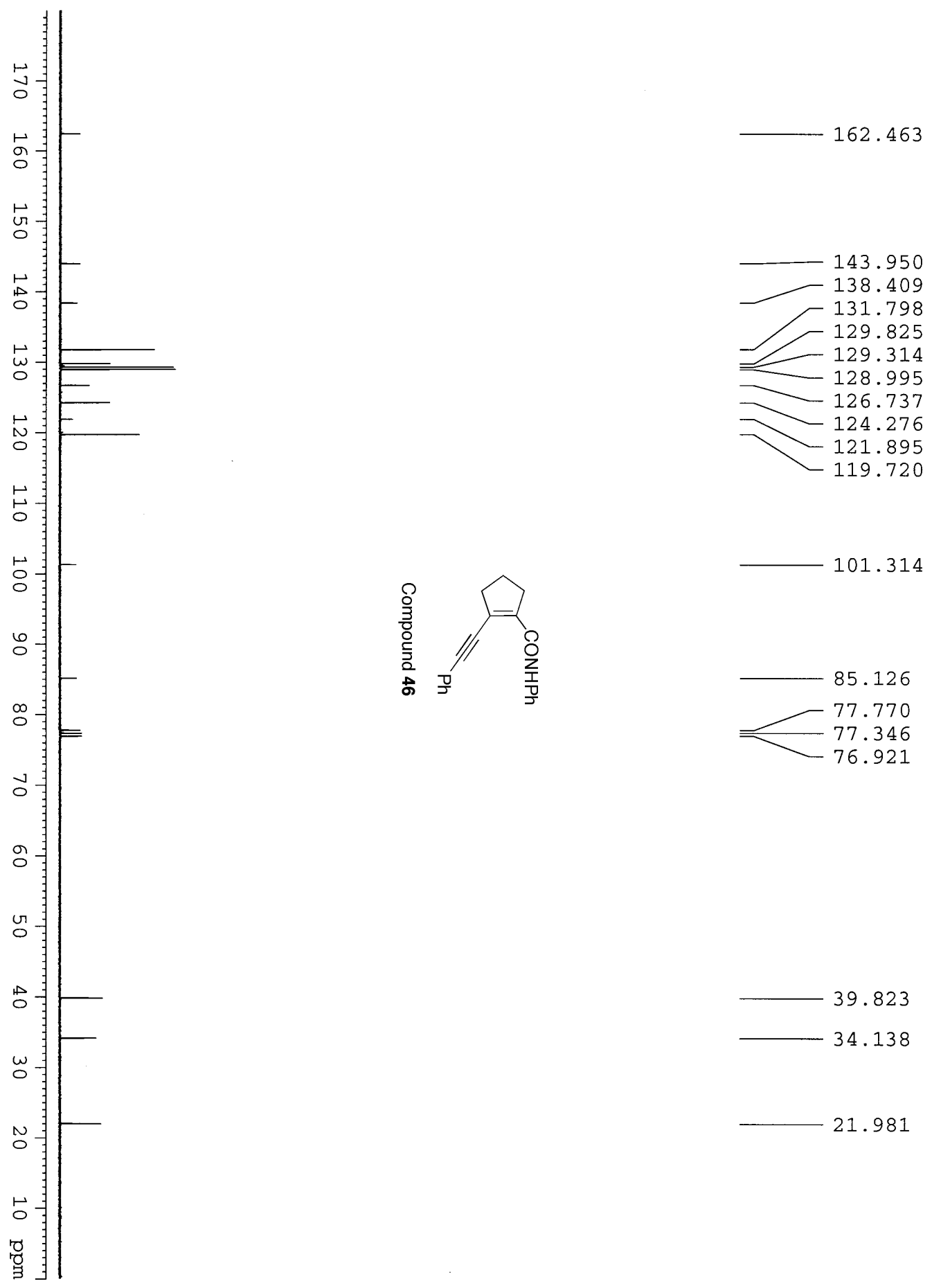

39.823

34.138

21.981 

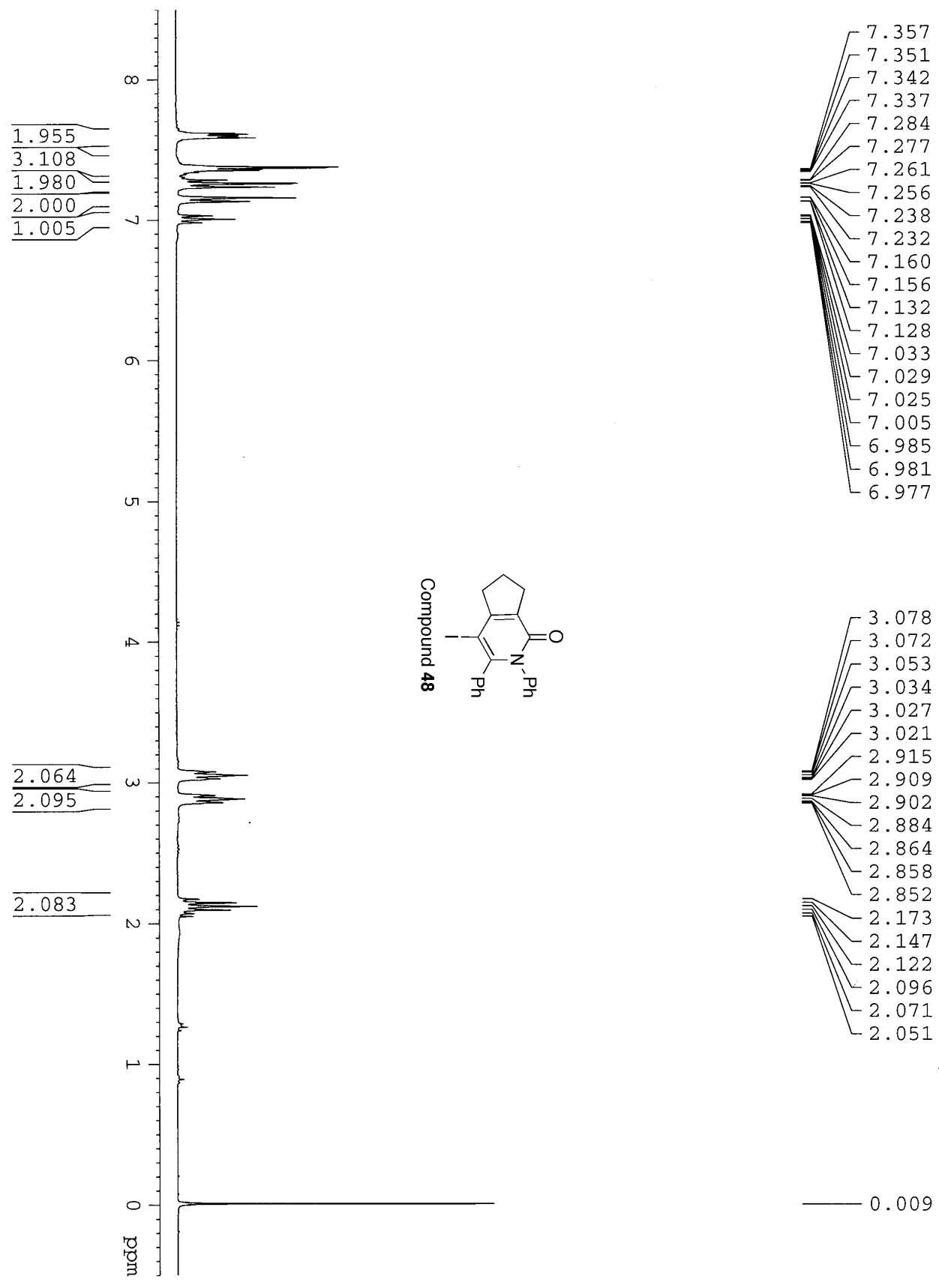

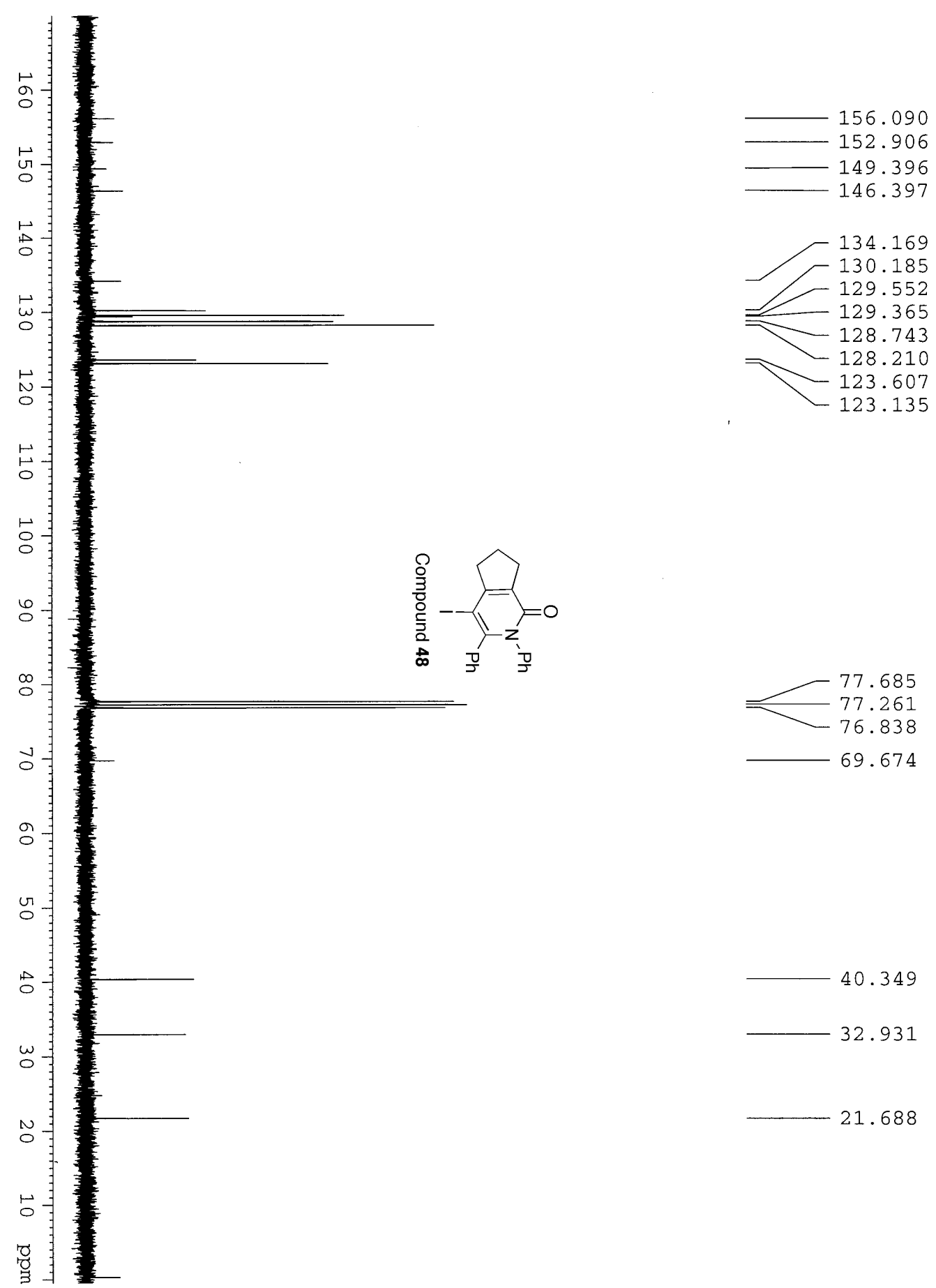

40.349

32.931

21.688 

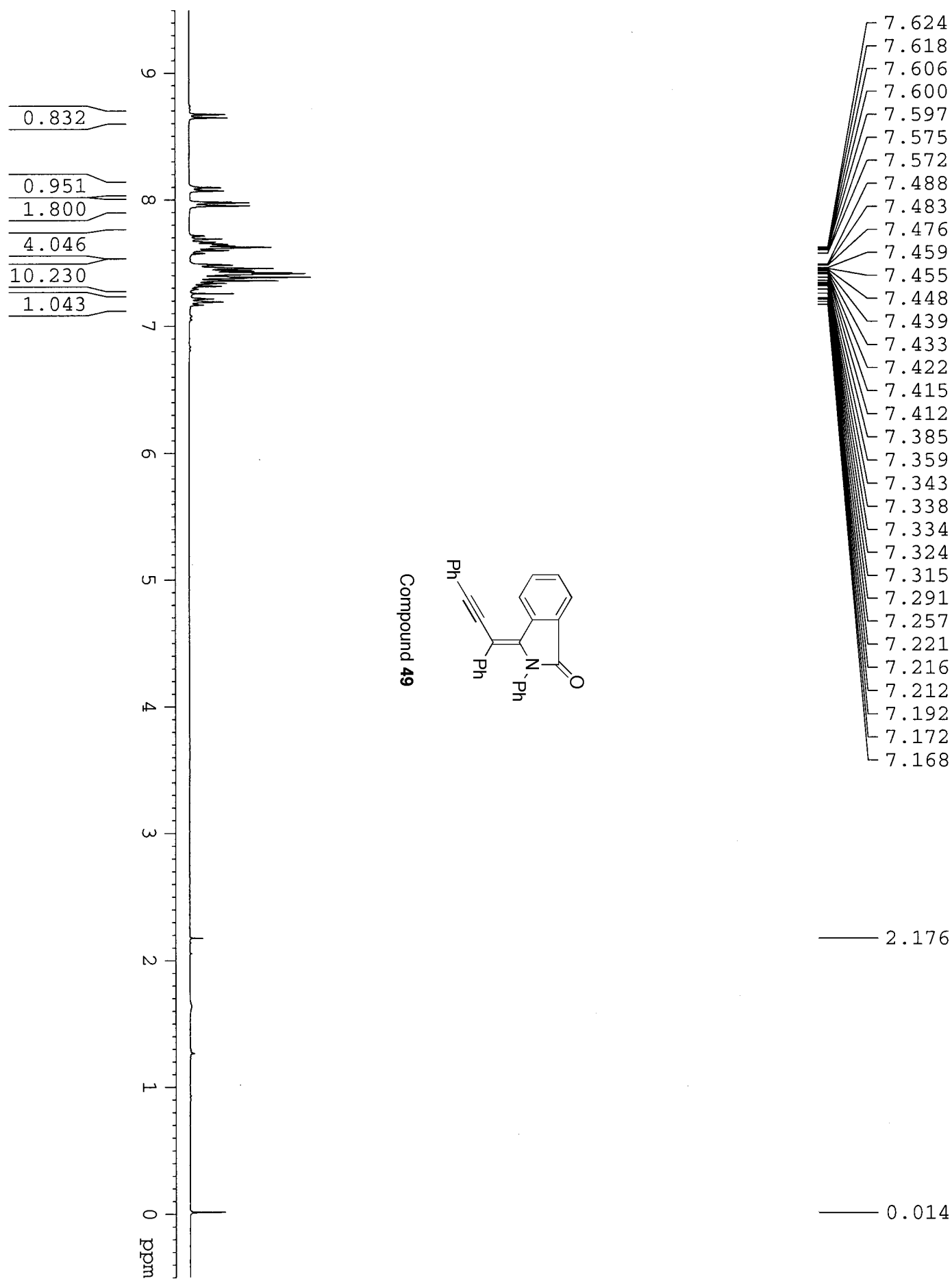

$-0.014$ 


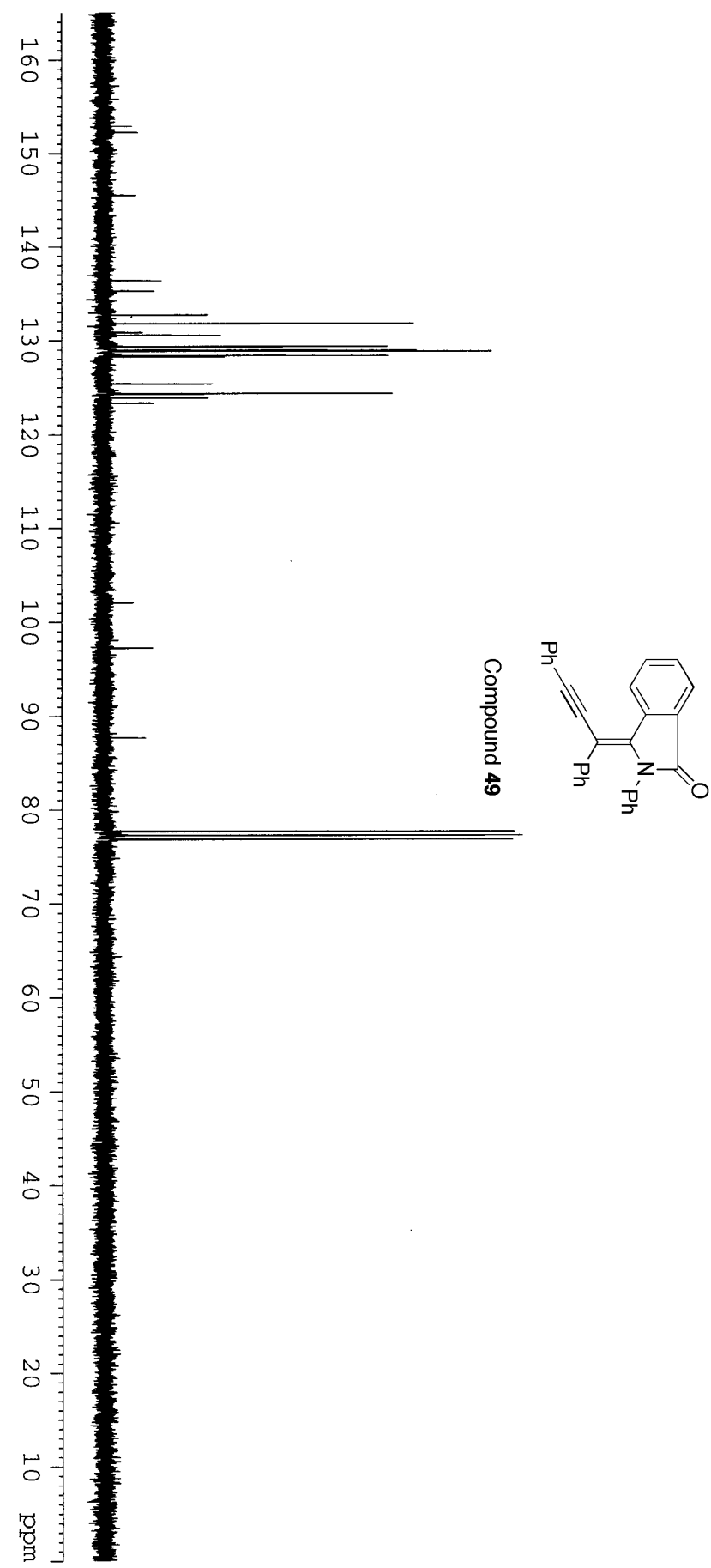



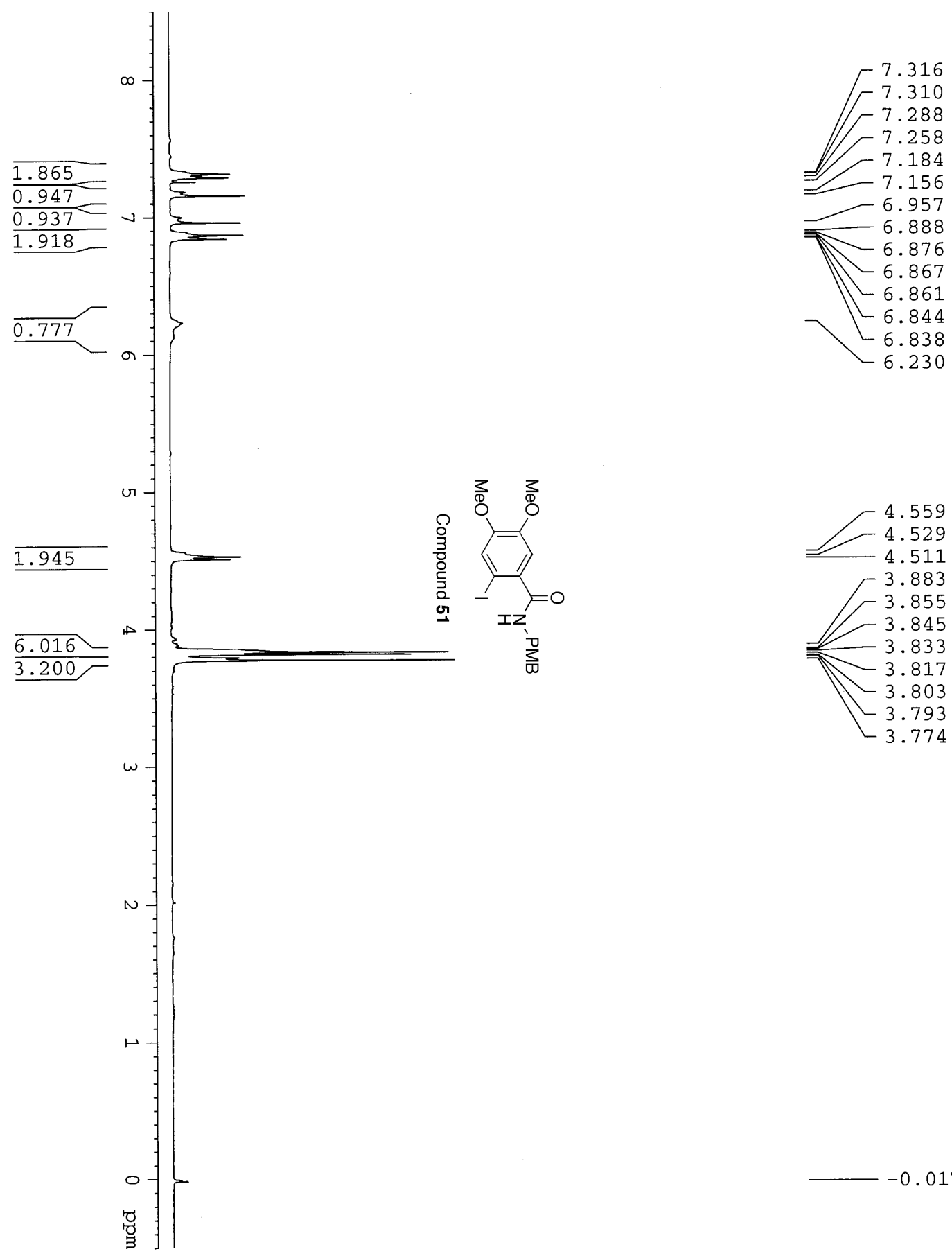

$-0.017$ 

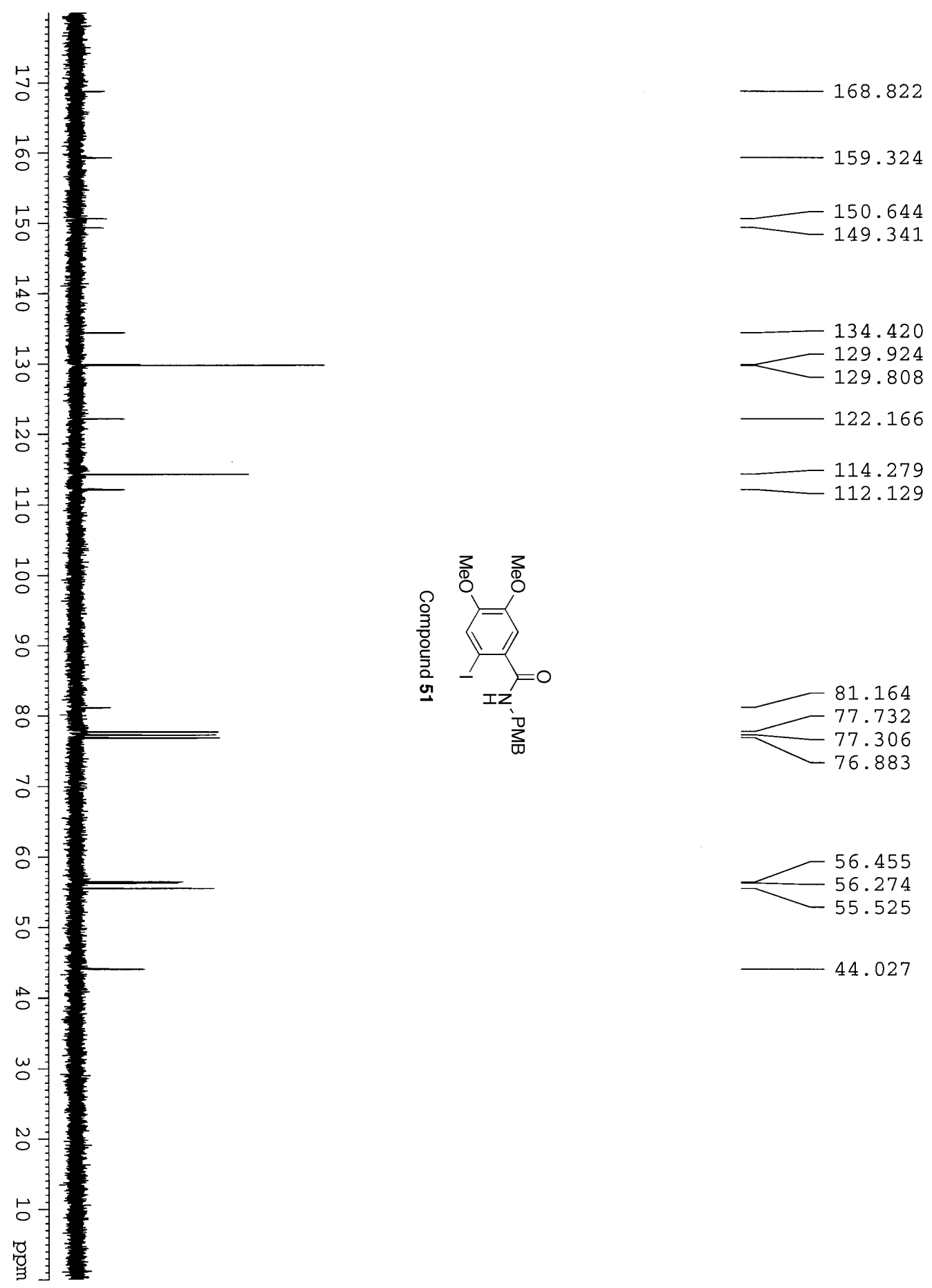
44.027 


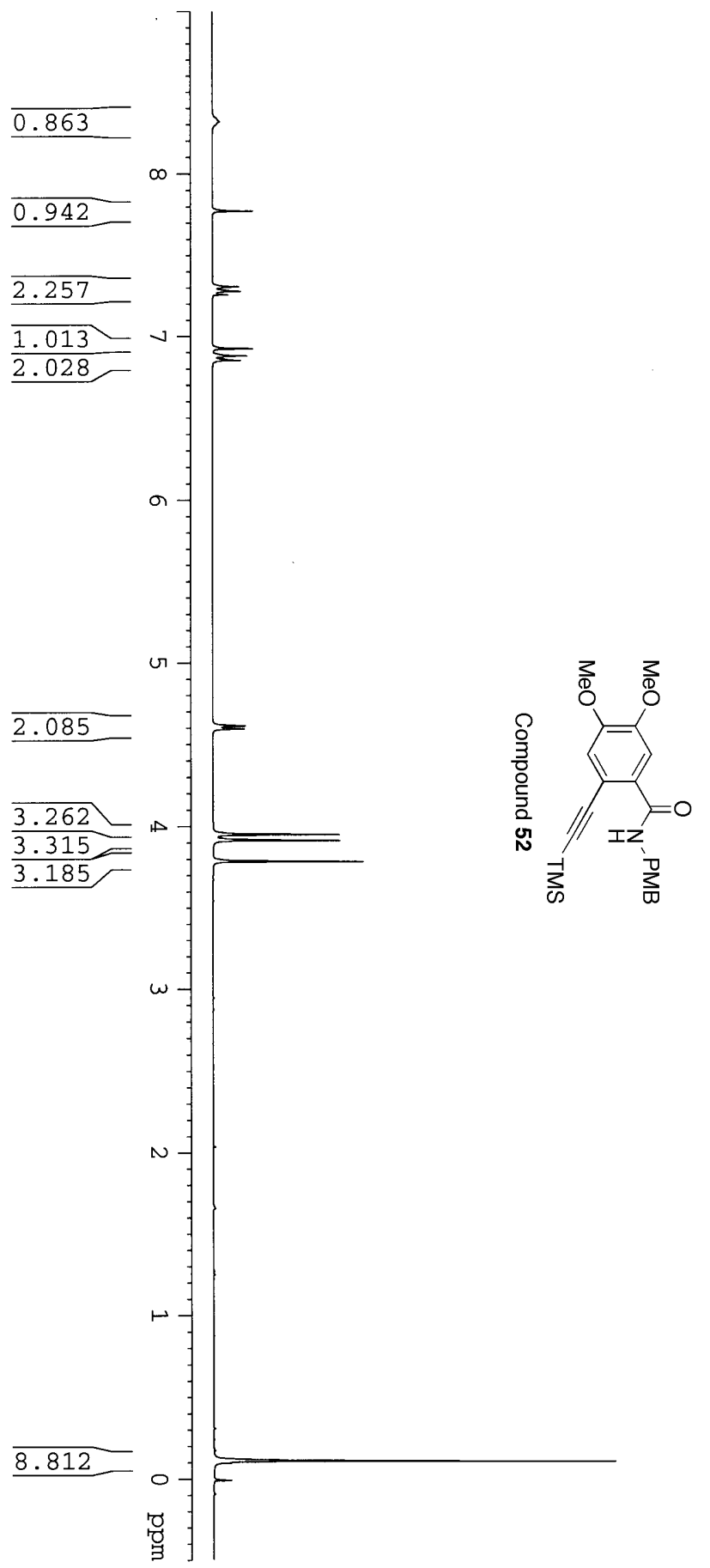
8.321

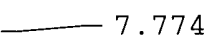

$-7.308$ $-7.280$

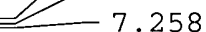

$-6.925$ $-6.881$

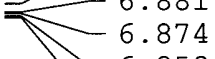

$-6.858$

$-6.852$

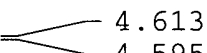

4.595
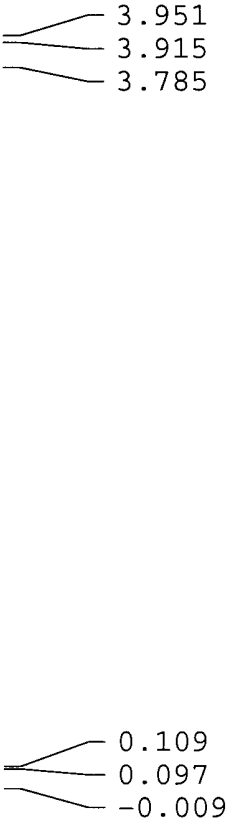

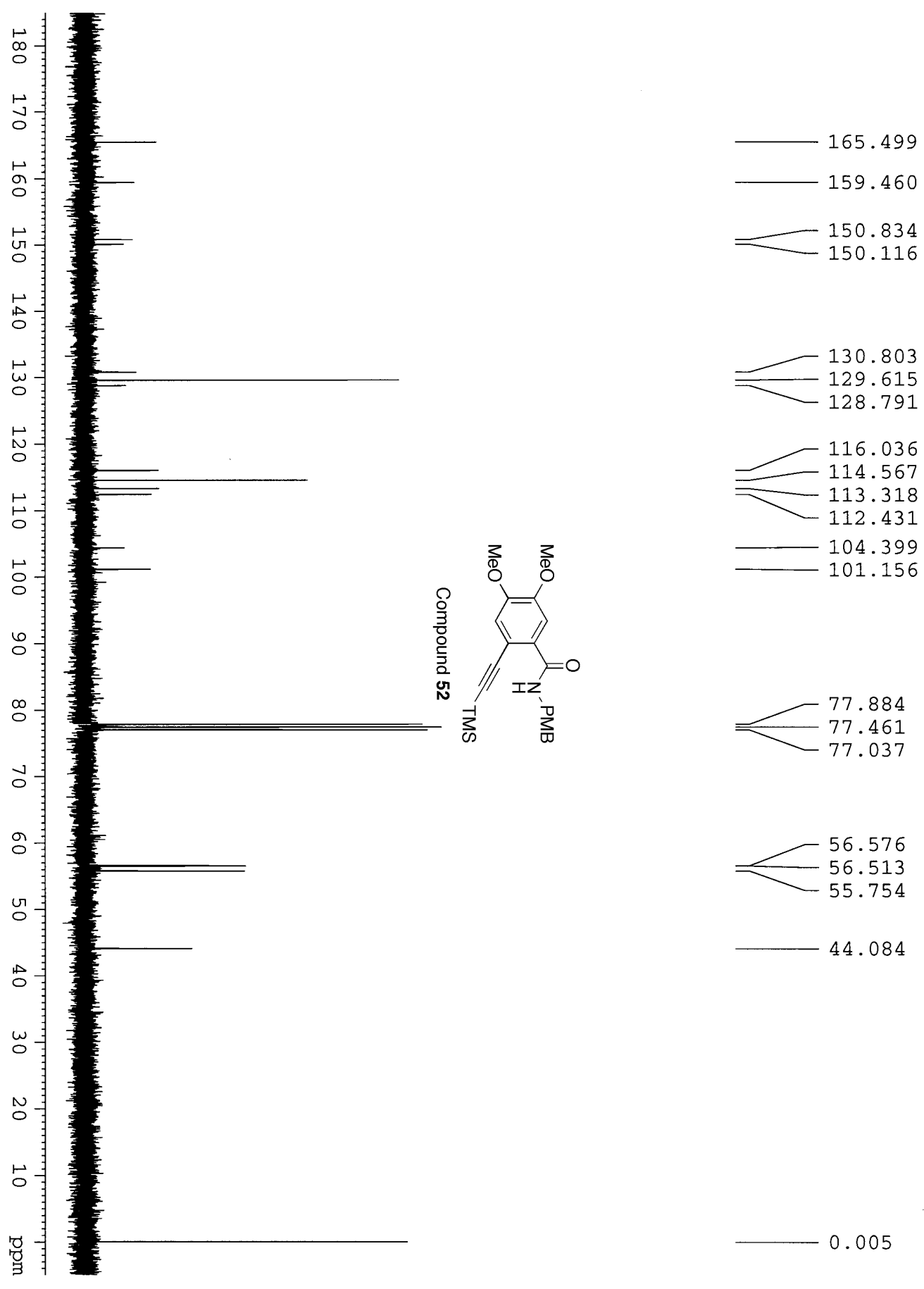

0.005 

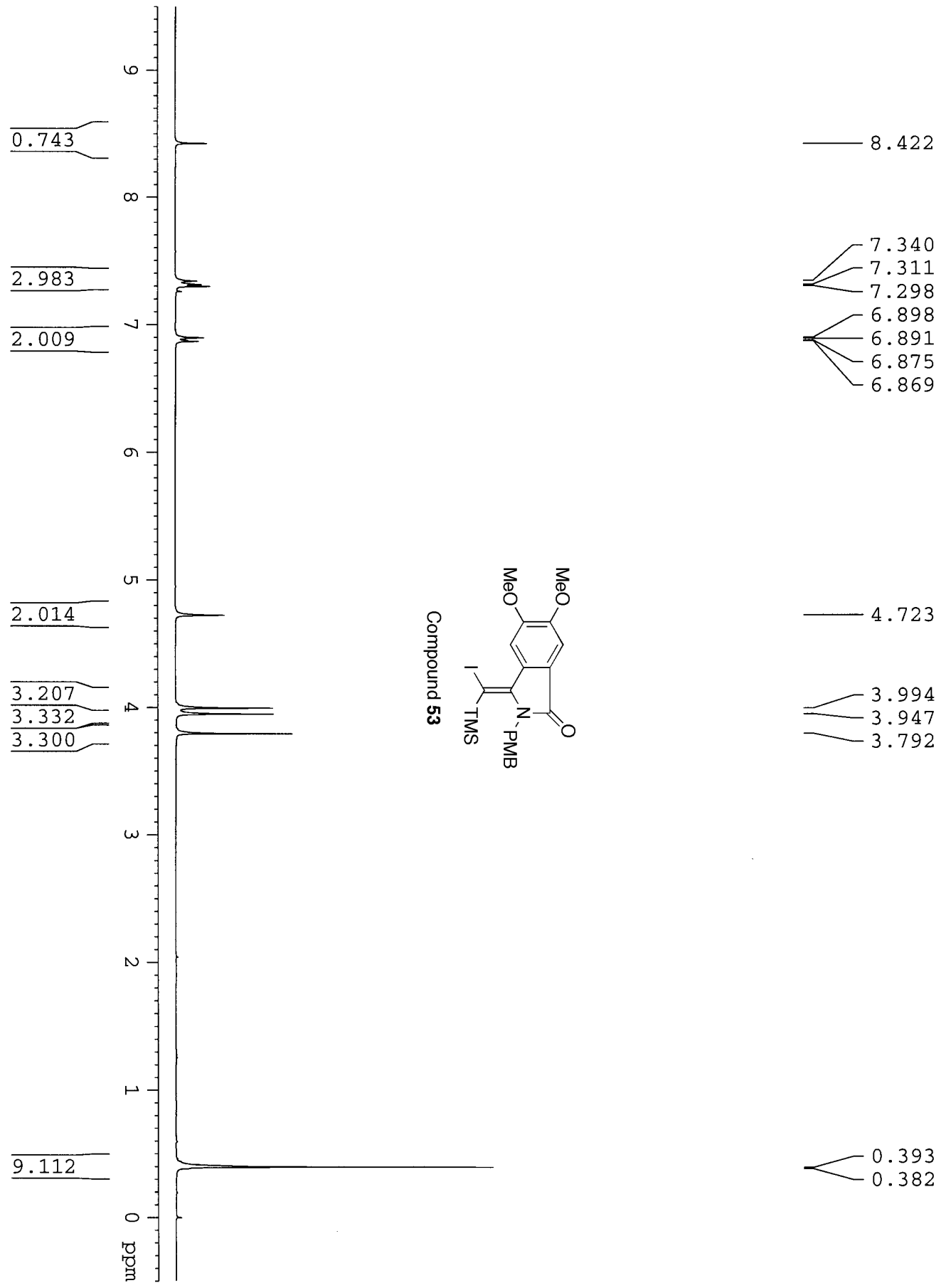

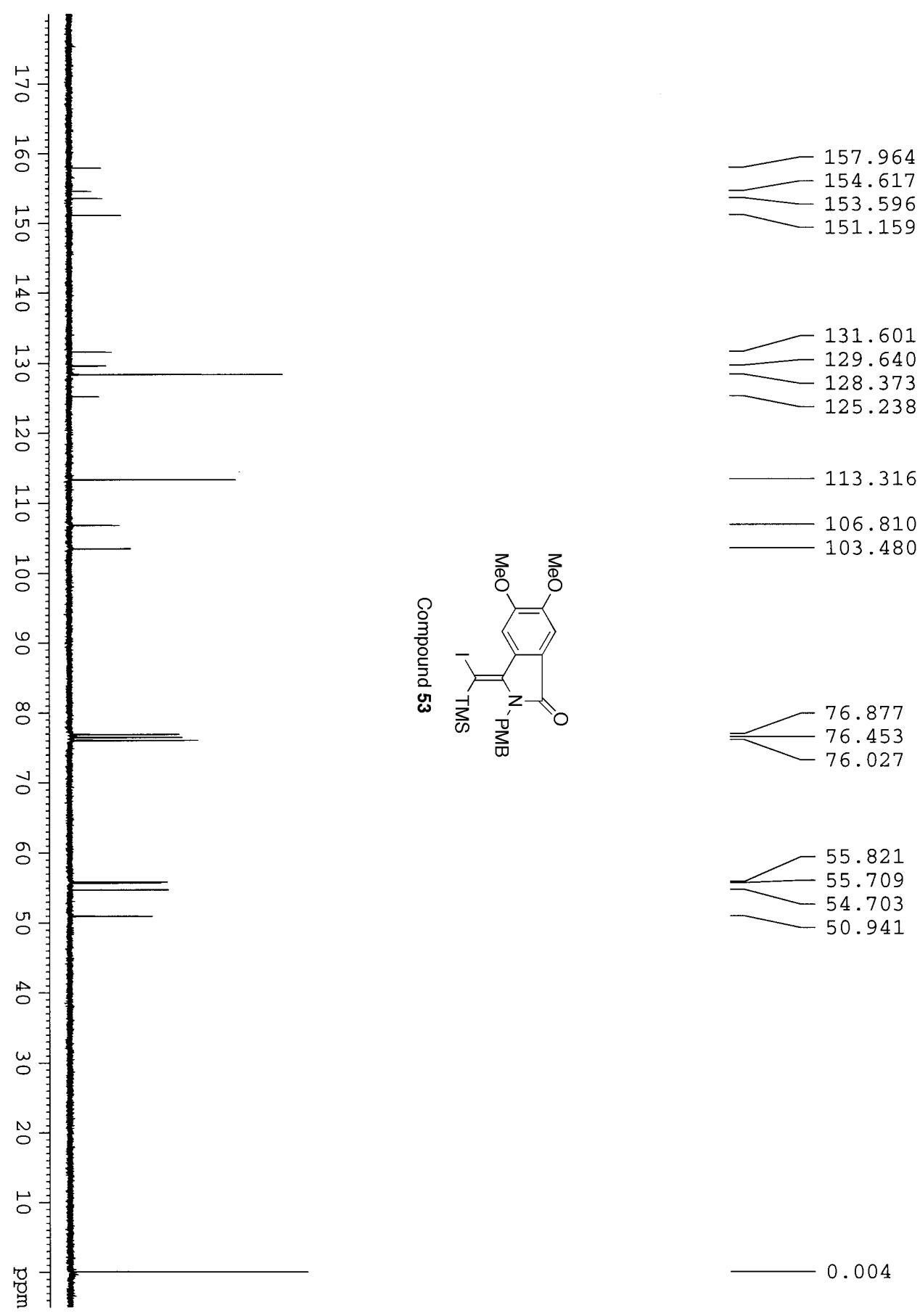

.004 


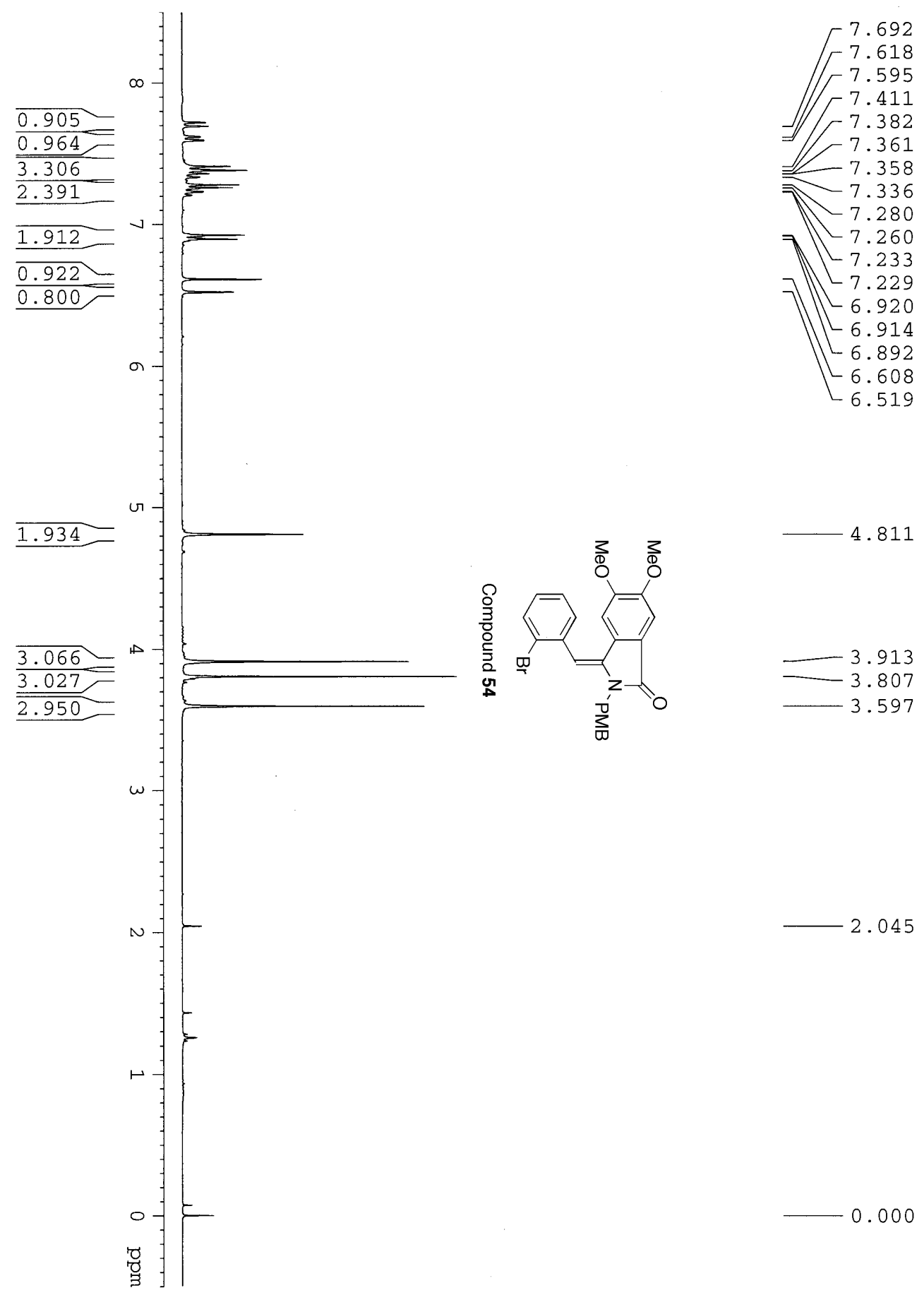



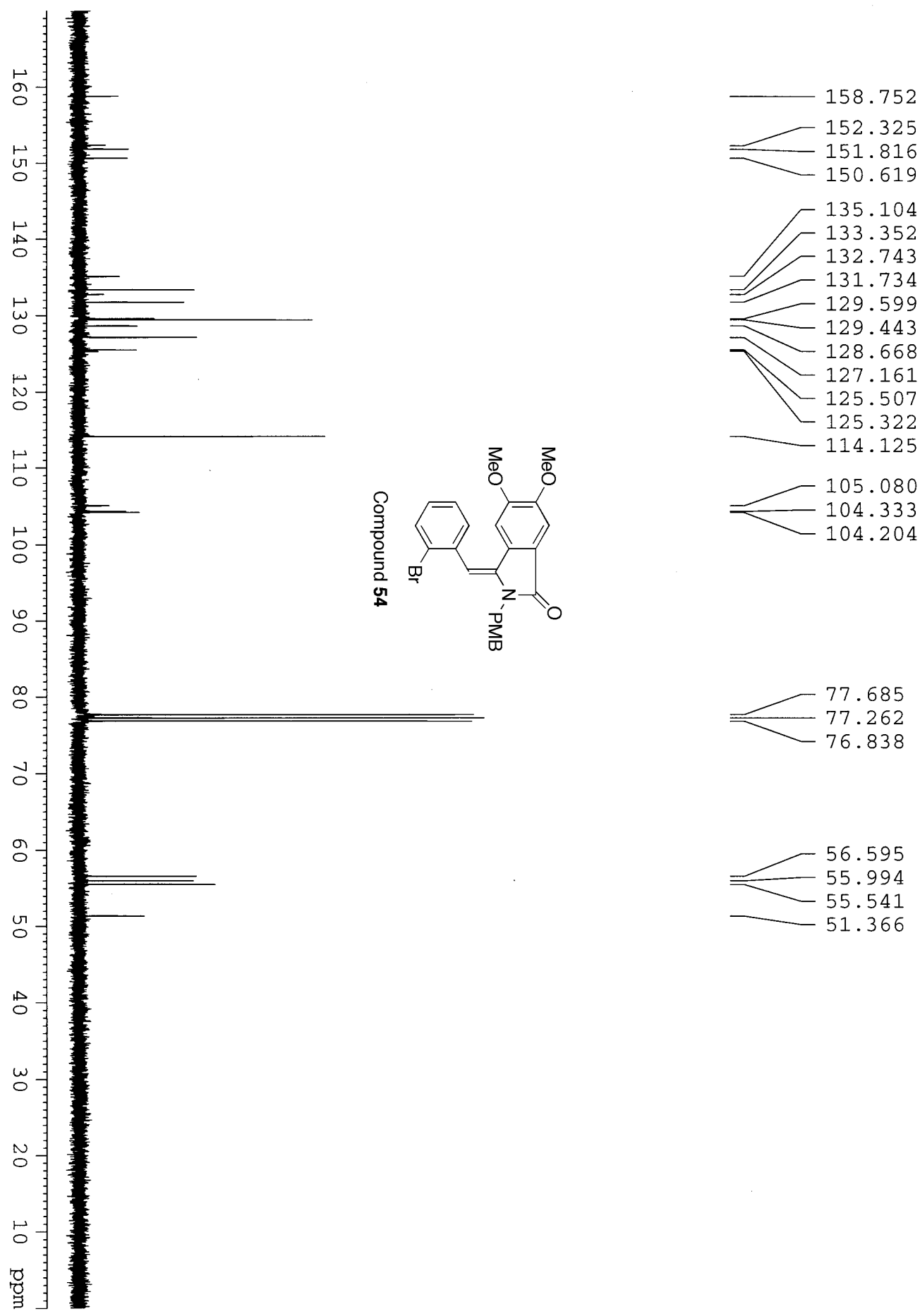